\title{
Estabilidade Assintótica Global e Continuação de Soluções Periódicas em Sistemas Suaves por Partes com Duas Zonas no Plano
}

Alexander Fernandes da Fonseca

\author{
Tese de Doutorado \\ Apresentada AO \\ Instituto De Matemática e Estatística \\ DA \\ Universidade de SÃo Paulo \\ PARA \\ OBTENÇÃO DO TÍTULO \\ $\mathrm{DE}$ \\ Doutor EM CiÊNCIAS
}

Programa: Matemática Aplicada

Orientador: Prof. Dr. Luis Fernando de Osório Mello 


\section{Estabilidade Assintótica Global e Continuação de Soluções Periódicas em Sistemas Suaves por Partes com Duas Zonas no Plano}

Esta versão da tese contém as correções e alterações sugeridas pela Comissão Julgadora durante a defesa da versão original do trabalho, realizada em 20/05/2016. Uma cópia da versão original está disponível no Instituto de Matemática e Estatística da Universidade de São Paulo.

Comissão Julgadora:

- Prof. Dr. Luis Fernando de Osório Mello (orientador) - UNIFEI

- Prof. Dr. Pedro Antonio Santoro Salomão - IME-USP

- Prof. Dr. Ricardo Miranda Martins - UNICAMP

- Prof. Dr. Maurício Firmino Silva Lima - UFABC

- Prof ${ }^{a}$. Dra . Ana Cristina de Oliveira Mereu - UFSCar-Sorocaba 


\section{Dedicatória}

Dedico esta tese a meu pai Sidney Fernandes da Fonseca (In memorian!1).

"Saudade é um sentimento que nos traz lembranças boas, mas dolorosas. É um sentimento do qual temos que aprender a lidar, pois há coisas na vida que não tem volta, e há coisas que simplesmente não estão sob nosso controle. A única certeza que temos é que o amor permanece o mesmo".

\footnotetext{
${ }^{1} 17 / 08 / 1953$ a $02 / 10 / 2008$.
} 


\section{Agradecimentos}

Agradeço primeiramente a minha família: Airidan (Mãe), Alessandra e Simone (Irmãs), Ithallo (Filho) e Thalles (Sobrinho) pelo reconhecimento de meu esforço em concluir o doutorado, um fato inédito na nossa família até então.

À Walquíria pelos conselhos, carinho e amor.

Peço desculpas a vocês pela ausência muitas vezes.

"Todas as vitórias ocultam uma abdicação". (Simone de Beauvoir)

Agradeço o Prof. Luis Fernando pela orientação e a confiança desde os tempos de mestrado. Aos professores Fábio e Baêta da UNIFEI pelos ensinamentos e orientações.

Aos colegas de trabalho e pesquisa do ICE...IMC-UNIFEI: Antonio, Bráulio, Denis e Lucas. Ao professor Sotomayor do IME pela ajuda na primeira parte do doutorado até a qualificação. Ao professor Clodoaldo e o IME pela oportunidade.

Aos professores João Luiz e Adilson pelo incentivo e orientação da graduação na UFOP. Aos companheiros de estudos na graduação no ICEB/UFOP: Éder, Júlio e Wenderson. Aos companheiros de estudos no mestrado na UNIFEI: Juliana, Larissa e Rafael. A todos que de forma direta ou indireta contribuiram para o cumprimento desta jornada.

"A persistência é o menor caminho do êxito". (Charles Chaplin)

"A liberdade é para a ciência o que o ar é para o animal". 


\section{Resumo}

Nesta tese estudamos um dos principais problemas na teoria qualitativa das equações diferenciais planares: o problema de determinar a bacia de atração de um ponto de equilíbrio. Damos uma prova rigorosa de que para sistemas lineares por partes de costura com duas zonas no plano, definidas por matrizes Hurwitz o único ponto de equilíbrio na reta de separação é globalmente assintoticamente estável. Por outro lado, provamos que nesta classe de sistemas, podemos ter um ponto de equilíbrio instável na origem quando uma curva poligonal separa as zonas, levando a um resultado contra-intuitivo do comportamento dinâmico de sistemas lineares por partes no plano. Além disso, estudamos os ciclos limites em perturbações suaves por partes de centros Hamiltonianos. Neste cenário, é comum adaptar resultados clássicos de sistemas suaves, como funções de Melnikov, para sistemas não-suaves. No entanto, existe pouca justificativa para este procedimento na literatura. Ao utilizar o método de regularização damos uma prova que suporta o uso de funções de Melnikov diretamente do problema não-suave original.

Palavras-chave: Sistema suave por partes, Método de Regularização, Ciclo limite, Função de Melnikov. 


\section{Abstract}

In this thesis we study one of the main problems in the qualitative theory of planar differential equations: the problem of determining the basin of attraction of an equilibrium point. We give a rigorous proof that for planar sewing piecewise linear systems with two zones, defined by Hurwitz matrices the unique equilibrium point in the separation straight line is globally asymptotically stable. On the other hand, we prove that sewing piecewise linear systems with two zones in the plane, defined by Hurwitz matrices can have one unstable equilibrium point at the origin allowing a broken line to separate the zones, leading to counter-intuitive dynamical behaviors of simple piecewise linear systems in the plane. Furthermore, we study limit cycles in piecewise smooth perturbations of Hamiltonians centers. In this setting it is common to adapt classical results for smooth systems, like Melnikov functions, to non-smooth ones. However, there is little justification for this procedure in the literature. By using the regularization method we give a proof that supports the use of Melnikov functions directly from the original non-smooth problem.

Keywords: Piecewise differential system, Regularization method, limit cycle, Melnikov function. 


\section{Conteúdo}

Resumo iv

Abstract vi

Sumário viii

1 Introducão

1.1 Motivacão . . . . . . . . . . . . . . . . . . . . 1

1.2 Definicão do problema . . . . . . . . . . . . . . . . . . 4

1.3 Estrutura do texto . . . . . . . . . . . . . . . . . . 5

2 Preliminares $\quad 7$

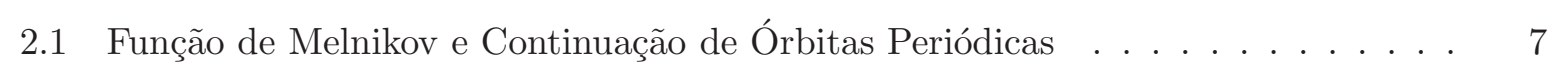

2.1.1 Perturbacão de um Centro Linear . . . . . . . . . . . . . . . . . 7

2.1.2 Perturbacão de um Sistema com Órbitas Periódicas . . . . . . . . . . . . 12

2.2 Regularizacão de Campos Descontínuos . . . . . . . . . . . . . . . . . . . . . 22

2.2 .1 Método de Regularizacão . . . . . . . . . . . . . . . . . . 22

2.2.2 Aplicação do Método de Regularização . . . . . . . . . . . . . . . . . . 25

\begin{tabular}{ll|r}
3 & Sistemas Lineares Hurwitzianos por Partes & 27
\end{tabular}

3.1 Estabilidade de Sistemas Lineares Hurwitzianos com Duas Zonas no Plano . . . 27

3.2 Coniunto de Separacão é uma Reta . . . . . . . . . . . . . . . . . . . . . 28

3.2.1 Caso 1. A origem é um nó atrator N1. . . . . . . . . . . . . . . 30

3.2 .2 Caso 2. A origem é um nó atrator N2. . . . . . . . . . . . . . . 32

3.2.3 Caso 3. A origem é um foco atrator FA. . . . . . . . . . . . . 33

3.3 Conjunto de Separação é uma Curva Poligonal . . . . . . . . . . . . . . . . . 36

4 Continuacão de Órbitas Periódicas e Regularizacão de Sistemas Descontínuos 43

4.1 Centro Linear Perturbado por Partes . . . . . . . . . . . . . . 43

4.2 Centro Hamiltoniano Descontínuo Perturbado por Partes . . . . . . . . . . 67 
4.3 Sistema Descontínuo com um Centro Perturbado por Partes . . . . . . . . . . 79

4.3.1 Extensões do Teorema de Diliberto e Lema Variacional . . . . . . . . . . 81

4.3.2 Continuação de Órbitas Periódicas . . . . . . . . . . . . . . . . . . 87

$\begin{array}{lll}5 & \text { Conclusões } & 93\end{array}$

$\begin{array}{ll}\text { Bibliografia } & 94\end{array}$ 


\section{Capítulo 1}

\section{Introdução}

Neste capítulo, são apresentadas a motivação da pesquisa e a definição do problema, bem como a estrutura do texto.

\subsection{Motivação}

Considere o campo vetorial $Z$ em $\mathbb{R}^{2}$ definido pelo sistema

$$
\dot{X}=Z(X)= \begin{cases}F^{-}(X), & \mathcal{H}(X) \leq 0 \\ F^{+}(X), & \mathcal{H}(X) \geq 0\end{cases}
$$

onde o ponto denota a derivada com respeito a variável independente $t$, chamada aqui de tempo, $X=(x, y) \in \mathbb{R}^{2}, F^{ \pm}$são campos vetoriais de classe $C^{k}, k \geq 1$, a função $\mathcal{H}: \mathbb{R}^{2} \rightarrow \mathbb{R}$, é pelo menos contínua e 0 é, geralmente, valor regular de $\mathcal{H}$. O conjunto $\Sigma=\mathcal{H}^{-1}(0)$, chamado de conjunto de separação, divide o plano em duas componentes (zonas) ilimitadas $\Sigma^{+}$e $\Sigma^{-}$, onde $\mathcal{H}$ é positiva e negativa, respectivamente. Assim $\mathbb{R}^{2}=\Sigma^{+} \cup \Sigma \cup \Sigma^{-}$.

Chamamos (1.1) de sistema suave por partes com duas zonas no plano e o campo $Z$ definido por este sistema de campo vetorial descontínuo no plano. Muitos sistemas dinâmicos ocorrem naturalmente na descrição física de processos suaves por partes, isto é, o seu movimento é caracterizado por períodos de evolução suaves que são interrompidos por um evento instantâneo. A dinâmica descontínua do sistema (1.1) pode ser observada em várias situações, como em sistemas mecânicos com impactos, teoria de controle, eletrônica e biologia. Ver por exemplo [9], 12], 21], 22.

Um livro clássico que trata do estudo dos sistemas suaves por partes no plano (Sistemas de Filippov) é o livro de Filippov [14]. Este livro estabelece uma teoria em torno do problema descontínuo e desenvolve, entre outras coisas, as chamadas convenções de Filippov, que tratam de dizer como pode ocorrer a transição das órbitas entre as regiões $\Sigma^{+}$e $\Sigma^{-}$, ou, se for o caso, 
de como as órbitas podem permanecer sobre $\Sigma$. Filippov, neste trabalho, introduziu o que vem a ser em $\Sigma$ os pontos de costura, escape, deslize ou pontos de tangência.

Consideremos um sistema linear homogêneo no plano na forma

$$
\dot{X}=A X
$$

onde $A$ é uma matriz real de ordem $2 \times 2$. Da teoria clássica de sistemas de equações diferenciais ordinárias, se os autovalores da matriz $A$ têm partes reais negativas, então o único equilíbrio na origem é globalmente assintoticamente estável. Uma questão que surge é dada no problema abaixo.

Problema 1.1.1. Podemos estender o resultado sobre estabilidade global do sistema (1.2) para a classe de sistemas lineares homogêneos suaves por partes?

Sob algumas hipóteses, uma resposta ao Problema 1.1.1 é dada a seguir. Suponhamos que o campo $Z$ dado em (1.1) seja linear. Em geral, sistemas de equações diferenciais ordinárias lineares por partes com duas zonas no plano são definidos por

$$
\dot{X}= \begin{cases}A^{-} X+B^{-}, & \mathcal{H}(X) \leq 0, \\ A^{+} X+B^{+}, & \mathcal{H}(X) \geq 0,\end{cases}
$$

onde $A^{ \pm}$são matrizes reais $2 \times 2, B^{ \pm}$são matrizes reais $2 \times 1$.

Desde o trabalho seminal de Andronov et al. [2], vários artigos foram publicados, principalmente sobre questões como a existência, número, a estabilidade e a distribuição de ciclos limites para a classe de sistemas dado em (1.3). Veja [6], [7, [8], [16], [25], [26], [27], [28], [33] e as referências nestes artigos. Estes estudos foram desenvolvidos tendo em conta aspectos como o número e estabilidade de pontos de equilíbrio, assim como a sua posição com respeito ao conjunto de separação $\Sigma$ e o comportamento dos campos de vetores lineares em $\Sigma$. No entanto, há poucos artigos sobre o problema da estabilidade global de um ponto de equilíbrio nesta classe de sistemas (veja [19] para um estudo e aplicação na teoria de controle). O marco de tal estudo é o artigo [15] em que os autores analisaram o caso em que:

H1. $A^{+}$e $A^{-}$são matrizes Hurwitzianas (a parte real de todos os autovalores é negativa);

H2. $B^{+}=B^{-}=0$;

H3. o conjunto de separação $\Sigma$ é uma reta que contém o único ponto de equilíbrio do sistema na origem;

H4. os campos de vetores $A^{+} X$ e $A^{-} X$ são contínuos em $\Sigma-\{(0,0)\}$. 
Com as hipóteses acima, em [15] provou-se que o único ponto de equilíbrio é globalmente assintoticamente estável.

Agora, considere o sistema suave no plano

$$
\dot{X}=F(X)+\varepsilon G(X)
$$

onde $F, G$ são campos de vetores suaves no plano e $\varepsilon \geq 0$ é um parâmetro real.

Suponha que para $\varepsilon=0$ o sistema (1.4) tem um anel $\mathcal{A}$ de órbitas periódicas. Uma pergunta pode ser colocada:

Problema 1.1.2. Quantos ciclos limites bifurcam do anel $\mathcal{A}$ de órbitas periódicas quando $\varepsilon$ é suficientemente pequeno?

Existem duas ferramentas clássicas que podem responder a esta questão: Funções de PoincaréAndronov-Melnikov e a Teoria da Média (Averaging). A primeira chamaremos simplesmente de Funções de Melnikov. Se $a \mapsto \mathcal{M}(a)$ é a função de Melnikov para o sistema (1.4), o número de zeros simples positivos desta função é o número de órbitas periódicas persistentes (ciclos limites). Ver [11].

Agora, considere uma $\varepsilon$-perturbação autonôma descontínua (em geral) do campo $Z$ dado em (1.1) definida pelo sistema

$$
\dot{X}=Z(X, \varepsilon)= \begin{cases}F^{-}(X)+\varepsilon G^{-}(X), & \mathcal{H}(X) \leq 0, \\ F^{+}(X)+\varepsilon G^{+}(X), & \mathcal{H}(X) \geq 0,\end{cases}
$$

onde $G^{ \pm}$são campos vetoriais de classe $C^{k}, k \geq 1$ e $\varepsilon \geq 0$ é um parâmetro real. Suponhamos que para $\varepsilon=0$ o sistema (1.5) tem um anel $\mathcal{A}$ de órbitas periódicas, onde estas órbitas periódicas serão dadas em geral por curvas suaves por partes. Como no caso suave, as duas ferramentas que podem responder o Problema 1.1.2 no contexto dos sistemas suaves por partes no plano são as Funções de Melnikov e a Teoria da Média (Averaging). Para o primeiro caso ver [24, 39, 40] e para o segundo veja [29, 32] e suas referências.

No que diz respeito ao problema das funções de Melnikov acima ou da teoria da média, estes foram utilizados diretamente a partir do problema não suave dado pelo sistema (1.5). Sotomayor e Teixeira introduziram o método de regularização de campos descontínuos em [38], cuja ideia principal é tornar o sistema descontínuo em um sistema suave (sistema regularizado), onde a teoria clássica poderá ser aplicada. Para mais detalhes e aplicações deste método ver por exemplo [30], 31], 37] ou [38. 


\subsection{Definição do problema}

Nesta tese estudaremos somente o caso em que os pontos sobre $\Sigma$ são pontos de costura, isto é, para os sistemas suaves por partes (1.1),$X_{0} \in \Sigma$ é ponto de costura se

$$
\left(F^{-}\left(X_{0}\right) \cdot \nabla \mathcal{H}\left(X_{0}\right)\right)\left(F^{+}\left(X_{0}\right) \cdot \nabla \mathcal{H}\left(X_{0}\right)\right)>0
$$

Inicialmente, como uma resposta ao Problema 1.1.1, vamos estender o resultado sobre a estabilidade assintótica global mudando a hipótese $\mathbf{H} 4$ à seguinte

H4'. Os pontos em $\Sigma-\{(0,0)\}$ são do tipo costura.

Mais precisamente, provamos o seguinte teorema.

Teorema 1.2.1. Considere o sistema (1.3) com as hipóteses $\mathbf{H 1}, \mathbf{H 2}, \mathbf{H 3}$ and H4'. Então o único ponto de equilíbrio que é a origem é globalmente assintoticamente estável.

É apropriado observar que as hipóteses do Teorema 1.2.1 asseguram que o sistema (1.3) é um sistema planar bimodal bem posto na terminologia da teoria de controle (ver [19]). Além disso, a curva de separação $\Sigma$ entre as duas zonas desempenha um papel importante nos sistemas diferenciais lineares planares seccionalmente descontínuos. No artigo [7] foi exibido um exemplo de tal sistema com sete ciclos limites tendo $\Sigma$ como uma curva poligonal. No artigo [8] os autores comprovaram a existência de uma classe de sistemas diferenciais lineares por partes com duas zonas no plano separados por uma curva poligonal $\Sigma$ tendo exatamente $n$ ciclos limites hiperbólicos, para um dado inteiro positivo $n$.

Consideremos a hipótese:

H3'. o conjunto de separação $\Sigma$ é uma curva poligonal dada pela união de duas semi-retas que contêm um único ponto de equilíbrio na origem do sistema.

Provamos também, o seguinte teorema.

Teorema 1.2.2. Considere o sistema (1.3) com as hipóteses H1, H2, H3' and H4'. Existem matrizes Hurwitzianas $A^{+}$e $A^{-}$tal que o único ponto de equilíbrio na origem é ou um foco estável, ou um centro, ou um foco instável.

Este teorema está relacionado com o artigo [10], onde os autores provaram que os sistemas lineares por partes contínuos em $\mathbb{R}^{3}$ com duas zonas separadas por um plano e definidas por matrizes Hurwitz podem ter um ponto de equilíbrio instável na origem.

Como dissemos, uma resposta ao Problema 1.1.2 pode ser dada pelas funções de Melnikov. Considere os seguintes casos para o sistema (1.5): 
(a) $F^{ \pm}(X)=(-y, x)$ e $G^{ \pm}(X)=\left(g_{1}^{ \pm}(x, y), g_{2}^{ \pm}(x, y)\right)$,

(b) $\left.F^{ \pm}(X)=\left(-H_{y}(x, y)\right), H_{x}(x, y)\right)$ e $G^{ \pm}(X)=\left(g_{1}^{ \pm}(x, y), g_{2}^{ \pm}(x, y)\right)$,

(c) $F^{ \pm}(X)=\left(-H_{y}^{ \pm}(x, y), H_{x}^{ \pm}(x, y)\right)$ e $G^{ \pm}(X)=\left(g_{1}(x, y), g_{2}(x, y)\right)$,

(d) $F^{ \pm}(X)=\left(-H_{y}^{ \pm}(x, y), H_{x}^{ \pm}(x, y)\right)$ e $G^{ \pm}(X)=\left(g_{1}^{ \pm}(x, y), g_{2}^{ \pm}(x, y)\right)$,

(e) $F^{ \pm}(X)=\left(f_{1}(x, y), f_{2}(x, y)\right)$ e $G^{ \pm}(X)=\left(g_{1}^{ \pm}(x, y), g_{2}^{ \pm}(x, y)\right)$,

(f) $F^{ \pm}(X)=\left(f_{1}^{ \pm}(x, y), f_{2}^{ \pm}(x, y)\right)$ e $G^{ \pm}(X)=\left(g_{1}(x, y), g_{2}(x, y)\right)$,

(g) $F^{ \pm}(X)=\left(f_{1}^{ \pm}(x, y), f_{2}^{ \pm}(x, y)\right)$ e $G^{ \pm}(X)=\left(g_{1}^{ \pm}(x, y), g_{2}^{ \pm}(x, y)\right)$.

Em cada caso acima encontramos uma função de Melnikov associada, com especial interesse nos casos (a), (b), (c) e (d). Ver [24].

Nos casos onde temos um centro linear (item (a)) ou um centro Hamiltoniano (item (b)) usamos o método de regularização e damos uma prova para o uso de funções de Melnikov diretamente no problema não-suave original. Na verdade, estudamos a equivalência de ambos os métodos nestes dois casos. Denotemos a função de Melnikov do sistema regularizado por $\mathcal{R}(a, \mu)$, onde $a \in \mathbb{R}$ e $\mu>0$ é chamado parâmetro de regularização e a função de Melnikov do sistema não regularizado por $\mathcal{M}(a)$. Para os itens (a) e (b) provamos o seguinte teorema.

Teorema 1.2.3. As funções $\mathcal{R}(a, \mu)$ e $\mathcal{M}(a)$ satisfazem para cada a $>0$ a seguinte relação

$$
\lim _{\mu \rightarrow 0} \mathcal{R}(a, \mu)=\mathcal{M}(a)
$$

\subsection{Estrutura do texto}

Este trabalho está dividido como segue. No capítulo 2 obtemos a função de Melnikov para sistemas suaves, além disso, fazemos uma introdução do método de regularização de campos descontínuos e das convenções de Filippov. No capítulo 3 demonstramos os Teoremas 1.2.1 e 1.2.2. O capítulo 4 trata de estabelecer uma forma para a função de Melnikov no caso dos sistemas suaves por partes e demonstrar a relação dada no Teorema 1.2.3, além disso apresentamos alguns exemplos. No capítulo 5 fazemos algumas considerações finais e propostas de trabalhos futuros. 


\section{Capítulo 2}

\section{Preliminares}

Neste capítulo apresentamos a função de Melnikov de primeira ordem para um sistema suave no plano perturbado, o método de regularização de campos descontínuos no plano e as convenções de Filippov. As principais referências deste capítulo são [11, [14] e [38. Este capítulo visa dar um suporte técnico mínimo para os outros capítulos, entretanto alguns tópicos clássicos da Análise podem surgir naturalmente ao longo da tese, entre os quais destacamos: Teorema da Função Implícita, dependência diferenciável e contínua das soluções com respeito aos parâmetros e condições iniciais e o Teorema da existência e unicidade de soluções de EDO. Não enunciaremos tais resultados, e sempre que acharmos necessário faremos menção a tais resultados. Sugerimos como leitura complementar os livros [18], 35] e [36].

\subsection{Função de Melnikov e Continuação de Órbitas Periódicas}

Nesta seção vamos obter a função de Melnikov, que permitirá dizer quantos ciclos limites bifurcam de um anel $\mathcal{A}$ de órbitas periódicas de um sistema suave no plano quando o parâmetro de perturbação é suficientemente pequeno. Muitos dos conceitos e demonstrações nesta seção serão utilizados no Capítulo 4. Começamos com o problema mais simples de perturbação de um centro linear e estendemos para o caso mais geral.

\subsubsection{Perturbação de um Centro Linear}

Considere o sistema

$$
\dot{X}=A X+\varepsilon G(X),
$$


com $X=(x, y) \in \mathbb{R}^{2}, \varepsilon$ um parâmetro real, o ponto denotando a derivada com respeito ao parâmetro $t$,

$$
A=\left(\begin{array}{cc}
0 & -1 \\
1 & 0
\end{array}\right),
$$

e $G(x, y)=\left(g_{1}(x, y), g_{2}(x, y)\right)$ campo vetorial de classe $C^{k}, k \geq 1$ e $G(0,0)=(0,0)$. O sistema (2.1) é uma perturbação do sistema

$$
\dot{X}=A X
$$

Se $\varepsilon=0$ em (2.1), então todas as órbitas do sistema (2.1) (do sistema (2.2)), exceto para a origem, são circunferências, isto é, a origem é um centro linear. Diremos que o sistema (2.1) é o sistema perturbado e o sistema (2.2) o sistema não perturbado. Agora, para $\varepsilon \neq 0$ e $a>0$ seja $(t, a, \varepsilon) \mapsto X(t, a, \varepsilon)=(x(t, a, \varepsilon), y(t, a, \varepsilon))$ solução do sistema (2.1) que satisfaz a condição inicial

$$
X(0, a, \varepsilon)=(x(0, a, \varepsilon), y(0, a, \varepsilon))=(a, 0) .
$$

Segue da dependência contínua com respeito aos parâmetros e condições iniciais que esta solução, para $\varepsilon \neq 0$ suficientemente pequeno, permanece próxima ao círculo de raio $a$ pelo menos até o retorno ao eixo- $x$ após um tempo finito positivo $T(a, \varepsilon)$.

Resulta que:

1. $y(T(a, \varepsilon), a, \varepsilon)=0$,

2. $T(a, 0)=2 \pi$,

3. $x(2 \pi, a, 0)=a$.

Defina os seguintes conjuntos

$$
L=\left\{(x, 0) \in \mathbb{R}^{2}: x>0\right\}, \quad I_{\varepsilon_{0}}=\left(-\varepsilon_{0}, \varepsilon_{0}\right), \varepsilon_{0}>0 .
$$

Sejam as seguintes famílias a um parâmetro $\varepsilon$ de aplicações de Poincaré parametrizadas

$$
\begin{aligned}
\Pi^{\varepsilon}: L & \longrightarrow L \\
& a \longmapsto \Pi^{\varepsilon}(a)=\Pi(a, \varepsilon)=x(T(a, \varepsilon), a, \varepsilon)
\end{aligned}
$$

A família de funções do tipo separação é dada por

$$
\begin{aligned}
\delta: L \times I_{\varepsilon_{0}} & \longrightarrow \mathbb{R} \\
(a, \varepsilon) & \longmapsto \delta(a, \varepsilon)=\Pi(a, \varepsilon)-a .
\end{aligned}
$$

Se $\Pi(a, \varepsilon)=a$, então $X(t, a, \varepsilon)=(x(t, a, \varepsilon), y(t, a, \varepsilon))$ é uma solução periódica do sistema (2.1) com período $T(a, \varepsilon)$, isto é, $a$ é ponto fixo da aplicação $\Pi$ (zero da função $\delta$ ) e o ponto $(a, 0)$ é o ponto inicial para uma órbita periódica do sistema perturbado (2.1).

Resulta que: 
1. $\delta(a, 0) \equiv 0$, pois

$$
\begin{aligned}
\delta(a, 0) & =\Pi(a, 0)-a \\
& =x(T(a, 0), a, 0)-a \\
& =x(2 \pi, a, 0)-a=0, \forall a \in L ;
\end{aligned}
$$

2. Tomando as expansão de Taylor da função $\delta$, com respeito à variável $\varepsilon \in I_{\varepsilon_{0}}$ em torno de $\varepsilon=0$ obtemos

$$
\delta(a, \varepsilon)=\varepsilon \delta_{\varepsilon}(a, 0)+O\left(a, \varepsilon^{2}\right)=\varepsilon\left(\delta_{\varepsilon}(a, 0)+O(a, \varepsilon)\right)
$$

sendo

$$
\delta_{\varepsilon}(a, 0)=\left.\frac{\partial}{\partial \varepsilon} \delta(a, \varepsilon)\right|_{\varepsilon=0} .
$$

Defina a função

$$
\Delta(a, \varepsilon):=\delta_{\varepsilon}(a, 0)+O(a, \varepsilon) .
$$

Veja que

$\delta_{a}(a, 0) \equiv 0, \forall a \in L$, pois

$$
\left.\Pi_{a}(a, \varepsilon)\right|_{\varepsilon=0}=\left.\left(1+\varepsilon \Delta_{a}(a, \varepsilon)\right)\right|_{\varepsilon=0}=1,
$$

sendo

$$
\delta_{a}(a, 0)=\left.\frac{\partial}{\partial a} \delta(a, \varepsilon)\right|_{\varepsilon=0}, \quad \Pi_{a}(a, \varepsilon)=\frac{\partial}{\partial a} \Pi(a, \varepsilon) \quad \text { e } \quad \Delta_{a}(a, \varepsilon)=\frac{\partial}{\partial a} \Delta(a, \varepsilon) .
$$

Em virtude disso, não podemos aplicar o Teorema da Função Implícita diretamente. Porém, se $a_{0}$ é zero simples da função $\Delta$ dada em (2.3) calculada em $\varepsilon=0$, isto é,

(i) $\Delta\left(a_{0}, 0\right)=0,\left(\delta_{\varepsilon}\left(a_{0}, 0\right)=0\right)$;

(ii) $\Delta_{a}\left(a_{0}, 0\right) \neq 0,\left(\delta_{a \varepsilon}\left(a_{0}, 0\right) \neq 0\right)$,

pelo Teorema da Função Implícita, existe um intervalo $I \subset I_{\varepsilon_{0}}$ e uma única função

$$
\begin{aligned}
\phi: I \longrightarrow L \\
\varepsilon \longmapsto a=\phi(\varepsilon)
\end{aligned}
$$

tal que

(a) $\phi(0)=a_{0}$;

(b) $\Delta(\phi(\varepsilon), \varepsilon)=0$ para todo $\varepsilon \in I$;

(c) $a=a_{0}-\frac{\Delta_{\varepsilon}\left(a_{0}, 0\right)}{\Delta_{a}\left(a_{0}, 0\right)} \varepsilon+O\left(\varepsilon^{2}\right)$. 
Como $\delta(a, \varepsilon)=\varepsilon \Delta(a, \varepsilon)$ e $a=\phi(\varepsilon)$, então

$$
\delta_{\varepsilon}(a, \varepsilon)=\Delta(a, \varepsilon)+\varepsilon\left(\Delta_{a}(a, \varepsilon) \phi^{\prime}(\varepsilon)+\Delta_{\varepsilon}(a, \varepsilon)\right) .
$$

Logo, em $\varepsilon=0$ temos

$$
\delta_{\varepsilon}\left(a_{0}, 0\right)=\Delta\left(a_{0}, 0\right)
$$

Portanto, a busca de uma solução periódica do sistema perturbado (2.1) consiste em encontrar soluções implícitas da equação $\Delta(a, 0)=0$. Obtemos o seguinte teorema.

Teorema 2.1.1. Se a $>0$ é um zero simples da função $\Delta$ dada em (2.3), isto é, $\Delta(a, 0)=0$ e $\Delta_{a}(a, 0) \neq 0$, ou equivalentemente se $\delta_{\varepsilon}(a, 0)=0$ e $\delta_{a \varepsilon}(a, 0) \neq 0$, então a solução do sistema perturbado (2.1) com condição inicial no ponto $(a, 0)$ é uma solução periódica (ciclo limite).

Portanto, basta estudar os zeros simples da função $\delta_{\varepsilon}(a, 0)$, pois, para todo $\varepsilon \in I$ obtemos $\Delta(\phi(\varepsilon), \varepsilon)=0$, ou seja, $\delta(\phi(\varepsilon), \varepsilon)=0$.

Definição 2.1.1. A função $\delta_{\varepsilon}(a, 0)$ é chamada de função de separação reduzida, e um zero simples desta função (respectivamente a correspondente órbita periódica não perturbada) é chamado de ponto de continuação das soluções periódicas do sistema (2.1) (respectivamente órbita periódica continuável). Além disso, uma órbita periódica é chamada de persistente se esta for continuável.

A estabilidade de uma solução periódica do sistema perturbado (2.1) passando próximo a um ponto de continuação $(a, 0)$ é determinado por $\Pi_{a}(a, \varepsilon)$ : Se $\Pi_{a}(a, \varepsilon)<1$, então a solução periódica é (assintoticamente) estável; e se $\Pi_{a}(a, \varepsilon)>1$, então a solução periódica é instável. Veja que

$$
\left.\Pi_{a}(a, \varepsilon)\right|_{a=\phi(\varepsilon)}=1+\varepsilon \Delta_{a}(\phi(\varepsilon), \varepsilon)=1+\varepsilon \delta_{a \varepsilon}\left(a_{0}, 0\right)+O\left(\varepsilon^{2}\right),
$$

$\operatorname{logo}$, para $\varepsilon \neq 0$ suficientemente pequeno a estabilidade da solução periódica é dada por $\delta_{a \varepsilon}\left(a_{0}, 0\right)$, se por exemplo, $\varepsilon>0$ é suficientemente pequeno e $\delta_{a \varepsilon}\left(a_{0}, 0\right)<0$, então a órbita periódica é estável.

Teorema 2.1.2. Uma solução periódica $X(t, a, \varepsilon)$ do sistema (2.1) para $\varepsilon \neq 0$ suficientemente pequeno é estável se $\varepsilon$ e $\delta_{a \varepsilon}\left(a_{0}, 0\right)$ têm sinais opostos, caso contrário é instável.

Observação 2.1.1. Uma forma equivalente para os Teoremas [2.1.1] e 2.1.2, com $\varepsilon \geq 0$ é a seguinte: Se $a_{0}>0$ é um zero simples da função $\delta_{\varepsilon}(a, 0)$, então para $\varepsilon>0$ suficientemente pequeno existe um ciclo limite $X^{\varepsilon}$ de (2.1) tal que $X^{\varepsilon}$ tende ao círculo de centro na origem e raio $a_{0}$ quando $\varepsilon$ tende a 0 . O ciclo limite é estável se $\delta_{a \varepsilon}\left(a_{0}, 0\right)<0$ e instável se $\delta_{a \varepsilon}\left(a_{0}, 0\right)>0$. 
Suponhamos daqui por diante que $\varepsilon \geq 0$ no sistema (2.1). Precisamos obter uma expressão para a função $\delta_{\varepsilon}(a, 0)$ em termos das funções do sistema (2.1).

Temos que $\delta(a, \varepsilon)=x(T(a, \varepsilon), a, \varepsilon)-a$, derivando $\delta$ com respeito a $\varepsilon$ e avaliando em $(a, 0)$ obtemos

$$
\delta_{\varepsilon}(a, 0)=\dot{x}(T(a, 0), a, 0) T_{\varepsilon}(a, 0)+x_{\varepsilon}(T(a, 0), a, 0) .
$$

Como $\dot{x}(T(a, 0), a, 0)=-y(2 \pi, a, 0)=0$,

$$
\delta_{\varepsilon}(a, 0)=x_{\varepsilon}(T(a, 0), a, 0)=x_{\varepsilon}(2 \pi, a, 0) .
$$

Segue da dependência diferenciável das soluções com respeito aos parâmetros e condições iniciais que uma solução de (2.1) é diferenciável com respeito ao parâmetro $\varepsilon$. Assim, para obter a expressão de $x_{\varepsilon}(2 \pi, a, 0)$ na equação (2.4) basta derivar ambos os membros do sistema (2.1) e a condição inicial com respeito a $\varepsilon$ para $\varepsilon=0$, obtendo o seguinte problema de valor inicial variacional

$$
\left\{\begin{array}{c}
\dot{X}_{\varepsilon}=A X_{\varepsilon}+G(X), \\
X_{\varepsilon}(0, a, 0)=(0,0) .
\end{array}\right.
$$

Uma solução de (2.5) é contínua e tem a forma $X_{\varepsilon}(t, a, 0)=\left(x_{\varepsilon}(t, a, 0), y_{\varepsilon}(t, a, 0)\right)$ e pode ser obtida pelo Método da Variação de Parâmetros. O sistema homogêneo associado dado por $\dot{X}_{\varepsilon}=A X_{\varepsilon}$ tem como matriz fundamental

$$
\Phi(t)=\left(\begin{array}{cc}
\cos (t) & -\operatorname{sen}(t) \\
\operatorname{sen}(t) & \cos (t)
\end{array}\right),
$$

que satisfaz $\dot{\Phi}=A \Phi$ e $\Phi(0)=I$, onde $I$ é a matriz identidade de ordem 2. Logo, a solução de (2.5) é dada por

$$
X_{\varepsilon}(t, a, 0)=\Phi(t) X_{\varepsilon}(0, a, 0)+\Phi(t) \int_{0}^{t} \Phi^{-1}(s) G\left(\gamma_{a}(s)\right) \mathrm{d} s,
$$

onde $\gamma_{a}(s)=(a \cos (s), a \operatorname{sen}(s))$. Como $X_{\varepsilon}(0, a, 0)=(0,0)$ e $T(a, 0)=2 \pi$, obtemos que

$$
X_{\varepsilon}(2 \pi, a, 0)=\Phi(2 \pi) \int_{0}^{2 \pi} \Phi^{-1}(s) G\left(\gamma_{a}(s)\right) \mathrm{d} s .
$$

Temos que $\Phi(2 \pi)=I$ e

$$
\Phi^{-1}(t)=\left(\begin{array}{cc}
\cos (t) & \operatorname{sen}(t) \\
-\operatorname{sen}(t) & \cos (t)
\end{array}\right)
$$

logo,

$$
I \cdot \Phi^{-1}(s) \cdot G\left(\gamma_{a}(s)\right)=\left(g_{1}\left(\gamma_{a}(s)\right) \cos (s)+g_{2}\left(\gamma_{a}(s)\right) \operatorname{sen}(s),-g_{1}\left(\gamma_{a}(s)\right) \operatorname{sen}(s)+g_{2}\left(\gamma_{a}(s)\right) \cos (s)\right) .
$$

Portanto, de (2.6) obtemos

$$
x_{\varepsilon}(2 \pi, a, 0)=\int_{0}^{2 \pi} g_{1}(a \cos (s), a \operatorname{sen}(s)) \cos (s)+g_{2}(a \cos (s), a \operatorname{sen}(s)) \operatorname{sen}(s) \mathrm{d} s .
$$


Teorema 2.1.3. Se a $>0$ é um zero simples da função

$$
a \mapsto \mathcal{M}(a)=\int_{0}^{2 \pi} g_{1}(a \cos (s), a \operatorname{sen}(s)) \cos (s)+g_{2}(a \cos (s), a \operatorname{sen}(s)) \operatorname{sen}(s) \mathrm{d} s,
$$

então para $\varepsilon>0$ suficientemente pequeno existe um ciclo limite $X^{\varepsilon}$ de (2.1) tal que $X^{\varepsilon}$ tende ao círculo de centro na origem e raio a quando e tende a 0 . O ciclo limite é estável se $\mathcal{M}^{\prime}(a)<0$ e instável se $\mathcal{M}^{\prime}(a)>0$.

\subsubsection{Perturbação de um Sistema com Órbitas Periódicas}

Agora, considere o caso geral dado pelo sistema

$$
\dot{X}=F(X)+\varepsilon G(X)
$$

como antes, $X=(x, y) \in \mathbb{R}^{2}, \varepsilon$ um parâmetro real e o ponto denotando a derivada com respeito ao parâmetro $t$. Além disso, $F(x, y)=\left(f_{1}(x, y), f_{2}(x, y)\right)$ e $G(x, y)=\left(g_{1}(x, y), g_{2}(x, y)\right)$ são campos vetoriais de classe $C^{k}, k \geq 1$. Suponha que o sistema não perturbado dado por

$$
\dot{X}=F(X)
$$

tem soluções periódicas. Se o sistema (2.8) é não linear temos dois casos a considerar:

1. Existe um ciclo limite;

2. Existe uma região anular invariante de soluções periódicas.

No caso do ciclo limite, podemos determinar se o ciclo limite persiste após a perturbação; e no caso da região anular invariante de soluções periódicas, podemos determinar qual, se alguma, destas soluções periódicas persiste.

Suponhamos em geral que o sistema não perturbado (2.8) tem uma solução periódica $\Gamma$ e sem perda de generalidade, suponhamos que $\Gamma$ tenha orientação horária. Escolha um ponto $X_{0} \in \Gamma$ e uma curva $C$ que é transversal à $\Gamma$ em $X_{0}$. Para aplicação do Teorema da Função Implícita, deve existir um subconjunto aberto $C_{0} \subseteq C$ com $X_{0} \in C$ e algum $\varepsilon_{0}>0$ tal que para cada $\sigma \in C_{0}$ a solução do sistema perturbado (2.7) $\mathrm{com}|\varepsilon|<\varepsilon_{0}$ retorna a um ponto $\widetilde{\sigma}$ em $C$ após algum tempo finito positivo. Logo, existe uma função tempo de retorno

$$
\begin{aligned}
\mathcal{T}: C_{0} \times I_{\varepsilon_{0}} & \longrightarrow \mathbb{R} \\
(\sigma, \varepsilon) & \longmapsto \tau(\sigma, \varepsilon)
\end{aligned}
$$

e uma aplicação de Poincaré parametrizada

$$
\begin{aligned}
\Pi: C_{0} \times I_{\varepsilon_{0}} & \longrightarrow C \\
(\sigma, \varepsilon) & \longmapsto \Pi(\sigma, \varepsilon) .
\end{aligned}
$$


Chamamos o subconjunto $C_{0} \subseteq C$ de Seção de Poincaré.

Tome uma função

$$
\begin{gathered}
\sigma: \mathbb{R} \longrightarrow C_{0} \subseteq \mathbb{R}^{2} \\
a \longmapsto \sigma(a)
\end{gathered}
$$

tal que $\sigma(0)=X_{0}$ e para cada $a \in \mathbb{R}$ o vetor $\dot{\sigma}(a)$ é um vetor não nulo tangente à $C_{0}$ em $\sigma(a)$. Definimos a função deslocamento a partir de (2.10) por

$$
\delta(a, \varepsilon):=\sigma^{-1}(\Pi(\sigma(a), \varepsilon))-a .
$$

Dessa forma estamos identificando a curva $C$ como uma variedade unidimensional e definindo $\delta$ relativa a uma representação local de coordenadas de $C_{0}$.

Vamos supor primeiramente que $\Gamma$ é um ciclo limite para o sistema não perturbado (2.8) com período $2 \pi / \omega$. Nosso objetivo é dizer algo sobre a estabilidade de $\Gamma$ e estabelecer resultados necessários para obtenção da função de Melnikov.

Temos que $\delta(0,0)=0$, pois

$$
\sigma^{-1}(\Pi(\sigma(0), 0))=\sigma^{-1}\left(\Pi\left(X_{0}, 0\right)\right)=\sigma^{-1}\left(X_{0}\right)=0 .
$$

Como $\Gamma$ é ciclo limite ele é uma solução periódica isolada, então a função $a \mapsto \delta_{a}(a, 0)$ é não nula numa vizinhança de $a=0$, portanto o Teorema da Função Implícita pode ser aplicado diretamente. Portanto, pelo Teorema da Função Implícita existe uma única função contínua $\varepsilon \mapsto \phi(\varepsilon)$ definida em um intervalo aberto contendo $\varepsilon=0$ com $\phi(0)=0$ e $\delta(\phi(\varepsilon), \varepsilon)=0$. Portanto temos o seguinte teorema.

Teorema 2.1.4. Se $\delta_{a}(0,0) \neq 0$, então $\Gamma$ persiste.

Segue de (2.12) que $\sigma(\delta(a, \varepsilon)+a)=\Pi(\sigma(a), \varepsilon)$, assim, derivando com respeito a $a$ e avaliando em $(a, \varepsilon)=(0,0)$, obtemos

$$
\left(\delta_{a}(0,0)+1\right) \dot{\sigma}(0)=\Pi_{a}(\sigma(0), 0) \dot{\sigma}(0) .
$$

Logo, o número $\delta_{a}(0,0)+1$ é uma representação local da derivada da aplicação de Poincaré em $C_{0}$ para $\left\{X_{0}\right\}=\Gamma \cap C_{0}$. Obtemos o seguinte teorema.

Teorema 2.1.5. $\delta_{a}(0,0) \neq 0$ se, e somente se, a derivada da aplicação de Poincaré não é a identidade para $\sigma(0)=X_{0}$.

Uma órbita periódica no plano que satisfaz a propriedade dada no Teorema 2.1.5 é dita hiperbólica. Mais geralmente, uma órbita periódica $\Gamma$ é hiperbólica se a derivada da aplicação de Poincaré para $X_{0}$ não tem autovalores com módulo igual a 1. 
Precisamos de uma fórmula de $\delta_{a}(0,0)$ em termos de $F$. Seja

$$
t \mapsto X(t, Y, \varepsilon)
$$

solução do sistema perturbado (2.7) que satisfaz a condição inicial

$$
X(0, Y, \varepsilon)=Y,
$$

e defina a representação local da função tempo de retorno por

$$
\begin{aligned}
T: \mathbb{R} \times I_{\varepsilon_{0}} & \longrightarrow \mathbb{R} \\
(a, \varepsilon) & \longmapsto T(a, \varepsilon)=\mathcal{T}(\sigma(a), \varepsilon),
\end{aligned}
$$

onde $\mathcal{T}$ e $\sigma$ são dadas em (2.9) e (2.11) respectivamente. Da definição de $\delta$ em (2.12) segue que

$$
\sigma(\delta(a, \varepsilon)+a)=\Pi(\sigma(a), \varepsilon)=X(T(a, \varepsilon), \sigma(a), \varepsilon)
$$

Derivando (2.13) com respeito a $a$ e avaliando em $(a, \varepsilon)=(0,0)$ obtemos

$$
\begin{aligned}
\left(\delta_{a}(0,0)+1\right) \dot{\sigma}(0) & =\dot{X}(T(0,0), \sigma(0), 0) T_{a}(0,0)+D_{2} X(T(0,0), \sigma(0), 0) \dot{\sigma}(0) \\
& =T_{a}(0,0) F\left(X_{0}\right)+D_{2} X\left(2 \pi / \omega, X_{0}, 0\right) \dot{\sigma}(0) .
\end{aligned}
$$

Uma expressão para $\delta_{a}(0,0)$ pode ser obtida da equação (2.14) calculando $D_{2} X\left(2 \pi / \omega, X_{0}, 0\right)$. Segue da dependência diferenciável das soluções com respeito aos parâmetros e condições iniciais que uma solução de (2.7) é diferenciável com respeito a condição inicial $Y$. Tomando a derivada parcial com respeito a $Y$ nas equações

$$
\dot{X}(t, Y, 0)=F(X(t, Y, 0)), \quad X(0, Y, 0)=Y,
$$

a função $t \mapsto D_{2} X(t, Y, 0)=W$ é a matriz de soluções da equação variacional

$$
\dot{W}=D F(X(t, Y, 0)) W
$$

que satisfaz a condição inicial $W(0)=I$ onde $D F$ denota a derivada de $F$. Portanto, $D_{2} X\left(2 \pi / \omega, X_{0}, 0\right)$ é obtido resolvendo o problema de valor inicial variacional para $t=2 \pi / \omega$.

Denotemos por $t \mapsto \Phi(t)$ a matriz fundamental de soluções do sistema (2.15) tal que $\Phi(0)=I$, isto é, $D_{2} X(t, Y, 0)=\Phi(t)$. A matriz $\Phi(t)$ é obtida em seguida.

Temos que $F(Y) \neq(0,0)$ pois $Y \in \Gamma$ e $\Gamma$ é uma órbita periódica, então os vetores $F(X(t, Y, 0))$ e $F^{\perp}(X(t, Y, 0))$ são linearmente independentes para cada $t \in \mathbb{R}$.

Veja que $\Phi(0) F(Y)=F(Y)$ pois $\Phi(0)=I$ e

$$
\frac{\mathrm{d}}{\mathrm{d} t} F(X(t, Y, 0))=D F(X(t, Y, 0)) F(X(t, Y, 0)) .
$$


Logo, $t \mapsto \Phi(t) F(Y)$ e $t \mapsto F(X(t, Y, 0))$ são soluções do mesmo problema de valor inicial. Portanto,

$$
\Phi(t) F(Y)=F(X(t, Y, 0))
$$

Definimos $F^{\perp}=R F$ onde $R$ é a matriz de rotação

$$
R=\left(\begin{array}{rr}
0 & -1 \\
1 & 0
\end{array}\right) .
$$

Como $F$ e $F^{\perp}$ são linearmente independentes em cada ponto do plano onde $F$ não é o vetor nulo, existem duas funções a valores reais $t \mapsto \alpha(t, Y)$ e $t \mapsto \beta(t, Y)$ tal que

$$
\Phi(t) F^{\perp}(Y)=\alpha(t, Y) F(X(t, Y, 0))+\beta(t, Y) F^{\perp}(X(t, Y, 0)) .
$$

Segue das identidades (2.16) e (2.17) que a matriz fundamental $\Phi(t)$ é representada como uma transformação linear de $\mathbb{R}^{2}$, com base $\left\{F(Y), F^{\perp}(Y)\right\}$, para $\mathbb{R}^{2}$, com a base

$$
\left\{F(X(t, Y, 0)), F^{\perp}(X(t, Y, 0))\right\}
$$

pela matriz

$$
\Phi(t)=\left(\begin{array}{cc}
1 & \alpha(t, Y) \\
0 & \beta(t, Y)
\end{array}\right) .
$$

Na equação (2.14), $\dot{\sigma}(0)$ é um vetor tangente a $C_{0}$ em $X_{0}$. Portanto, existem constantes reais $c_{1}$ e $c_{2}$ tal que

$$
\dot{\sigma}(0)=c_{1} F\left(X_{0}\right)+c_{2} F^{\perp}\left(X_{0}\right)
$$

e portanto

$$
\begin{aligned}
& \left(\delta_{a}(0,0)+1\right)\left(c_{1} F\left(X_{0}\right)+c_{2} F^{\perp}\left(X_{0}\right)\right) \\
& =T_{a}(0,0) F\left(X_{0}\right)+\Phi(2 \pi / \omega)\left(c_{1} F\left(X_{0}\right)+c_{2} F^{\perp}\left(X_{0}\right)\right) \\
& =T_{a}(0,0) F\left(X_{0}\right)+c_{1} F\left(X_{0}\right)+c_{2} \alpha\left(2 \pi / \omega, X_{0}\right) F\left(X_{0}\right)+c_{2} \beta\left(2 \pi / \omega, X_{0}\right) F^{\perp}\left(X_{0}\right) .
\end{aligned}
$$

Além disso, como a curva $C_{0}$ é transversal a $\Gamma$, temos que $c_{2} \neq 0$, e como $F$ e $F^{\perp}$ são linearmente independentes, segue que

$$
\begin{aligned}
& \delta_{a}(0,0)=\beta\left(2 \pi / \omega, X_{0}\right)-1, \\
T_{a}(0,0)= & -c_{2} \alpha\left(2 \pi / \omega, X_{0}\right)+c_{1} \delta_{a}(0,0) \\
= & -c_{2} \alpha\left(2 \pi / \omega, X_{0}\right)+c_{1}\left(\beta\left(2 \pi / \omega, X_{0}\right)-1\right) .
\end{aligned}
$$


Observação 2.1.2. As quantidades $\alpha\left(2 \pi / \omega, X_{0}\right)$ e $\beta\left(2 \pi / \omega, X_{0}\right)$ podem ser identificadas geometricamente da seguinte forma: Da equação (2.20), segue que $\beta\left(2 \pi / \omega, X_{0}\right)$ é a representação local da derivada da aplicação de Poincaré do sistema não perturbado (2.8) para $\left\{X_{0}\right\}=\Gamma \cap C_{0}$; Se por exemplo, $t \mapsto \varphi(t)$ é solução da equação $\dot{X}=-F^{\perp}(X)$, com condição inicial $X(0)=X_{0}$, então $\dot{\varphi}(0)=-F^{\perp}\left(X_{0}\right)$. Assim, em (2.19) obtemos $c_{1}=0$ e $c_{2}=-1$. Portanto, na equação (2.21) obtemos que $\alpha\left(2 \pi / \omega, X_{0}\right)$ é a representação local da aplicação tempo de retorno para o sistema não perturbado (2.8) para $X_{0}$ em $C_{0}$.

Expressões para $\alpha(t, Y)$ e $\beta(t, Y)$ em (2.16) foram obtidas primeiro por Stephen P. Diliberto [13. Afim de enunciar o Teorema de Diliberto a seguir, que pode ser encontrado em [11, introduzimos a divergência e o curl para um campo vetorial $F(x, y)=\left(f_{1}(x, y), f_{2}(x, y)\right)$ da seguinte forma:

$$
\begin{aligned}
\operatorname{div} F(x, y) & :=\frac{\partial f_{1}}{\partial x}(x, y)+\frac{\partial f_{2}}{\partial y}(x, y), \\
\operatorname{curl} F(x, y) & :=\frac{\partial f_{2}}{\partial x}(x, y)-\frac{\partial f_{1}}{\partial y}(x, y) .
\end{aligned}
$$

Além disso, dado uma curva $t \mapsto X(t)=(x(t), y(t))$ definimos a função curvatura escalar por

$$
\kappa:=\frac{\dot{x} \ddot{y}-\dot{y} \ddot{x}}{\left(\dot{x}^{2}+\dot{y}^{2}\right)^{3 / 2}} .
$$

Vamos escrever $\kappa(t, Y)$ para denotar a curvatura escalar ao longo da curva $t \mapsto X(t, Y, 0)$ que é uma solução do sistema não perturbado (2.8).

Teorema 2.1.6. (Diliberto) Seja $t \mapsto X(t, Y, 0)$ solução do sistema (2.8) com $F(Y) \neq(0,0)$. Então a matriz fundamental de soluções da equação variacional (2.15) $t \mapsto \Phi(t)$ para $t=0$ é tal que

$$
\begin{aligned}
\Phi(t) F(Y) & =F(X(t, Y, 0)), \\
\Phi(t) F^{\perp}(Y) & =\alpha(t, Y) F(X(t, Y, 0))+\beta(t, Y) F^{\perp}(X(t, Y, 0)),
\end{aligned}
$$

onde

$$
\begin{gathered}
\beta(t, Y)=\frac{\|F(Y)\|^{2}}{\| F^{\perp}\left(X(t, Y, 0) \|^{2}\right.} \cdot \exp \left(\int_{0}^{t} \operatorname{div} F(X(s, Y, 0) \mathrm{d} s),\right. \\
\alpha(t, Y)=\int_{0}^{t}(2 \kappa(s, Y) \| F(X(s, Y, 0) \|-\operatorname{curl} F(X(s, Y, 0))) \beta(s, Y) \mathrm{d} s .
\end{gathered}
$$

Demonstração: As duas primeiras equações foram obtidas logo acima e dadas em (2.16) e (2.17). Por definição

$$
t \mapsto \alpha(t) F(X(t, Y, 0))+\beta(t) F^{\perp}(X(t, Y, 0))
$$

é solução da equação variacional (2.15) com condição inicial $F^{\perp}(Y), \operatorname{logo} \alpha(0)=0$ e $\beta(0)=1$. Derivando esta solução com respeito a $t$ obtemos

$$
\begin{aligned}
& \alpha(t) D F(X(t, Y, 0)) F(X(t, Y, 0))+ \\
& \dot{\alpha}(t) F(X(t, Y, 0))+\beta(t) D F^{\perp}(X(t, Y, 0)) F(X(t, Y, 0))+\dot{\beta}(t) F^{\perp}(X(t, Y, 0)) \\
& =\alpha(t) D F(X(t, Y, 0)) F(X(t, Y, 0))+\beta(t) D F(X(t, Y, 0)) F^{\perp}(X(t, Y, 0)) .
\end{aligned}
$$


Tomando o produto interno com $F^{\perp}(X(t, Y, 0))$ e, para simplificar, omitindo os argumentos das funções obtemos

$$
\dot{\beta}\|F\|^{2}=\beta\left(\left\langle D F \cdot F^{\perp}, F^{\perp}\right\rangle-\left\langle D F^{\perp} \cdot F, F^{\perp}\right\rangle\right) .
$$

Como $F^{\perp}=R F$ onde $R$ é a matriz de rotação

$$
R=\left(\begin{array}{rr}
0 & -1 \\
1 & 0
\end{array}\right)
$$

obtemos

$$
\left\langle D F^{\perp} \cdot F, F^{\perp}\right\rangle=\langle R D F \cdot F, R F\rangle=\langle D F \cdot F, F\rangle
$$

e

$$
\dot{\beta}\|F\|^{2}=\beta\left(\left\langle D F \cdot F^{\perp}, F^{\perp}\right\rangle+\langle D F \cdot F, F\rangle-2\langle D F \cdot F, F\rangle\right) .
$$

Após alguns cálculos, obtemos

$$
\dot{\beta}=\beta \operatorname{div} F-\beta \frac{\mathrm{d}}{\mathrm{d} t} \ln \|F\|^{2} .
$$

A solução desta equação diferencial com condição inicial $\beta(0)=1$ é exatamente a fórmula (2.22).

Na equação (2.24), tomando o produto interno desta vez com $F(X(t, Y, 0))$, obtemos

$$
\begin{aligned}
\dot{\alpha}\|F\|^{2} & =\beta\left(\left\langle D F \cdot F^{\perp}, F\right\rangle-\left\langle D F^{\perp} \cdot F, F\right\rangle\right) \\
& =\beta\left(\left\langle F^{\perp},(D F)^{T} \cdot F\right\rangle-\langle R D F \cdot F, F\rangle\right) \\
& =\beta\left(\left\langle F^{\perp},(D F)^{T} \cdot F\right\rangle+\left\langle F^{\perp}, D F \cdot F\right\rangle\right) \\
& =\beta\left(\left\langle F^{\perp}, 2 D F \cdot F\right\rangle+\left\langle F^{\perp},\left((D F)^{T}-(D F)\right) F\right\rangle\right),
\end{aligned}
$$

onde $T$ significa a transposta. Também, após alguns cálculos, obtemos

$$
\begin{gathered}
\left\langle F^{\perp}, 2 D F \cdot F\right\rangle=2 \kappa\|F\|^{3}, \\
\left\langle F^{\perp}, 2 D F \cdot F\right\rangle+\left\langle F^{\perp},\left((D F)^{T}-(D F)\right) F\right\rangle=-\|F\|^{2} \operatorname{curl} F,
\end{gathered}
$$

onde a curvatura escalar $\kappa$, o curl, e as outras funções são calculadas na curva $t \mapsto X(t, Y, 0)$. Substituindo estas fórmulas em (2.25), e uma integração obtemos (2.23).

Da Observação 2.1 .2 temos que $\beta\left(2 \pi / \omega, X_{0}\right)$ é a representação local da derivada da aplicação de Poincaré. Usando o fato de que $\left\|F\left(X\left(2 \pi / \omega, X_{0}, 0\right)\right)\right\|=\left\|F\left(X_{0}\right)\right\|$ e o Teorema de Diliberto 2.1.6 obtemos

$$
\beta\left(2 \pi / \omega, X_{0}\right)=\exp \left(\int_{0}^{2 \pi / \omega} \operatorname{div} F\left(X\left(t, X_{0}, 0\right) \mathrm{d} t\right) .\right.
$$

Lembrando que uma órbita periódica $\Gamma$ é hiperbólica se a derivada da aplicação de Poincaré calculada em $C_{0}$ para $\left\{X_{0}\right\}=\Gamma \cap C_{0}$ não tem autovalores com módulo igual a 1 , e como a 
expressão de $\beta\left(2 \pi / \omega, X_{0}\right)$ não depende de $C_{0}$, temos que a derivada da aplicação de Poincaré não depende da escolha da seção de Poincaré $C_{0}$. Além disso, por uma mudança de variáveis, a derivada da aplicação de Poincaré não depende também do ponto $X_{0} \in \Gamma$.

Proposição 2.1.1. Uma solução periódica $t \mapsto X(t, Y, 0)$ do sistema não perturbado (2.8) com período $2 \pi / \omega$ é hiperbólica se, e somente se,

$$
\int_{0}^{2 \pi / \omega} \operatorname{div} F(X(t, Y, 0)) \mathrm{d} t \neq 0
$$

Observação 2.1.3. Para determinar a persistência de órbitas periódicas do sistema não perturbado (2.8), a nossa principal hipótese, $\delta_{a}(0,0) \neq 0$, é equivalente a exigir que a solução periódica deste sistema é hiperbólica. Agora, se uma órbita periódica não é hiperbólica, então não podemos determinar uma solução implícita da equação $\delta(a, \varepsilon)=0$ por uma aplicação direta do Teorema da Função Implícita. Em vez disso, a principal ferramenta para essa nova análise é o Teorema de Preparação (Weierstrass) ver [1].

Suponhamos agora, que $\Gamma$ está contida num região anular $\mathcal{A}$ de órbitas periódicas, isto é, uma região anular do plano que consiste inteiramente de órbitas periódicas do sistema não perturbado (2.8). Assim, temos que $\delta(a, 0) \equiv 0$. Vamos considerar todas as órbitas periódicas na região anular $\mathcal{A}$ e determinar se algumas destas órbitas persistem. Os passos para isso são os mesmos dados na continuação das órbitas periódicas do centro linear perturbado (2.1). Para este problema, obtemos o mesmo Teorema 2.1.1, que, no presente contexto é o seguinte:

Teorema 2.1.7. Se $a_{0}$ é um zero simples da função $a \mapsto \delta_{\varepsilon}(a, 0)$, isto é, $\delta_{\varepsilon}\left(a_{0}, 0\right)=0$ e $\delta_{a \varepsilon}\left(a_{0}, 0\right) \neq 0$, então a órbita periódica $\Gamma_{a_{0}}$ do sistema não perturbado (2.8) com valor inicial $\sigma\left(a_{0}\right) \in C_{0}$ persiste.

Além disso, o Teorema 2.1.2 repete-se neste caso mais geral da mesma forma.

Observação 2.1.4. O problema de persistência e estabilidade para $\varepsilon \geq 0$ é equivalente aqui a dizer que, se $a_{0}$ é um zero simples da função $\delta_{\varepsilon}(a, 0)$, então para $\varepsilon>0$ suficientemente pequeno existe um ciclo limite $X^{\varepsilon}$ de (2.7) tal que $X^{\varepsilon}$ tende à órbita periódica $\Gamma_{a_{0}}$ quando $\varepsilon$ tende a 0 . $O$ ciclo limite é estável se $\delta_{a \varepsilon}\left(a_{0}, 0\right)<0$ e instável se $\delta_{a \varepsilon}\left(a_{0}, 0\right)>0$.

Suponhamos daqui por diante que $\varepsilon \geq 0$ no sistema (2.7). Precisamos encontrar uma fórmula para $\delta_{\varepsilon}(a, 0)$, para isso, inicialmente, vamos derivar a equação (2.13) com respeito a $\varepsilon$ e avaliar em $(a, 0)$, obtendo

$$
\delta_{\varepsilon}(a, 0) \dot{\sigma}(a)=T_{\varepsilon}(a, 0) F(\sigma(a))+X_{\varepsilon}(T(a, 0), \sigma(a), 0) .
$$


Teorema 2.1.8. (Lema Variacional)

$$
\begin{aligned}
X_{\varepsilon}(T(a, 0), \sigma(a), 0) & =(\mathcal{N}(a)+\alpha(T(a, 0), \sigma(a)) \mathcal{M}(a)) F(\sigma(a)) \\
& +\beta(T(a, 0), \sigma(a)) \mathcal{M}(a) F^{\perp}(\sigma(a)),
\end{aligned}
$$

onde

$$
\begin{aligned}
\mathcal{M}(a)= & \int_{0}^{T(a, 0)} \frac{F(X(t, \sigma(a), 0)) \wedge G(X(t, \sigma(a), 0))}{\beta(t, \sigma(a))\|F(X(t, \sigma(a), 0))\|^{2}} \mathrm{~d} t \\
= & \frac{1}{\|F(\sigma(a))\|^{2}} \int_{0}^{T(a, 0)} e^{-\int_{0}^{t} \operatorname{div} F(X(s, \sigma(a), 0)) \mathrm{d} s} F(X(t, \sigma(a), 0)) \wedge G(X(t, \sigma(a), 0)) \mathrm{d} t \\
& \mathcal{N}(a)=\int_{0}^{T(a, 0)} \frac{\langle G(X(t, \sigma(a), 0)), F(X(t, \sigma(a), 0))\rangle}{\|F(X(t, \sigma(a), 0))\|^{2}} \mathrm{~d} t \\
& -\int_{0}^{T(a, 0)} \frac{\alpha(t, \sigma(a)) \cdot(F(X(t, \sigma(a), 0)) \wedge G(X(t, \sigma(a), 0)))}{\beta(t, \sigma(a))\|F(X(t, \sigma(a), 0))\|^{2}} \mathrm{~d} t
\end{aligned}
$$

com $\alpha$ e $\beta$ dadas em (2.23) e (2.22) respectivamente.

Demonstração: Da dependência diferenciável das soluções com respeito aos parâmetros e condições iniciais uma solução de (2.7) é diferenciável com respeito ao parâmetro $\varepsilon$. Tomando a derivada parcial com respeito a $\varepsilon$ nas equações

$$
\dot{X}(t, Y, \varepsilon)=F(X(t, Y, \varepsilon))+\varepsilon G(X(t, Y, \varepsilon)), \quad X(0, Y, \varepsilon)=Y
$$

obtemos que a função $t \mapsto X_{\varepsilon}(t, \sigma(a), 0)$ é contínua e solução do problema de valor inicial variacional não homogêneo

$$
\left\{\begin{array}{l}
\dot{W}=D F(X(t, \sigma(a), 0)) W+G(X(t, \sigma(a), 0)), \\
W(0)=0 .
\end{array}\right.
$$

Pelo Método da Variação de Parâmetros, obtemos

$$
X_{\varepsilon}(T(a, 0), \sigma(a), 0)=\Phi(T(a, 0)) \int_{0}^{T(a, 0)} \Phi^{-1}(s) G(X(s, \sigma(a), 0) \mathrm{d} s,
$$

onde $\Phi(t)$ denota a matriz fundamental de soluções do sistema (2.15) para $t=0$.

Agora, a função $t \mapsto X(t, \sigma(a), 0)$ é solução de

$$
\dot{X}(t, Y, 0)=F(X(t, Y, 0))
$$

assim o campo $G\left(X(t, \sigma(a), 0)\right.$ pode ser escrito na base $\left\{F, F^{\perp}\right\}$ para $X(t, \sigma(a), 0)$ na forma

$$
G\left(X(t, \sigma(a), 0)=c_{1}(t, \sigma(a)) F\left(X(t, \sigma(a), 0)+c_{2}(t, \sigma(a)) F^{\perp}(X(t, \sigma(a), 0) .\right.\right.
$$


Tomando o produto interno de $F$ e $F^{\perp}$ em ambos os membros de (2.31) obtemos respectivamente $c_{1}$ e $c_{2}$ dados por

$$
\begin{array}{r}
c_{1}(t, \sigma(a))=\frac{1}{\|F(X(t, \sigma(a), 0))\|^{2}}\langle G(X(t, \sigma(a), 0)), F(X(t, \sigma(a), 0))\rangle, \\
c_{2}(t, \sigma(a))=\frac{1}{\left\|F^{\perp}(X(t, \sigma(a), 0))\right\|^{2}}\left\langle G(X(t, \sigma(a), 0)), F^{\perp}(X(t, \sigma(a), 0))\right\rangle \\
=\frac{1}{\|F(X(t, \sigma(a), 0))\|^{2}} F(X(t, \sigma(a), 0)) \wedge G(X(t, \sigma(a), 0)),
\end{array}
$$

onde $F \wedge G=f_{1} g_{2}-f_{2} g_{1}$. A última igualdade em (2.33) decorre do fato de que $F^{\perp}=$ $\left(-f_{2}, f_{1}\right)$ e $\left\langle G, F^{\perp}\right\rangle=f_{1} g_{2}-f_{2} g_{1}$. Além disso, note que a inversa da matriz (2.18) representa a ação da inversa da matriz fundamental para $t=0$ a partir do conjunto gerado por $\left\{F(X(t, \sigma(a), 0)), F^{\perp}(X(t, \sigma(a), 0))\right\}$ para o conjunto gerado por $\left\{F(X(\sigma(a))), F^{\perp}(X(\sigma(a)))\right\}$. Do mesmo modo, a matriz (2.18) avaliada em $T(a, 0)$ é a representação matricial da matriz fundamental com respeito à base $\left\{F(X(\sigma(a))), F^{\perp}(X(\sigma(a)))\right\}$. Segue destas observações que

$$
\begin{gathered}
\Phi(T(a, 0))=\left(\begin{array}{cc}
1 & \alpha(T(a, 0), \sigma(a)) \\
0 & \beta(T(a, 0), \sigma(a))
\end{array}\right), \\
\Phi^{-1}(s) G(X(s, \sigma(a), 0))=\frac{1}{\beta(s, \sigma(a))}\left(\begin{array}{cc}
\beta(s, \sigma(a)) & -\alpha(s, \sigma(a)) \\
0 & 1
\end{array}\right)\left(\begin{array}{l}
c_{1}(s, \sigma(a)) \\
c_{2}(s, \sigma(a))
\end{array}\right) .
\end{gathered}
$$

Substituindo (2.34) e (2.35) em (2.30) obtemos

$$
\begin{aligned}
X_{\varepsilon}(T(a, 0), \sigma(a), 0) & =(\mathcal{N}(a)+\alpha(T(a, 0), \sigma(a)) \mathcal{M}(a)) F(\sigma(a)) \\
& +\beta(T(a, 0), \sigma(a)) \mathcal{M}(a) F^{\perp}(\sigma(a)),
\end{aligned}
$$

onde $\mathcal{M}$ e $\mathcal{N}$ são as funções dadas em (2.28) (2.29).

Substituindo (2.27) em (2.26) e depois disso tomando o produto interno de ambos os membros da equação (2.26) com o vetor $F^{\perp}(\sigma(a))$ obtemos

$$
\delta_{\varepsilon}(a, 0)=\frac{\beta(T(a, 0), \sigma(a))\|F(\sigma(a))\|^{2}}{\left\langle\dot{\sigma}(a), F^{\perp}(\sigma(a))\right\rangle} \mathcal{M}(a) .
$$

Nesta fórmula, $\left\langle\dot{\sigma}, F^{\perp}\right\rangle \neq 0$ porque $C_{0}$ é transversal às soluções periódicas do sistema perturbado (2.7), e $\beta(t, Y) \neq 0$ porque $\|F\|$ não se anula ao longo de uma solução periódica do sistema perturbado (2.7).

Definição 2.1.2. Na equação (2.36) a função definida por

$$
\mathcal{M}(a)=\int_{0}^{T(a, 0)} e^{-\int_{0}^{t} \operatorname{div} F(X(s, \sigma(a), 0)) \mathrm{d} s} F(X(t, \sigma(a), 0)) \wedge G(X(t, \sigma(a), 0)) \mathrm{d} t
$$

é chamada de Função de Poincaré-Andronov-Melnikov autônoma ou simplesmente de Função de Melnikov de primeira ordem. 
Teorema 2.1.9. Suponha que o sistema não perturbado (2.8) tem uma região anular $\mathcal{A}$ de órbitas periódicas $\Gamma_{a}$ com orientação horária e parametrizadas por $t \mapsto X(t, \sigma(a), 0)$, onde $\sigma$ é função suave dada em (2.11). Se $a_{0}$ é um zero simples da função $a \mapsto \mathcal{M}(a)$ dada pela fórmula (2.37) para o sistema perturbado (2.7), então para $\varepsilon>0$ suficientemente pequeno existe um ciclo limite $X^{\varepsilon}$ de (2.7) tal que $X^{\varepsilon}$ tende à órbita periódica $\Gamma_{a_{0}}$ do sistema (2.8) quando $\varepsilon$ tende a 0 . O ciclo limite é estável se $\mathcal{M}^{\prime}\left(a_{0}\right)<0$ e instável se $\mathcal{M}^{\prime}\left(a_{0}\right)>0$.

Demonstração: Este resultado segue imediatamente a partir da fórmula 2.36 e do fato de que zeros simples de $a \mapsto \delta_{\varepsilon}(a, 0)$ persistem. Veja Observação 2.1.4. Além disso, observe que, em geral, dadas funções reais $f, g$ e $h$ tais que $f(a)=g(a) h(a)$ com $g(a)$ não nulo, então os zeros simples de $f$ e $h$ coincidem.

Observação 2.1.5. Se no Teorema 2.1.9 tivermos $\Gamma_{a}$ com orientação anti-horária, na Definição 2.1.2 tome

$$
\mathcal{M}(a)=-\int_{0}^{T(a, 0)} e^{-\int_{0}^{t} \operatorname{div} F(X(s, \sigma(a), 0)) \mathrm{d} s} F(X(t, \sigma(a), 0)) \wedge G(X(t, \sigma(a), 0)) \mathrm{d} t .
$$

Considere o sistema Hamiltoniano perturbado

$$
\left\{\begin{array}{l}
\dot{x}=-H_{y}(x, y)+\varepsilon g_{1}(x, y), \\
\dot{y}=H_{x}(x, y)+\varepsilon g_{2}(x, y),
\end{array}\right.
$$

onde $H, g_{1}, g_{2} \in C^{k}, k \geq 1$ e $\varepsilon \geq 0$ parâmetro real. Para $\varepsilon=0$, suponha que existe uma família de órbitas periódicas $\Gamma_{a}$ com orientação anti-horária e definida por

$$
\Gamma_{a}: H(x, y)=a, a>0
$$

parametrizadas por $\gamma_{a}(t)$ de período $T(a, 0)>0$ com $t \in[0, T(a, 0)]$.

Teorema 2.1.10. Se a $>0$ é um zero simples da função integral $\mathcal{M}:(0, \infty) \rightarrow \mathbb{R}$ dada por

$$
a \mapsto \mathcal{M}(a)=\oint_{\gamma_{a}} g_{1} \mathrm{~d} y-g_{2} \mathrm{~d} x
$$

então para $\varepsilon>0$ suficientemente pequeno existe um ciclo limite $X^{\varepsilon}$ de (2.39) tal que $X^{\varepsilon}$ tende a $\gamma_{a}$ quando $\varepsilon$ tende a 0 . O ciclo limite é estável se $\mathcal{M}^{\prime}(a)<0$ e instável se $\mathcal{M}^{\prime}(a)>0$.

Demonstração: Como o sistema (2.39) para $\varepsilon=0$ é Hamiltoniano, temos que $\operatorname{div} F=0$ onde $F=\left(-H_{y}, H_{x}\right)$. Pela Observação 2.1.5 a função de Melnikov de primeira ordem $\mathcal{M}(a)$ é dada por

$$
\mathcal{M}(a)=-\int_{0}^{T(a, 0)} F\left(\gamma_{a}(t)\right) \wedge G\left(\gamma_{a}(t)\right) \mathrm{d} t
$$


Temos portanto que

$$
\mathcal{M}(a)=\int_{0}^{T(a, 0)}\left(H_{y} g_{2}+H_{x} g_{1}\right)\left(\gamma_{a}(t)\right) \mathrm{d} t
$$

Agora, veja que

$$
\begin{aligned}
\int_{\gamma_{a}} g_{1} \mathrm{~d} y-g_{2} \mathrm{~d} x & =\int_{0}^{T(a, 0)}\left(-g_{2}, g_{1}\right)\left(\gamma_{a}(t)\right) \cdot \gamma_{a}^{\prime}(t) \mathrm{d} t \\
& =\int_{0}^{T(a, 0)}\left(\left(-g_{2}, g_{1}\right) \cdot\left(-H_{y}, H_{x}\right)\right)\left(\gamma_{a}(t)\right) \cdot \gamma_{a}^{\prime}(t) \mathrm{d} t \\
& =\int_{0}^{T(a, 0)}\left(H_{y} g_{2}+H_{x} g_{1}\right)\left(\gamma_{a}(t)\right) \mathrm{d} t
\end{aligned}
$$

Portanto,

$$
\mathcal{M}(a)=\oint_{\gamma_{a}} g_{1} \mathrm{~d} y-g_{2} \mathrm{~d} x
$$

A segunda parte segue do Teorema 2.1.9.

Observação 2.1.6. Se o sistema (2.39) é tal que $H_{y}=1$ e $H_{x}=1$, temos um centro linear perturbado. Neste caso a família de órbitas periódicas pode ser parametrizada por $\gamma_{a}(t)=$ $(a \cos (t), a \operatorname{sen}(t))$, com $t \in[0,2 \pi]$ com período $T(a, 0)=2 \pi$ e orientação anti-horária. Assim da equação 2.40, obtemos

$$
\mathcal{M}(a)=\oint_{\gamma_{a}} g_{1} \mathrm{~d} y-g_{2} \mathrm{~d} x=a \int_{0}^{2 \pi} g_{1}(a \cos (t), a \operatorname{sen}(t)) \cos (t)+g_{2}(a \cos (t), a \operatorname{sen}(t)) \operatorname{sen}(t) \mathrm{d} t .
$$

Observe que a expressão integral acima é a mesma dada no Teorema 2.1.3.

\subsection{Regularização de Campos Descontínuos}

Nesta seção vamos apresentar o método de regularização de campos descontínuos e as convenções de Filippov.

\subsubsection{Método de Regularização}

Sejam $X^{+}$e $X^{-}$campos de classe $C^{k}, k \geq 1$ em $K \subset \mathbb{R}^{2}$ compacto e conexo e $\mathcal{H}: K \rightarrow \mathbb{R}$ aplicação real suave definida em $K$ tal que 0 é valor regular. Suponhamos que $\Sigma=\mathcal{H}^{-1}(0)$ é compacto e conexo tal que $K-\Sigma$ tem exatamente duas componentes conexas que são

$$
\Sigma^{-}=\mathcal{H}^{-1}(-\infty, 0) \quad \text { e } \quad \Sigma^{+}=\mathcal{H}^{-1}(0,+\infty)
$$

O conjunto $\Sigma$ é chamado de curva de separação ou curva de descontinuidade. 


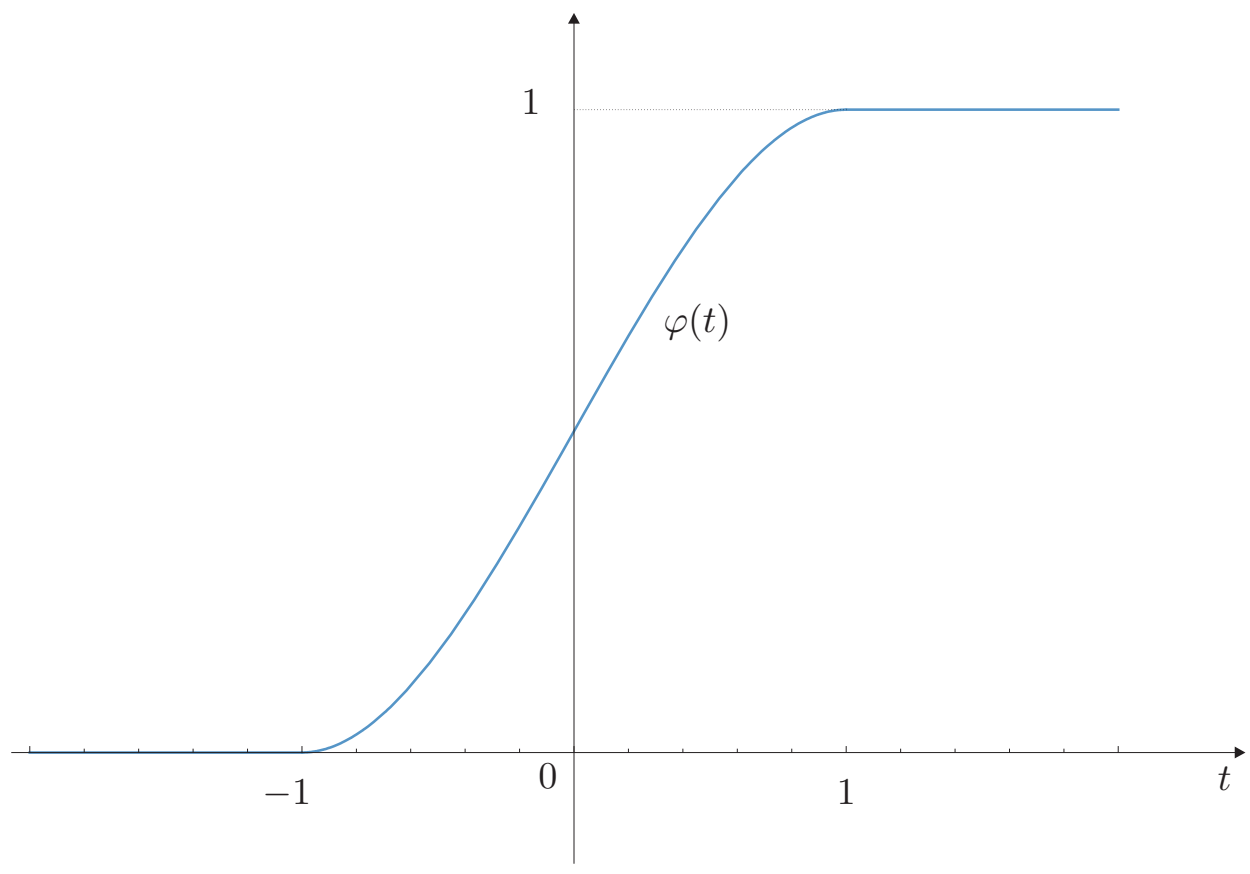

Figura 2.1: Gráfico da Função de Transição.

Definição 2.2.1. Definimos o campo vetorial descontínuo $Z=\left(X^{-}, X^{+}\right)$em $K$ por

$$
Z(x, y)= \begin{cases}X^{-}(x, y), & \mathcal{H}(x, y) \leq 0 \\ X^{+}(x, y), & \mathcal{H}(x, y) \geq 0\end{cases}
$$

Seja $\varphi: \mathbb{R} \rightarrow \mathbb{R}$ uma função (de transição) de classe $C^{k}, k \geq 1$ (veja Figura 2.1) definida da seguinte forma

$$
\varphi(t)= \begin{cases}0, & t \leq-1 \\ 1, & t \geq 1\end{cases}
$$

$\varphi^{\prime}(t)>0$ em $(-1,1)$. Além disso, definimos $\varphi^{\mu}(t)=\varphi(t / \mu)$ para todo $t \in \mathbb{R}$ e um número real $\mu>0$, chamado de parâmetro de regularização. Chamamos $\varphi^{\mu}$ de função de regularização.

Conforme Sotomayor e Teixeira [38], a regularização do campo $Z$ é dada pela família a um parâmetro de campos vetoriais suaves

$$
Z^{\mu}(x, y)=\left(1-\varphi^{\mu}(\mathcal{H}(x, y))\right) X^{-}(x, y)+\varphi^{\mu}(\mathcal{H}(x, y)) X^{+}(x, y)
$$

Obtemos uma faixa em torno da curva $\Sigma$ chamada de faixa de regularização dada por $\left(\varphi^{\mu} \circ\right.$ $\mathcal{H})^{-1}(0,1)$, ou seja, o conjunto dos pontos $(x, y) \in \mathbb{R}^{2}$ tal que $\varphi^{\mu}(\mathcal{H}(x, y)) \in(0,1)$. A faixa de regularização é a faixa onde o campo $Z^{\mu}$ em (2.42), para cada $\mu>0$ fixado é definido por uma "média" entre os campos $X^{+}$e $X^{-}$e faz com que o campo $Z^{\mu}$ seja suave, ou seja, a regularização ocorre nesta faixa. Agora, fora desta faixa, se $\mathcal{H}(x, y) \geq \mu$, então, $\varphi^{\mu}(\mathcal{H}(x, y))=1$, $\operatorname{logo} Z^{\mu}(x, y)=X^{+}(x, y)$ e se $\mathcal{H}(x, y) \leq-\mu$, então, $\varphi^{\mu}(\mathcal{H}(x, y))=0, \operatorname{logo} Z^{\mu}(x, y)=X^{-}(x, y)$. 
Seja $\Sigma^{j} \subseteq \Sigma$, onde $\Sigma^{j}$ é um conjunto aberto tal que, para todo $X \in \Sigma^{j}$ temos

$$
X^{-}(X) \neq X^{+}(X)
$$

chamamos o sistema associado ao campo descontínuo (2.41) de sistema suave por partes com duas zonas no plano descontínuo. Agora, se para todo $X \in \Sigma$ a igualdade ocorre em (2.43), chamamos o sistema associado ao campo (2.41) de sistema suave por partes com duas zonas no plano contínuo. Em todo caso o método de regularização pode ser aplicado. Se vale (2.43) estamos dizendo que o campo $Z$ em (2.41) é bi-valuado em $\Sigma^{j}$. Se um ponto $X_{0}$ está em $\Sigma^{+}$ou $\Sigma^{-}$a órbita é a usual dos campos vetoriais suaves $X^{+}$ou $X^{-}$. Em seu livro clássico Filippov [14] introduziu como pode ocorrer a transição das órbitas entre as regiões $\Sigma^{+}$e $\Sigma^{-}$, ou, se for o caso, de como as órbitas podem permanecer sobre $\Sigma$, chamadas de Convenções de Filippov. Filippov introduziu o que vem a ser em $\Sigma$ os pontos de costura, escape, deslize ou pontos de tangência.

Definição 2.2.2. (Convenções de Filippov) Considere o campo (2.41) e um ponto $X_{0} \in \Sigma$ tal que $X^{-}\left(X_{0}\right) \neq 0$ e $X^{+}\left(X_{0}\right) \neq 0$.

(a) Um conjunto $\Sigma^{C} \subseteq \Sigma$ é chamado de conjunto ou região de costura, se para todo $X_{0} \in \Sigma^{C}$ tivermos

$$
\left(X^{-}\left(X_{0}\right) \cdot \nabla \mathcal{H}\left(X_{0}\right)\right)\left(X^{+}\left(X_{0}\right) \cdot \nabla \mathcal{H}\left(X_{0}\right)\right)>0
$$

Chamamos $X_{0} \in \Sigma^{C}$ de ponto de costura. Veja Figura (2.2).

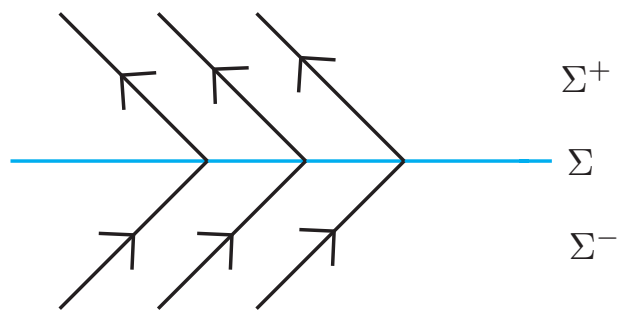

Figura 2.2: Arco de costura.

(b) Um conjunto $\Sigma^{E} \subseteq \Sigma$ é chamado de conjunto ou região de escape, se para todo $X_{0} \in \Sigma^{C}$ tivermos

$$
\left(X^{-}\left(X_{0}\right) \cdot \nabla \mathcal{H}\left(X_{0}\right)\right)<0 \quad \text { e } \quad\left(X^{+}\left(X_{0}\right) \cdot \nabla \mathcal{H}\left(X_{0}\right)\right)>0 .
$$

Chamamos $X_{0} \in \Sigma^{E}$ de ponto de escape. Veja Figura (2.3).

(c) Um conjunto $\Sigma^{D} \subseteq \Sigma$ é chamado de conjunto ou região de deslize, se para todo $X_{0} \in \Sigma^{D}$ tivermos

$$
\left(X^{-}\left(X_{0}\right) \cdot \nabla \mathcal{H}\left(X_{0}\right)\right)>0 \quad e \quad\left(X^{+}\left(X_{0}\right) \cdot \nabla \mathcal{H}\left(X_{0}\right)\right)<0 .
$$

Chamamos $X_{0} \in \Sigma^{D}$ de ponto de deslize. Veja Figura (2.4). 


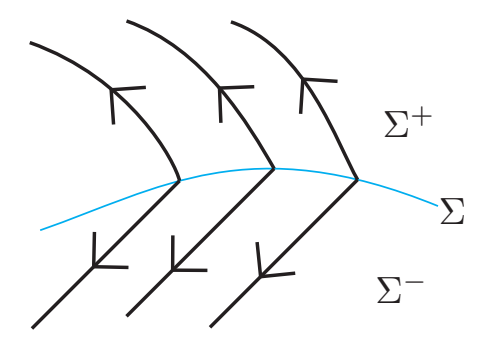

Figura 2.3: Arco de escape.

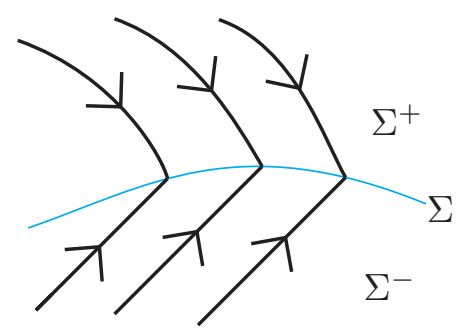

Figura 2.4: Arco de deslize.

Definição 2.2.3. $X_{0} \in \Sigma$ é ponto de tangência (na fronteira), se $\|\left(X^{-}\left(X_{0}\right)\|+\| X^{+}\left(X_{0}\right) \| \neq 0\right.$ $e$

$$
\left(X^{-}\left(X_{0}\right) \cdot \nabla \mathcal{H}\left(X_{0}\right)\right)\left(X^{+}\left(X_{0}\right) \cdot \nabla \mathcal{H}\left(X_{0}\right)\right)=0
$$

Em particular, para a região de costura, toda órbita atravessa $\Sigma^{C}$ transversalmente. Nas regiões de escape ou deslize define-se um campo auxiliar chamado de Campo de Filippov. Para mais detalhes ver [30] e 31].

\subsubsection{Aplicação do Método de Regularização}

Considere o campo Z em $\mathbb{R}^{2}$ dado por

$$
Z(X, \varepsilon)= \begin{cases}X^{-}(X, \varepsilon), & \mathcal{H}(X) \leq 0 \\ X^{+}(X, \varepsilon), & \mathcal{H}(X) \geq 0\end{cases}
$$

e definido pelo sistema

$$
\dot{X}= \begin{cases}F(X)+\varepsilon G^{-}(X), & \mathcal{H}(X) \leq 0 \\ F(X)+\varepsilon G^{+}(X), & \mathcal{H}(X) \geq 0\end{cases}
$$

onde $X=(x, y) \in \mathbb{R}^{2}, \varepsilon \geq 0$ é um parâmetro real, $F(x, y)=\left(f_{1}(x, y), f_{2}(x, y)\right)$ e

$$
\begin{aligned}
G: & \mathbb{R}^{2} \longrightarrow \mathbb{R}^{2} \\
X & \longmapsto G(X)= \begin{cases}G^{-}(X), & \mathcal{H}(X) \leq 0 \\
G^{+}(X), & \mathcal{H}(X) \geq 0,\end{cases}
\end{aligned}
$$


sendo $F, G^{-}(x, y)=\left(g_{1}^{-}(x, y), g_{2}^{-}(x, y)\right)$ e $G^{+}(x, y)=\left(g_{1}^{+}(x, y), g_{2}^{+}(x, y)\right)$ campos vetoriais de classe $C^{k}, k \geq 1$.

Regularizando (2.44) obtemos o campo

$$
Z^{\mu}(x, y, \varepsilon)=\left(1-\varphi^{\mu}(\mathcal{H}(x, y))\right) X^{-}(x, y)+\varphi^{\mu}(\mathcal{H}(x, y)) X^{+}(x, y)
$$

então,

$$
Z^{\mu}(X, \varepsilon)=F(X)+\varepsilon R(X, \mu)
$$

onde

$$
R(X, \mu)=R(x, y, \mu)=\left(r_{1}(x, y, \mu), r_{2}(x, y, \mu)\right)
$$

com

$$
r_{1}(x, y, \mu)=g_{1}^{-}(x, y)+\varphi^{\mu}(\mathcal{H}(x, y))\left(g_{1}^{+}(x, y)-g_{1}^{-}(x, y)\right)
$$

e

$$
r_{2}(x, y, \mu)=g_{2}^{-}(x, y)+\varphi^{\mu}(\mathcal{H}(x, y))\left(g_{2}^{+}(x, y)-g_{2}^{-}(x, y)\right) .
$$

Teorema 2.2.1. Suponha que o sistema (2.45) tem uma região anular $\mathcal{A}$ de órbitas periódicas $\Gamma_{a}^{\mu}$ para $\varepsilon=0$ com orientação horária, período $T(a, 0)>0$ e parametrizadas por $t \mapsto X(t, \sigma(a), 0)$, onde $\sigma$ é função suave dada em (2.11). Para cada $\mu>0$, se $a_{0}$ é um zero simples da função

$$
\mathcal{R}(a, \mu)=\int_{0}^{T(a, 0)} e^{-\int_{0}^{t} \operatorname{div} F(X(s, \sigma(a), 0)) \mathrm{d} s} F(X(t, \sigma(a), 0)) \wedge R(X(t, \sigma(a), 0), \mu) \mathrm{d} t
$$

chamada de Função de Melnikov de primeira ordem do sistema regularizado, então para $\varepsilon>0$ suficientemente pequeno existe um ciclo limite $X^{\varepsilon, \mu}$ de (2.46) tal que $X^{\varepsilon, \mu}$ tende a $\Gamma_{a_{0}}^{\mu}$ quando $\varepsilon$ tende a 0 . O ciclo limite é estável se $\mathcal{R}_{a}\left(a_{0}, \mu\right)<0$ e instável se $\mathcal{R}_{a}\left(a_{0}, \mu\right)>0$.

Demonstração: Para cada $\mu>0$ o campo regularizado $Z^{\mu}$ em (2.46) é suave, portanto podemos aplicar a teoria suave. Além disso, $Z^{\mu}$ é uma perturbação do campo definido pelo sistema $\dot{X}=F(X)$. Então para cada $\mu>0$ a função de Melnikov é dada na Definição 2.37, isto é, a função de Melnikov de primeira ordem para um elemento da família $Z^{\mu}$ é dada por (2.47). Segue do Teorema 2.1.9 que para cada $\mu>0$, podemos obter os ciclos limites (e sua estabilidade) que bifurcam do anel $\mathcal{A}$ de órbitas periódicas do sistema regularizado dado pelo campo (2.46).

Observação 2.2.1. Se no Teorema 2.2.1 tivermos $\Gamma_{a}^{\mu}$ com orientação anti-horária, tome

$$
\mathcal{R}(a, \mu)=-\int_{0}^{T(a, 0)} e^{-\int_{0}^{t} \operatorname{div} F(X(s, \sigma(a), 0)) \mathrm{d} s} F(X(t, \sigma(a), 0)) \wedge R(X(t, \sigma(a), 0), \mu) \mathrm{d} t .
$$




\section{Capítulo 3}

\section{Sistemas Lineares Hurwitzianos por}

\section{Partes}

Neste capítulo vamos estudar a estabilidade assintótica global de sistemas lineares por partes Hurwitzianos com duas zonas no plano tendo pontos na reta de separação das duas zonas do tipo costura, exceto a origem, que é o único ponto de equilíbrio dos sistema. Além disso, mudando a reta de separação por uma curva poligonal, mostramos que podem existir matrizes Hurwitz tais que origem seja instável. As principais referências deste capítulo são [4] e [15].

\subsection{Estabilidade de Sistemas Lineares Hurwitzianos com Duas Zonas no Plano}

Sistemas de equações diferenciais ordinárias lineares por partes com duas zonas no plano são geralmente definidos por

$$
\dot{X}= \begin{cases}A^{-} X+B^{-}, & \mathcal{H}(X) \leq 0, \\ A^{+} X+B^{+}, & \mathcal{H}(X) \geq 0,\end{cases}
$$

onde o ponto denota a derivada com respeito a variável independente $t$, chamada aqui de tempo, $X=(x, y) \in \mathbb{R}^{2}, A^{ \pm}$são matrizes reais $2 \times 2, B^{ \pm}$são matrizes reais $2 \times 1$, a função $\mathcal{H}: \mathbb{R}^{2} \rightarrow \mathbb{R}$ é pelo menos contínua e o conjunto $\Sigma=\mathcal{H}^{-1}(0)$ divide o plano em duas componentes (zonas) ilimitadas $\Sigma^{+}$e $\Sigma^{-}$onde $\mathcal{H}$ é positiva e negativa respectivamente. Assim $\mathbb{R}^{2}=\Sigma^{+} \cup \Sigma \cup \Sigma^{-}$.

Em [15] os autores analisaram o caso em que:

H1. $A^{+}$e $A^{-}$são matrizes Hurwitzianas (a parte real de todos os autovalores é negativa);

H2. $B^{+}=B^{-}=0$; 
H3. o conjunto de separação $\Sigma$ é uma reta que contém um único ponto de equilíbrio na origem do sistema;

H4. os campos de vetores $A^{+} X$ e $A^{-} X$ são contínuos em $\Sigma-\{(0,0)\}$.

Com as hipóteses acima, em [15] provou-se que o único ponto de equilíbrio é globalmente assintoticamente estável.

Nesta tese, vamos estender o resultado sobre a estabilidade assintótica global mudando a hipótese $\mathbf{H} 4$ à seguinte:

H4'. Os pontos em $\Sigma-\{(0,0)\}$ são do tipo costura.

Considere o seguinte teorema.

Teorema 3.1.1. Considere o sistema (3.1) com as hipóteses H1, H2, H3 e H4'. Então o único ponto de equilíbrio que é a origem é globalmente assintoticamente estável.

Além disso, o conjunto de separação $\Sigma$ entre as duas zonas desempenha um papel importante nos sistemas diferenciais lineares planares seccionalmente descontínuos.

Considere a hipótese:

H3'. o conjunto de separação $\Sigma$ é uma curva poligonal dada pela união de duas semi-retas que contêm um único ponto de equilíbrio na origem do sistema.

Provamos também, o seguinte teorema.

Teorema 3.1.2. Considere o sistema (3.1) com as hipóteses H1, H2, H3' and H4'. Existem matrizes Hurwitzianas $A^{+}$e $A^{-}$tal que o único ponto de equilíbrio na origem é ou um foco estável, ou um centro, ou um foco instável.

\subsection{Conjunto de Separação é uma Reta}

\section{Prova do Teorema 3.1.1}

Sem perda de generalidade, podemos assumir que $\mathcal{H}(x, y)=y$, assim o conjunto de separação é dado por

$$
\Sigma=\left\{(x, y) \in \mathbb{R}^{2}: y=0\right\}
$$

e

$$
\Sigma^{+}=\left\{(x, y) \in \mathbb{R}^{2}: y>0\right\}, \quad \Sigma^{-}=\left\{(x, y) \in \mathbb{R}^{2}: y<0\right\} .
$$


Lema 3.2.1. Considere os sistemas

$$
\dot{X}=\left\{\begin{array}{c}
\widetilde{A}^{-} X, \quad y \leq 0 \\
\widetilde{A}^{+} X, \quad y \geq 0
\end{array}\right.
$$

onde $\widetilde{A}^{ \pm}=\left(\widetilde{a}_{i j}^{ \pm}\right)$são matrizes Hurwitzianas e os pontos em $\Sigma-\{(0,0)\}$ são do tipo costura. Então existe uma mudança de coordenadas linear preservando os pontos de costura em $\Sigma-\{(0,0)\}$ tal que os sistemas têm a forma

$$
\dot{X}=\left\{\begin{array}{l}
A^{-} X, \quad y \leq 0 \\
A^{+} X, \quad y \geq 0
\end{array}\right.
$$

onde

$$
A^{ \pm}=\left(\begin{array}{cc}
\sigma^{ \pm} & \delta^{ \pm} \\
& \\
1 & 0
\end{array}\right), \quad \sigma^{ \pm}=\operatorname{tr} \widetilde{A}^{ \pm}<0, \quad \delta^{ \pm}=-\operatorname{det} \widetilde{A}^{ \pm}<0 .
$$

Demonstração: A hipótese de ser costura em $\Sigma-\{(0,0)\}$ implica que $\widetilde{a}_{21}^{-} \widetilde{a}_{21}^{+} x^{2}>0$, para $x \neq 0$. Portanto, $\widetilde{a}_{21}^{-} \widetilde{a}_{21}^{+}>0$. Em particular, $\widetilde{a}_{21}^{-} \neq 0$ e $\widetilde{a}_{21}^{+} \neq 0$. Seja $C^{ \pm}=\left(c_{i j}^{ \pm}\right)$a matriz de mudança de variáveis. A matriz $C^{ \pm}$preserva a reta de separação $\Sigma$ se e somente se $c_{11}^{ \pm}=1$ e $c_{21}^{ \pm}=0$. Assim defina a matriz

$$
C^{ \pm}=\left(\begin{array}{cc}
1 & \frac{\widetilde{a}_{22}^{ \pm}}{\widetilde{a}_{21}^{ \pm}} \\
0 & \frac{1}{\widetilde{a}_{21}^{ \pm}}
\end{array}\right)
$$

Por um cálculo simples, temos

$$
A^{ \pm}=C^{ \pm} \widetilde{A}^{ \pm}\left(C^{ \pm}\right)^{-1}=\left(\begin{array}{cc}
\widetilde{a}_{11}^{ \pm}+\widetilde{a}_{22}^{ \pm} & -\left(\widetilde{a}_{11}^{ \pm} \widetilde{a}_{22}^{ \pm}-\widetilde{a}_{12}^{ \pm} \widetilde{a}_{21}^{ \pm}\right) \\
1 & 0
\end{array}\right)=\left(\begin{array}{cc}
\sigma^{ \pm} & \delta^{ \pm} \\
& \\
1 & 0
\end{array}\right) .
$$

Veja que a função $H: \mathbb{R}^{2} \rightarrow \mathbb{R}^{2}$, definida por $H(X)=C^{+} X$, se $X \in \Sigma^{+} \cup \Sigma$ e $H(X)=C^{-} X$, se $X \in \Sigma^{-} \cup \Sigma$ é um homeomorfismo que estabelece uma equivalência topológica entre os sistemas (3.2) e (3.3). Além disso, os pontos em $\Sigma-\{(0,0)\}$ são do tipo costura para todo $\varepsilon$. De fato, seja $X_{0}=\left(x_{0}, 0\right) \in \Sigma-\{(0,0)\}$ e tomando $N=\nabla \mathcal{H}(X)=(0,1)$ obtemos

$$
\left(\left(A^{-} X_{0}\right) \cdot \nabla \mathcal{H}\left(X_{0}\right)\right)\left(\left(A^{+} X_{0}\right) \cdot \nabla \mathcal{H}\left(X_{0}\right)\right)=x_{0}^{2}>0
$$

onde as matrizes $A^{ \pm}$são dadas em (3.3). 
Observação 3.2.1. Tomando a mudança adicional de variáveis $x \mapsto x-\sigma^{ \pm} y$ e $y \mapsto y$ em cada zona, é simples provar que o sistema (3.2) torna-se um sistema contínuo, não só na origem, mas em todos os pontos da linha de separação. Ver Observação $3.3 \mathrm{em}$ [16].

O sistema (3.3), em cada zona, tem os seguintes equilíbrios para a origem:

i. Um nó atrator com autovalores distintos, denotado por N1;

ii. Um nó atrator não-diagonalizável, denotado por N2;

iii. Um foco atrator, denotado por FA.

Veja que o caso em que o equilíbrio é um nó atrator estrelado (um nó diagonalizável com autovalores iguais) a condição de costura em $\Sigma-\{(0,0)\}$ não é satisfeita.

As soluções de $\dot{X}=A^{-} X$ serão denotadas por

$$
\left(t, X_{0}\right) \mapsto X^{-}\left(t, X_{0}\right)=\left(x^{-}\left(t, X_{0}\right), y^{-}\left(t, X_{0}\right)\right),
$$

e as soluções de $\dot{X}=A^{+} X$ por

$$
\left(t, X_{0}\right) \mapsto X^{+}\left(t, X_{0}\right)=\left(x^{+}\left(t, X_{0}\right), y^{+}\left(t, X_{0}\right)\right)
$$

Nas próximas sub-seções faremos o estudo do sistema (3.3) somente para a matriz $A^{+}$. Resultados similares são obtidos para a matriz $A^{-}$.

\subsubsection{Caso 1. A origem é um nó atrator N1.}

Suponha que a origem é um nó atrator com autovalores distintos do sistema $\dot{X}=A^{+} X$ definido em $\Sigma^{+} \cup \Sigma$. Denotemos os autovalores de $A^{+}$por

$$
r_{1}^{+}=\frac{\sigma^{+}}{2}+\frac{\sqrt{\left(\sigma^{+}\right)^{2}+4 \delta^{+}}}{2}<0, \quad r_{2}^{+}=\frac{\sigma^{+}}{2}-\frac{\sqrt{\left(\sigma^{+}\right)^{2}+4 \delta^{+}}}{2}<0,
$$

isto é $r_{2}^{+}<r_{1}^{+}<0$. Autovetores associados para $r_{1}^{+}$e $r_{2}^{+}$são $v_{1}^{+}=\left(r_{1}^{+}, 1\right)$ e $v_{2}^{+}=\left(r_{2}^{+}, 1\right)$, respectivamente. Assim, a inclinação negativa de $v_{2}^{+}$é maior que a inclinação negativa de $v_{1}^{+}$. Defina os seguintes conjuntos

$$
\begin{gathered}
E_{1}^{+}=\left\{(x, y) \in \mathbb{R}^{2}: x<0, y=x / r_{1}^{+}\right\}, \\
E_{2}^{+}=\left\{(x, y) \in \mathbb{R}^{2}: x<0, y=x / r_{2}^{+}\right\}, \\
R_{1}^{+}=\left\{(x, y) \in \mathbb{R}^{2}: y>0, x>r_{1}^{+} y\right\}, \\
R_{2}^{+}=\left\{(x, y) \in \mathbb{R}^{2}: x<0, \frac{x}{r_{2}^{+}}<y<\frac{x}{r_{1}^{+}}\right\}, \\
R_{3}^{+}=\left\{(x, y) \in \mathbb{R}^{2}: y>0, x<r_{2}^{+} y\right\} .
\end{gathered}
$$

Além disso, temos que

$$
\Sigma^{+}=R_{1}^{+} \cup E_{1}^{+} \cup R_{2}^{+} \cup E_{2}^{+} \cup R_{3}^{+} .
$$


Lema 3.2.2. Considere $\dot{X}=A^{+} X$ definido em $\Sigma^{+} \cup \Sigma$ conforme (3.3) e as construções acima. As seguintes afirmações são verdadeiras (veja Figura 3.1):

1. Se $X_{0} \in E_{1}^{+} \cup E_{2}^{+}$, então $\lim _{t \rightarrow \infty} X^{+}\left(t, X_{0}\right)=(0,0)$;

2. Se $X_{0} \in R_{1}^{+}$, então

2.1. $\lim _{t \rightarrow \infty} X^{+}\left(t, X_{0}\right)=(0,0)$;

2.2. Existe $t_{X_{0}}<0$ tal que $X^{+}\left(t_{X_{0}}, X_{0}\right) \in \Sigma$;

3. Se $X_{0} \in R_{2}^{+}$, então $\lim _{t \rightarrow \infty} X^{+}\left(t, X_{0}\right)=(0,0)$;

4. Se $X_{0} \in R_{3}^{+}$, então existe $t_{X_{0}}>0$ tal que $X^{+}\left(t_{X_{0}}, X_{0}\right) \in \Sigma$.

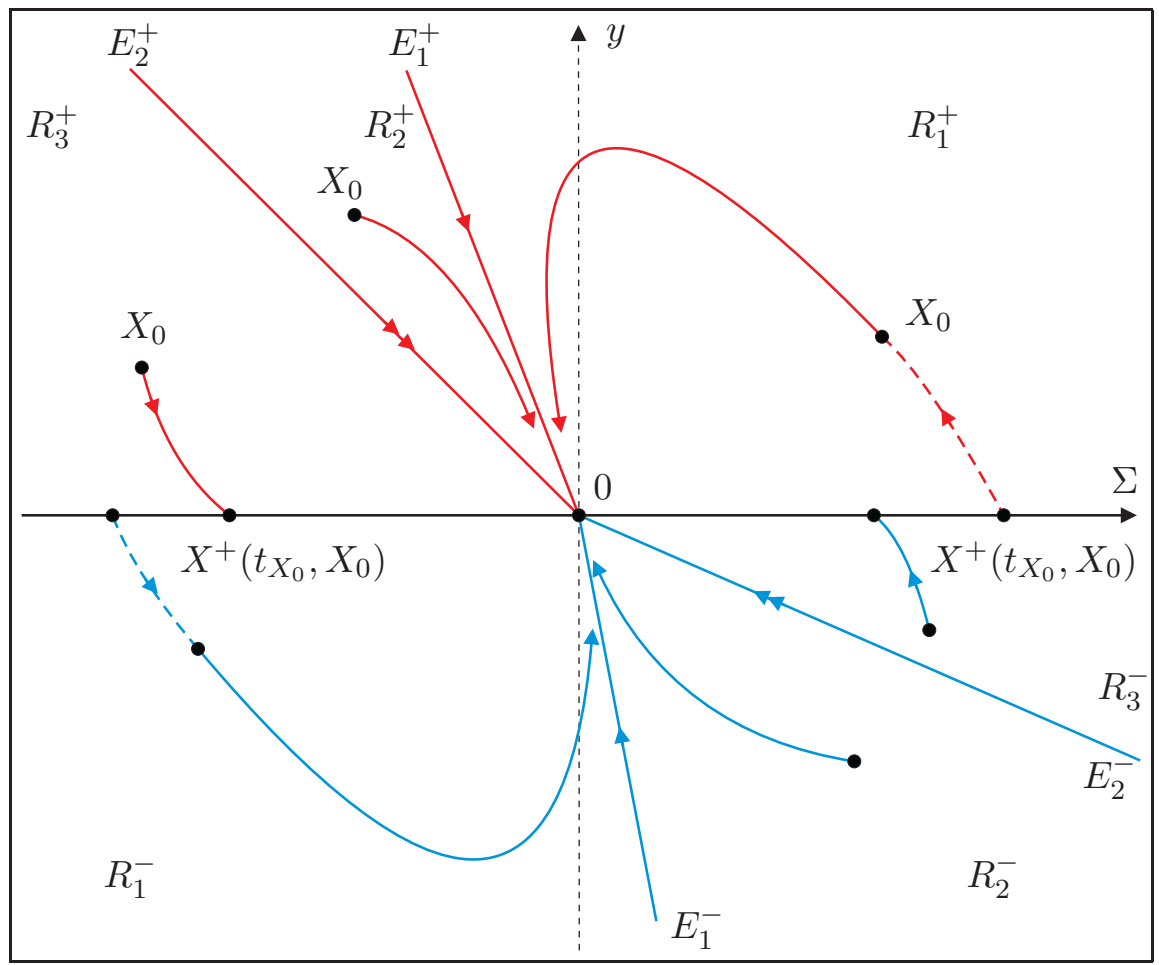

Figura 3.1: A origem é um nó atrator com autovalores distintos. As curvas em vermelho (azul) são as órbitas de $\dot{X}=A^{+} X\left(\dot{X}=A^{-} X\right)$.

Demonstração: As componentes da solução (3.5) são dadas por

$$
x^{+}\left(t, X_{0}\right)=e^{r_{1}^{+} t} r_{1}^{+}\left(\frac{x_{0}-y_{0} r_{2}^{+}}{r_{1}^{+}-r_{2}^{+}}\right)+e^{r_{2}^{+} t} r_{2}^{+}\left(\frac{y_{0} r_{1}^{+}-x_{0}}{r_{1}^{+}-r_{2}^{+}}\right)
$$

e

$$
y^{+}\left(t, X_{0}\right)=e^{r_{1}^{+} t}\left(\frac{x_{0}-y_{0} r_{2}^{+}}{r_{1}^{+}-r_{2}^{+}}\right)+e^{r_{2}^{+} t}\left(\frac{y_{0} r_{1}^{+}-x_{0}}{r_{1}^{+}-r_{2}^{+}}\right) .
$$

A prova dos itens 1, 2.1 e 3 segue da teoria clássica das EDO. Temos somente que provar os itens 2.2 e 4 . Para que $X^{+}\left(t, X_{0}\right) \in \Sigma$ devemos ter $y^{+}\left(t, X_{0}\right)=0$, a partir do qual resulta

$$
t_{X_{0}}=\frac{1}{r_{1}^{+}-r_{2}^{+}} \ln \left(\frac{x_{0}-y_{0} r_{1}^{+}}{x_{0}-y_{0} r_{2}^{+}}\right) \text {. }
$$


Por um cálculo simples obtemos

$$
0<\frac{x_{0}-y_{0} r_{1}^{+}}{x_{0}-y_{0} r_{2}^{+}}<1, \text { se } X_{0} \in R_{1}^{+} \text {e } \frac{x_{0}-y_{0} r_{1}^{+}}{x_{0}-y_{0} r_{2}^{+}}>1, \text { se } X_{0} \in R_{3}^{+} .
$$

Portanto, $t_{X_{0}}<0$ se $X_{0} \in R_{1}^{+}$e $t_{X_{0}}>0$ se $X_{0} \in R_{3}^{+}$.

\subsubsection{Caso 2. A origem é um nó atrator N2.}

Suponha que a origem é um nó atrator não-diagonalizável do sistema $\dot{X}=A^{+} X$ definido em $\Sigma^{+} \cup \Sigma$. Denote os autovalores repetidos $A^{+}$por

$$
r_{1}^{+}=r_{2}^{+}=\frac{\sigma^{+}}{2}<0
$$

Um autovetor associado a este autovalor é $v^{+}=\left(\sigma^{+} / 2,1\right)$. Assim, $v^{+}$tem inclinação negativa. Defina os seguintes conjuntos

$$
\begin{aligned}
E^{+} & =\left\{(x, y) \in \mathbb{R}^{2}: x<0, y=\frac{2 x}{\sigma^{+}}\right\}, \\
S_{1}^{+} & =\left\{(x, y) \in \mathbb{R}^{2}: y>0, x>\frac{\sigma^{+} y}{2}\right\}, \\
S_{2}^{+} & =\left\{(x, y) \in \mathbb{R}^{2}: y>0, x<\frac{\sigma^{+} y}{2}\right\} .
\end{aligned}
$$

Além disso, temos que

$$
\Sigma^{+}=S_{1}^{+} \cup E^{+} \cup S_{2}^{+}
$$

Lema 3.2.3. Considere $\dot{X}=A^{+} X$ definido em $\Sigma^{+} \cup \Sigma$ conforme (3.3) e as construções acima. As seguintes afirmações são verdadeiras (veja Figura 3.2):

1. Se $X_{0} \in E^{+}$, então $\lim _{t \rightarrow \infty} X^{+}\left(t, X_{0}\right)=(0,0)$;

2. Se $X_{0} \in S_{1}^{+}$, então

2.1. $\lim _{t \rightarrow \infty} X^{+}\left(t, X_{0}\right)=(0,0)$;

2.2. Existe $t_{X_{0}}<0$ tal que $X^{+}\left(t_{X_{0}}, X_{0}\right) \in \Sigma$;

3. Se $X_{0} \in S_{2}^{+}$, então existe $t_{X_{0}}>0$ tal que $X^{+}\left(t_{X_{0}}, X_{0}\right) \in \Sigma$.

Demonstração: As componentes da solução (3.5) são dadas por

$$
x^{+}\left(t, X_{0}\right)=e^{\frac{\sigma^{+}}{2} t}\left[x_{0}+\frac{\sigma^{+}}{2}\left(x_{0}-\frac{\sigma^{+}}{2} y_{0}\right) t\right]
$$

e

$$
y^{+}\left(t, X_{0}\right)=e^{\frac{\sigma^{+}}{2} t}\left[y_{0}+\left(x_{0}-\frac{\sigma^{+}}{2} y_{0}\right) t\right] .
$$




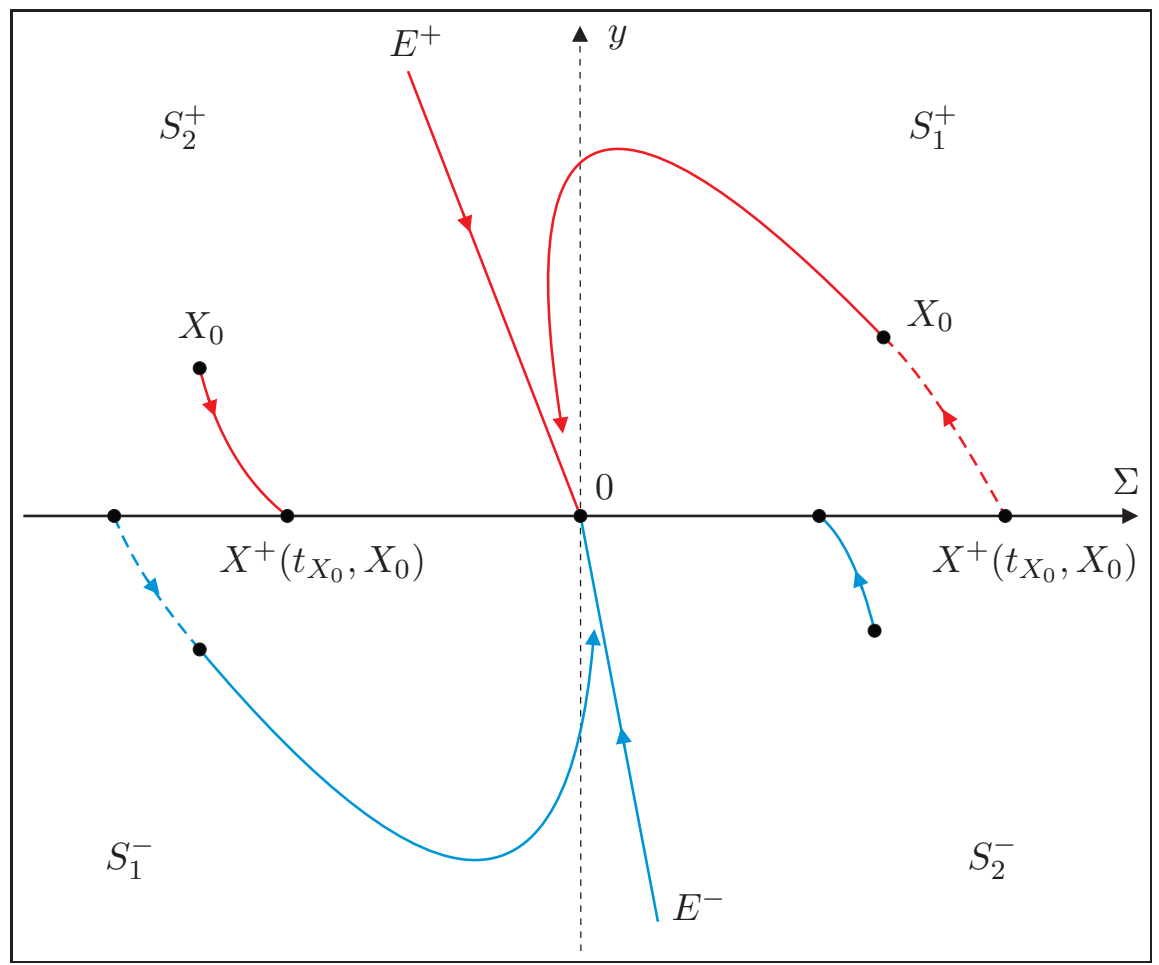

Figura 3.2: A origem é um nó atrator não-diagonalizável. As curvas em vermelho (azul) são as órbitas de $\dot{X}=A^{+} X\left(\dot{X}=A^{-} X\right)$.

A prova dos itens 1 e 2.1 segue da teoria clássica das EDO. Temos somente que provar os itens 2.2 e 3. Para que $X^{+}\left(t, X_{0}\right) \in \Sigma$ devemos ter $y^{+}\left(t, X_{0}\right)=0$, a partir do qual resulta

$$
t_{X_{0}}=\frac{y_{0}}{\frac{\sigma^{+}}{2} y_{0}-x_{0}}
$$

Portanto, $t_{X_{0}}<0$ se $X_{0} \in S_{1}^{+}$e $t_{X_{0}}>0$ se $X_{0} \in S_{2}^{+}$.

\subsubsection{Caso 3. A origem é um foco atrator FA.}

Suponha que a origem é um foco atrator do sistema $\dot{X}=A^{+} X$ definido em $\Sigma^{+} \cup \Sigma$. Provamos o seguinte lema. Veja Figura 3.3.

Lema 3.2.4. Considere $\dot{X}=A^{+} X$ definido em $\Sigma^{+} \cup \Sigma$ conforme (3.3). Suponha que a origem é um foco atrator. Dado $X_{0} \in \Sigma^{+}$, existem $t_{X_{0}}^{1}<0<t_{X_{0}}^{2}$ tal que $X^{+}\left(t_{X_{0}}^{1}, X_{0}\right) \in \Sigma$ e $X^{+}\left(t_{X_{0}}^{2}, X_{0}\right) \in \Sigma$.

Demonstração: Denotemos os autovalores de $A^{+}$por

$$
r_{1}^{+}=\lambda^{+}+i \mu^{+}, \quad r_{2}^{+}=\lambda^{+}-i \mu^{+},
$$




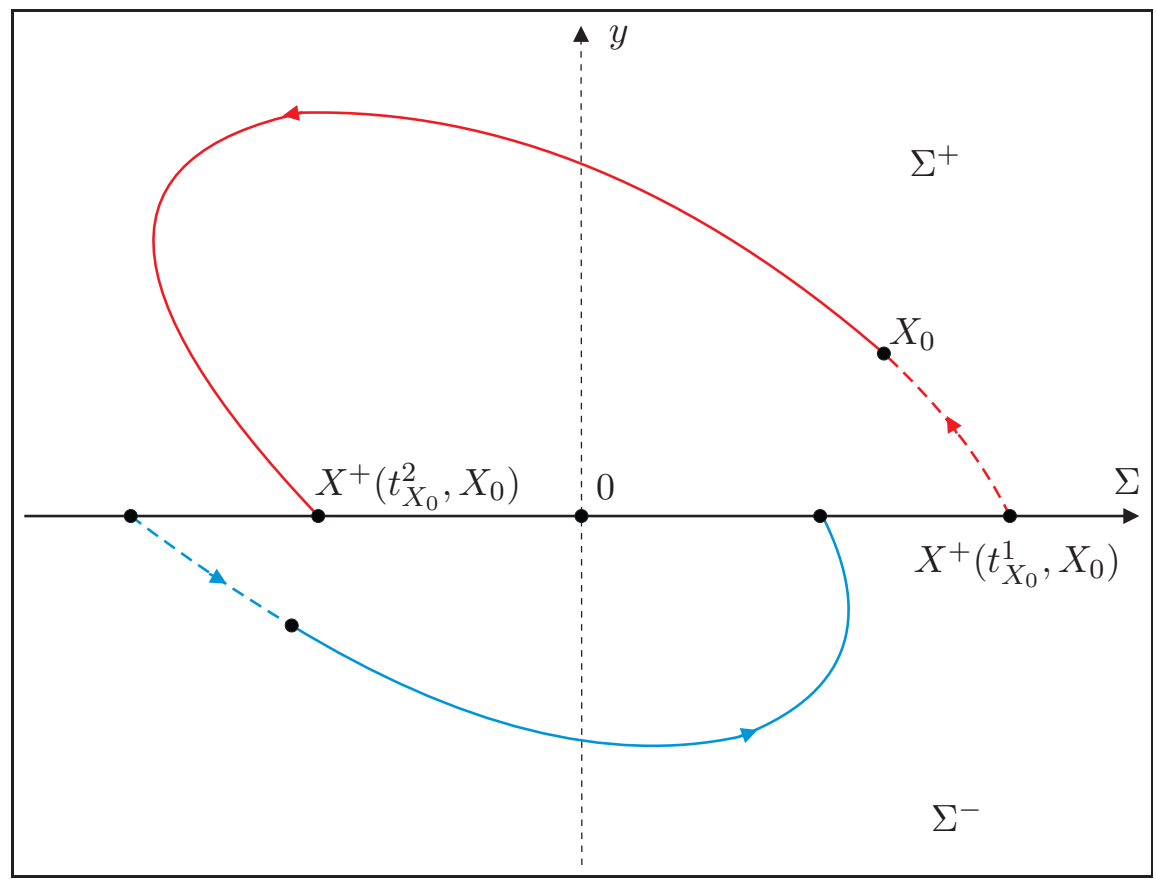

Figura 3.3: A origem é um foco atrator. As curvas em vermelho (azul) são as órbitas de $\dot{X}=A^{+} X$ $\left(\dot{X}=A^{-} X\right)$.

onde

$$
\lambda^{+}=\frac{\sigma^{+}}{2}<0, \quad \mu^{+}=\frac{\sqrt{-4 \delta^{+}-\left(\sigma^{+}\right)^{2}}}{2}>0 .
$$

As componentes da solução (3.5) são dadas por

$$
x^{+}\left(t, X_{0}\right)=e^{\lambda^{+} t}\left[x_{0} \cos \left(\mu^{+} t\right)+\left(\frac{\lambda^{+}}{\mu^{+}}\left(x_{0}-y_{0} \lambda^{+}\right)-\mu^{+} y_{0}\right) \operatorname{sen}\left(\mu^{+} t\right)\right]
$$

$\mathrm{e}$

$$
y^{+}\left(t, X_{0}\right)=e^{\lambda^{+} t}\left[y_{0} \cos \left(\mu^{+} t\right)+\left(\frac{x_{0}-y_{0} \lambda^{+}}{\mu^{+}}\right) \operatorname{sen}\left(\mu^{+} t\right)\right] .
$$

Defina o conjunto

$$
W^{+}=\left\{(x, y) \in \mathbb{R}^{2}: y>0, x=\lambda^{+} y\right\}
$$

Se $X_{0} \in W^{+}$então $y^{+}\left(t, X_{0}\right)=0$ se, e somente se,

$$
t_{X_{0}}^{1}=-\frac{\pi}{2 \mu^{+}}<0, \quad t_{X_{0}}^{2}=\frac{\pi}{2 \mu^{+}}>0 .
$$

Assim $X^{+}\left(t_{X_{0}}^{1}, X_{0}\right) \in \Sigma$ e $X^{+}\left(t_{X_{0}}^{2}, X_{0}\right) \in \Sigma$. Agora, é suficiente mostrar que para $X_{0} \in \Sigma^{+}-W^{+}$ existe um tempo $t_{X_{0}} \in \mathbb{R}$ tal que $X^{+}\left(t_{X_{0}}, X_{0}\right) \in W^{+}$, isto é, $x^{+}\left(t_{X_{0}}, X_{0}\right)=\lambda^{+} y^{+}\left(t_{X_{0}}, X_{0}\right)$. Esta última igualdade acontece se, e somente se,

$$
t_{X_{0}}=\frac{1}{\mu^{+}} \arctan \left(\frac{x_{0}-\lambda^{+} y_{0}}{\mu^{+} y_{0}}\right)
$$

onde $t_{X_{0}} \in\left(0, \pi / 2 \mu^{+}\right)$se $x_{0}-\lambda^{+} y_{0}>0$ e $t_{X_{0}} \in\left(-\pi / 2 \mu^{+}, 0\right)$ se $x_{0}-\lambda^{+} y_{0}<0$. 
Proposição 3.2.1. Considere o sistema (3.3). Suponha que a origem é um nó atrator N1 para $\dot{X}=A^{+} X$ e $\dot{X}=A^{-} X$. Então a origem é globalmente assintoticamente estável.

Demonstração: A prova segue imediatamente do Lema 3.2.2. Veja a Figura 3.1.

Proposição 3.2.2. Considere o sistema (3.3). Suponha que a origem é um nó atrator N1 para o sistema $\dot{X}=A^{+} X$ e um nó atrator N2 para $\dot{X}=A^{-} X$. Então a origem é globalmente assintoticamente estável.

Demonstração: A prova segue imediatamente dos Lemas 3.2.2 e 3.2.3. Veja a Figura 3.1 (em vermelho) e a Figura 3.2 (em azul).

Proposição 3.2.3. Considere o sistema (3.3). Suponha que a origem é um nó atrator N1 para $\dot{X}=A^{+} X$ e um foco atrator FA para $\dot{X}=A^{-} X$. Então a origem é globalmente assintoticamente estável.

Demonstração: A prova segue imediatamente dos Lemas 3.2.2 e 3.2.4. Veja a Figura 3.1 (em vermelho) e a Figura 3.3 (em azul).

Proposição 3.2.4. Considere o sistema (3.3). Suponha que a origem é um nó atrator N2 para $\dot{X}=A^{+} X$ e $\dot{X}=A^{-} X$. Então a origem é globalmente assintoticamente estável.

Demonstração: A prova segue imediatamente do Lema 3.2.3, Veja a Figura 3.2,

Proposição 3.2.5. Considere o sistema (3.3). Suponha que a origem é um nó atrator N2 para $\dot{X}=A^{+} X$ e um foco atrator FA para $\dot{X}=A^{-} X$. Então a origem é globalmente assintoticamente estável.

Demonstração: A prova segue imediatamente dos Lemas 3.2.3 e 3.2.4. Veja a Figura 3.2 (em vermelho) e a Figura 3.3 (em azul).

Proposição 3.2.6. Considere o sistema (3.3). Suponha que a origem é um foco atrator FA para $\dot{X}=A^{+} X$ e $\dot{X}=A^{-} X$. Então a origem é globalmente assintoticamente estável.

Demonstração: Do Lema 3.2.4, está bem definida em

$$
\Sigma_{>0}=\{(x, 0) \in \Sigma: x>0\}
$$

a aplicação de Poincaré

$$
\Pi: \Sigma_{>0} \rightarrow \Sigma_{>0}, \quad x_{0} \mapsto \Pi\left(x_{0}\right)=x^{-}\left(\frac{\pi}{\mu^{-}}, x^{+}\left(\frac{\pi}{\mu^{+}},\left(x_{0}, 0\right)\right)\right),
$$


onde

$$
\begin{aligned}
& x^{+}\left(t, X_{0}\right)=e^{\lambda^{+} t}\left[x_{0} \cos \left(\mu^{+} t\right)+\left(\frac{\lambda^{+}}{\mu^{+}}\left(x_{0}-y_{0} \lambda^{+}\right)-\mu^{+} y_{0}\right) \operatorname{sen}\left(\mu^{+} t\right)\right], \\
& x^{-}\left(t, X_{0}\right)=e^{\lambda^{-} t}\left[x_{0} \cos \left(\mu^{-} t\right)+\left(\frac{\lambda^{-}}{\mu^{-}}\left(x_{0}-y_{0} \lambda^{-}\right)-\mu^{-} y_{0}\right) \operatorname{sen}\left(\mu^{-} t\right)\right],
\end{aligned}
$$

e

$$
r_{1}^{+}=\lambda^{+}+i \mu^{+}, \quad r_{2}^{+}=\lambda^{+}-i \mu^{+}, \quad r_{1}^{-}=\lambda^{-}+i \mu^{-}, \quad r_{2}^{-}=\lambda^{-}-i \mu^{-},
$$

com

$$
\begin{aligned}
& \lambda^{+}=\frac{\sigma^{+}}{2}<0, \quad \mu^{+}=\frac{\sqrt{-4 \delta^{+}-\left(\sigma^{+}\right)^{2}}}{2}>0, \\
& \lambda^{-}=\frac{\sigma^{-}}{2}<0, \quad \mu^{-}=\frac{\sqrt{-4 \delta^{-}-\left(\sigma^{-}\right)^{2}}}{2}>0,
\end{aligned}
$$

os autovalores de $A^{+}$e $A^{-}$, respectivamente.

Efetuando os cálculos, obtemos

$$
\Pi\left(x_{0}\right)=e^{\left(\frac{\lambda^{+}}{\mu^{+}}+\frac{\lambda^{-}}{\mu^{-}}\right) \pi} x_{0}
$$

Como

$$
\frac{\lambda^{+}}{\mu^{+}}+\frac{\lambda^{-}}{\mu^{-}}<0
$$

segue que $\Pi\left(x_{0}\right)<x_{0}$, para todo $x_{0} \in \Sigma_{>0}$ e isto implica que a aplicação de Poincaré é uma contração.

A prova do Teorema 3.1.1 segue das proposições 3.2.1, 3.2.2, 3.2.3, 3.2.4, 3.2.5 e 3.2.6.

\subsection{Conjunto de Separação é uma Curva Poligonal}

\section{Prova do Teorema 3.1 .2}

Considere

$$
\dot{X}=\left\{\begin{array}{cc}
A^{-} X, & \mathcal{H}_{\rho}(X) \leq 0 \\
A^{+} X, & \mathcal{H}_{\rho}(X) \geq 0
\end{array}\right.
$$

com

$$
A^{-}=\left(\begin{array}{cc}
2 \lambda^{-} & -\left(\left(\lambda^{-}\right)^{2}+\left(\mu^{-}\right)^{2}\right) \\
1 & 0
\end{array}\right), \quad A^{+}=\left(\begin{array}{cc}
\lambda^{+} & -\mu^{+} \\
\mu^{+} & \lambda^{+}
\end{array}\right)
$$

onde $\lambda^{ \pm}<0$ e $\mu^{ \pm}>0$ e a função $\mathcal{H}_{\rho}$ é definida por

$$
X \mapsto \mathcal{H}_{\rho}(X)=y-\psi_{\rho}(x)
$$




$$
x \mapsto \psi_{\rho}(x)= \begin{cases}0, & x \leq 0, \quad \rho \geq 0 . \\ \rho x, & x \geq 0,\end{cases}
$$

Portanto, a curva de separação

$$
\Sigma_{\rho}=\left\{X \in \mathbb{R}^{2}: \mathcal{H}_{\rho}=0\right\}
$$

é uma curva poligonal para cada $\rho>0$. Veja a Figura 3.4.

Denote por $\left(A^{-}, A^{+}, \Sigma_{\rho}\right)$ a família de sistemas diferenciais por partes planares com duas zonas definida por (3.6) e (3.7) indexada pelo parâmetro $\rho$.

Proposição 3.3.1. As seguintes afirmações são verdadeiras:

a. Existe um intervalo não vazio da forma $I_{0}=[0, a)$ tal que a origem é ponto de equlíbrio globalmente assintoticamente estável de $\left(A^{-}, A^{+}, \Sigma_{\rho}\right)$, para todo $\rho \in I_{0}$;

b. Existe um valor $\rho=\rho_{c}$ tal que a origem é um centro de $\left(A^{-}, A^{+}, \Sigma_{\rho_{c}}\right)$;

c. Existe um intervalo da forma $I_{\infty}=[b, \infty)$ tal que a origem é ponto de equlíbrio instável de $\left(A^{-}, A^{+}, \Sigma_{\rho}\right)$, para todo $\rho \in I_{\infty}$.

Além disso, os pontos em $\Sigma_{\rho}-\{(0,0)\}$ são do tipo costura, para todo $\rho \geq 0$.

Demonstração: Primeiramente vamos estudar os pontos em $\Sigma_{\rho}-\{(0,0)\}$. Para $x \neq 0$ obtemos

$$
\left\{\begin{aligned}
\mu^{+} x^{2}>0, & (x, y)=(x, 0) \\
\left(1+\rho^{2}\right)\left(\left(1-\lambda^{-} \rho\right)^{2}+\left(\mu^{-}\right)^{2} \rho^{2}\right) \mu^{+} x^{2}>0, & (x, y)=(x, \rho x)
\end{aligned}\right.
$$

e assim segue que os pontos em $\Sigma_{\rho}-\{(0,0)\}$ são do tipo costura.

Agora defina $\eta^{ \pm}=\lambda^{ \pm} / \mu^{ \pm}$. A fim de determinar a estabilidade da origem, vamos estudar as propriedades da função de separação $x_{0} \mapsto \delta\left(x_{0}\right)$ associada a um membro da família $\left(A^{-}, A^{+}, \Sigma_{\rho}\right)$ e construída a partir dos componentes das soluções (3.4) e (3.5) dadas por

$$
\begin{aligned}
& x^{-}\left(t, X_{0}\right)=e^{\lambda^{-} t}\left[x_{0} \cos \left(\mu^{-} t\right)+\left(\eta^{-}\left(x_{0}-y_{0} \lambda^{-}\right)-\mu^{-} y_{0}\right) \operatorname{sen}\left(\mu^{-} t\right)\right], \\
& y^{-}\left(t, X_{0}\right)=e^{\lambda^{-} t}\left[\frac{1}{\mu^{-}} \operatorname{sen}\left(\mu^{-} t\right) x_{0}+\left(\cos \left(\mu^{-} t\right)-\eta^{-} \operatorname{sen}\left(\mu^{-} t\right)\right) y_{0}\right], \\
& x^{+}\left(t, X_{0}\right)=e^{\lambda^{+} t}\left[\cos \left(\mu^{+} t\right) x_{0}-\operatorname{sen}\left(\mu^{+} t\right) y_{0}\right], \\
& y^{+}\left(t, X_{0}\right)=e^{\lambda^{+} t}\left[\operatorname{sen}\left(\mu^{+} t\right) x_{0}+\cos \left(\mu^{+} t\right) y_{0}\right] .
\end{aligned}
$$

Seja $X_{0}=\left(x_{0}, y_{0}\right)=\left(x_{0}, \psi_{\rho}\left(x_{0}\right)\right)$ qualquer condição inicial com $x_{0}>0$. A função de separação é definida por

$$
x_{0} \mapsto \delta\left(x_{0}\right)=x^{-}\left(-\tau^{-}, X_{0}\right)-x^{+}\left(\tau^{+}, X_{0}\right),
$$




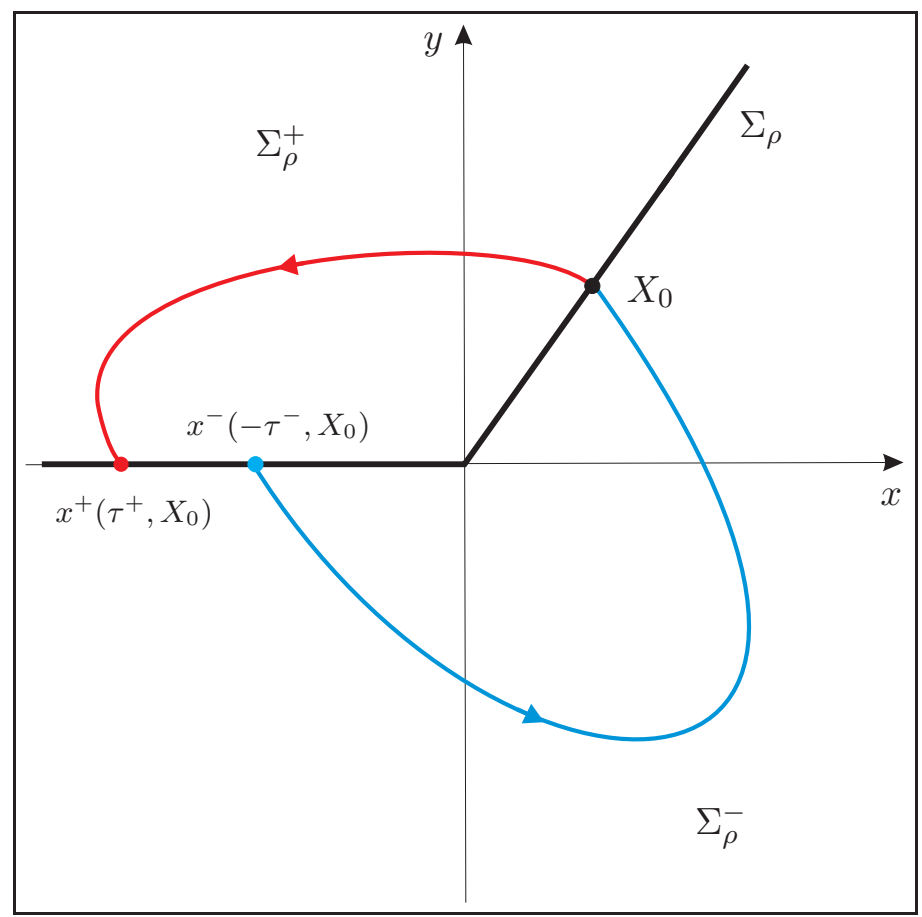

Figura 3.4: Função de separação $\delta=x^{-}-x^{+}$, com $\delta>0$, onde $\Sigma_{\rho}^{+}=\left\{(x, y) \in \mathbb{R}^{2}: y>\psi_{\rho}(x)\right\}$ e $\Sigma_{\rho}^{-}=\left\{(x, y) \in \mathbb{R}^{2}: y<\psi_{\rho}(x)\right\}$.

onde $\tau^{-}>0$ é o menor tempo tal que $X^{-}\left(-\tau^{-}, X_{0}\right) \in \Sigma_{\rho}$ e $\tau^{+}>0$ é o menor tempo tal que $X^{+}\left(\tau^{+}, X_{0}\right) \in \Sigma_{\rho}$. Veja Figura 3.4.

O tempo $\tau^{-}$é a solução da equação $y^{-}\left(-\tau^{-}, X_{0}\right)=0$ e $\tau^{+}$é a solução de $y^{+}\left(\tau^{+}, X_{0}\right)=0$. Assim,

$$
\begin{aligned}
& \tau^{-}=\tau^{-}\left(x_{0}\right)=\frac{1}{\mu^{-}}\left(\pi+\arctan \left(\frac{\mu^{-} \psi_{\rho}\left(x_{0}\right)}{x_{0}-\lambda^{-} \psi_{\rho}\left(x_{0}\right)}\right)\right), \\
& \tau^{+}=\tau^{+}\left(x_{0}\right)=\frac{1}{\mu^{+}}\left(\pi-\arctan \left(\frac{\psi_{\rho}\left(x_{0}\right)}{x_{0}}\right)\right) .
\end{aligned}
$$

Substituindo os tempos (3.10) em (3.8), segue que

$$
\begin{aligned}
x^{-}\left(-\tau^{-}, X_{0}\right) & =-e^{-\lambda^{-} \tau^{-}} \sqrt{\left(x_{0}-\lambda^{-} \psi_{\rho}\left(x_{0}\right)\right)^{2}+\left(\mu^{-}\right)^{2} \psi_{\rho}\left(x_{0}\right)^{2}}, \\
x^{+}\left(\tau^{+}, X_{0}\right) & =-e^{\lambda^{+} \tau^{+}} \sqrt{x_{0}^{2}+\psi_{\rho}\left(x_{0}\right)^{2}} .
\end{aligned}
$$

Uma vez que $\psi_{\rho}\left(x_{0}\right)=\rho x_{0}$ para $x_{0}>0$, conforme (3.10)

$$
\begin{aligned}
& \tau^{-}\left(x_{0}\right)=\frac{1}{\mu^{-}}\left(\pi+\arctan \left(\frac{\mu^{-} \rho}{1-\lambda^{-} \rho}\right)\right), \\
& \tau^{+}\left(x_{0}\right)=\frac{1}{\mu^{+}}(\pi-\arctan (\rho)),
\end{aligned}
$$

e mantendo a mesma notação anterior para simplicidade, $\tau^{-}=\tau^{-}(\rho)$ e $\tau^{+}=\tau^{+}(\rho)$, (3.9) pode ser escrito a partir de (3.11) como

$$
x_{0} \mapsto \delta\left(x_{0}\right)=g(\rho) \Delta(\rho) x_{0},
$$


onde

$$
\begin{aligned}
& g(\rho)=e^{-\lambda^{-} \tau^{-}(\rho)+\varphi(\rho)} \sqrt{1+\rho^{2}}, \\
& \Delta(\rho)=e^{h(\rho)}-1, \\
& h(\rho)=\lambda^{+} \tau^{+}(\rho)+\lambda^{-} \tau^{-}(\rho)-\varphi(\rho), \\
& \varphi(\rho)=\frac{1}{2} \ln \left(\frac{\left(1-\lambda^{-} \rho\right)^{2}+\left(\mu^{-}\right)^{2} \rho^{2}}{1+\rho^{2}}\right),
\end{aligned}
$$

para $\rho \in[0, \infty)$.

A estabilidade da origem de $\left(A^{-}, A^{+}, \Sigma_{\rho}\right)$ depende somente da função $\Delta$ em (3.13) ou, mais precisamente, da função $h$ que tem as seguintes propriedades:

P1. $h \in \mathcal{C}^{\infty}([0, \infty), \mathbb{R})$;

P2. $h(0)=\pi\left(\eta^{+}+\eta^{-}\right)<0$;

P3. $h(\infty)=\lim _{\rho \rightarrow \infty} h(\rho)=\frac{\pi}{2} \eta^{+}+\pi \eta^{-}-\eta^{-} \arctan \left(\frac{1}{\eta^{-}}\right)-\frac{1}{2} \ln \left(\left(\lambda^{-}\right)^{2}+\left(\mu^{-}\right)^{2}\right)$.

Se existem valores $\lambda^{ \pm}<0$ e $\mu^{ \pm}>0$ tal que $h(\infty)>0$, então:

a. pelas propriedades $\mathbf{P} 1$ e $\mathbf{P} 2$ existe um intervalo não vazio da forma $I_{0}=[0, a)$ na qual $h$ mantém o sinal negativo para todo $\rho \in I_{0}$. Assim, se a condição inicial é $X_{0}=\left(x_{0}, y_{0}\right)=$ $\left(x_{0}, \psi_{\rho}\left(x_{0}\right)\right)$, onde $\psi_{\rho}\left(x_{0}\right)=\rho x_{0} \operatorname{com} x_{0}>0$ e $\rho \in I_{0}$, em seguida, segue-se a partir de (3.12) que $\delta\left(x_{0}\right)<0$ e a origem é um atrator global (um foco estável). O caso $\rho=0$ foi tratado na Proposição 3.2.6.

b. pelas propriedades $\mathbf{P 1}, \mathbf{P 2}$ e P3, e o Teorema de Bolzano, existe um valor $\rho=\rho_{c}>0$ tal que $h\left(\rho_{c}\right)=0$ e, consequentemente, $\delta\left(x_{0}\right) \equiv 0$ para toda condição inicial $X_{0}=\left(x_{0}, y_{0}\right)=$ $\left(x_{0}, \psi_{\rho}\left(x_{0}\right)\right)$ com $\psi_{\rho}\left(x_{0}\right)=\rho_{c} x_{0}$ e $x_{0}>0$. Portanto, neste caso a origem é um centro;

c. novamente pela continuidade da função $h$ e pela propriedade $\mathbf{P 3}$, existe um intervalo da forma $I_{\infty}=[b, \infty)$ na qual a função $h$ mantém o sinal positivo para todo $\rho \in I_{\infty}$. Usando argumento similar à do item a, a origem é agora um repulsor global (um foco instável).

A prova do Teorema 3.1 .2 estará completa se mostrarmos a existência de uma região no espaço de paramêtros $\lambda^{-} \mu^{-} \eta^{+}$que torna verdadeira a afirmação $h(\infty)>0$. Para isto, considere a função definida a partir da propriedade $\mathbf{P 3}$,

$$
\left(\lambda^{-}, \mu^{-}\right) \in D \mapsto G\left(\lambda^{-}, \mu^{-}\right),
$$

onde

$$
G\left(\lambda^{-}, \mu^{-}\right)=\frac{2}{\pi}\left(-\pi \frac{\lambda^{-}}{\mu^{-}}+\frac{\lambda^{-}}{\mu^{-}} \arctan \left(\frac{\mu^{-}}{\lambda^{-}}\right)+\frac{1}{2} \ln \left(\left(\lambda^{-}\right)^{2}+\left(\mu^{-}\right)^{2}\right)\right),
$$


e $D=\left\{\left(\lambda^{-}, \mu^{-}\right) \in \mathbb{R}^{2}: \lambda^{-}<0, \mu^{-}>0\right\}$. Se escolhermos $\eta^{+}$satisfazendo $G\left(\lambda^{-}, \mu^{-}\right)<\eta^{+}<0$ segue que $h(\infty)>0$. Entretanto, $G\left(\lambda^{-}, \mu^{-}\right)<0$ para todo par $\left(\lambda^{-}, \mu^{-}\right)$no interior da região $R \subset D$ cuja fronteira $\partial R$ é o traço da curva definida por partes

$$
\begin{aligned}
\alpha:[0, \pi] & \longrightarrow D \\
u & \longmapsto \alpha(u)=\left\{\begin{array}{l}
\alpha_{1}(u), \quad 0 \leq u<\frac{\pi}{2} \\
\alpha_{2}(u), \frac{\pi}{2} \leq u \leq \pi
\end{array}\right.
\end{aligned}
$$

onde

$$
\begin{aligned}
& \alpha_{1}(u)=\left(-e^{-(\pi+\arctan (\cot (u))) \tan (u)} \operatorname{sen}(u), e^{-(\pi+\arctan (\cot (u))) \tan (u)} \cos (u)\right), \\
& \alpha_{2}(u)=\left(0, \frac{2}{\pi}\left(u-\frac{\pi}{2}\right)\right) .
\end{aligned}
$$

Deve-se notar que $u \mapsto \alpha_{1}(u)$ segue de $G\left(\lambda^{-}, \mu^{-}\right)=0$ das parametrizações

$$
\lambda^{-}(u)=r(u) \cos \left(u+\frac{\pi}{2}\right), \mu^{-}(u)=r(u) \operatorname{sen}\left(u+\frac{\pi}{2}\right), u \in\left[0, \frac{\pi}{2}\right)
$$

e $u \mapsto \alpha_{2}(u)$ da restrição $\lambda^{-}<0$. A figura 3.5 mostra a região $R$ e a fronteira $\partial R$. Isto completa a prova do Teorema 3.1 .2 .

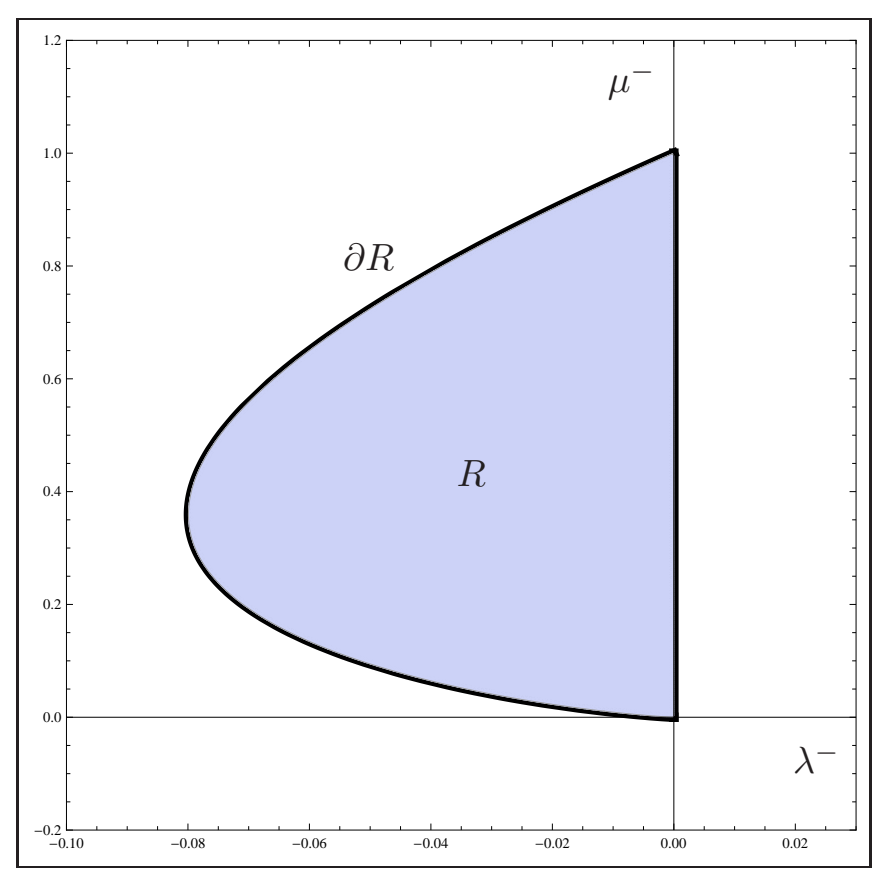

Figura 3.5: A região $R$ é ilustrada e preenchida de azul e a fronteira $\partial R$ é ilustrada pela linha preta grossa.

Para concluir esta seção apresentamos um exemplo do sistema $\left(A^{-}, A^{+}, \Sigma_{\rho}\right)$ na qual a origem é um foco instável. Como $G(-0.01,0.5)=-0.381399$ com seis decimais, o par $\left(\lambda^{-}, \mu^{-}\right)=$ $(-0.01,0.5) \in R$. Então escolhemos $\lambda^{+}=-0.2$ and $\mu^{+}=1$ e de acordo com o Teorema 3.1 .2 item c, para $\rho>0$ suficientemente grande, a origem é um foco instável. Neste caso, 
tomando $\rho=6$ segue que $h(\rho)>0$ e a Figura 3.6 mostra a trajetória através da condição inicial $X_{0}=\left(x_{0}, y_{0}\right)=(0.5,3)$.

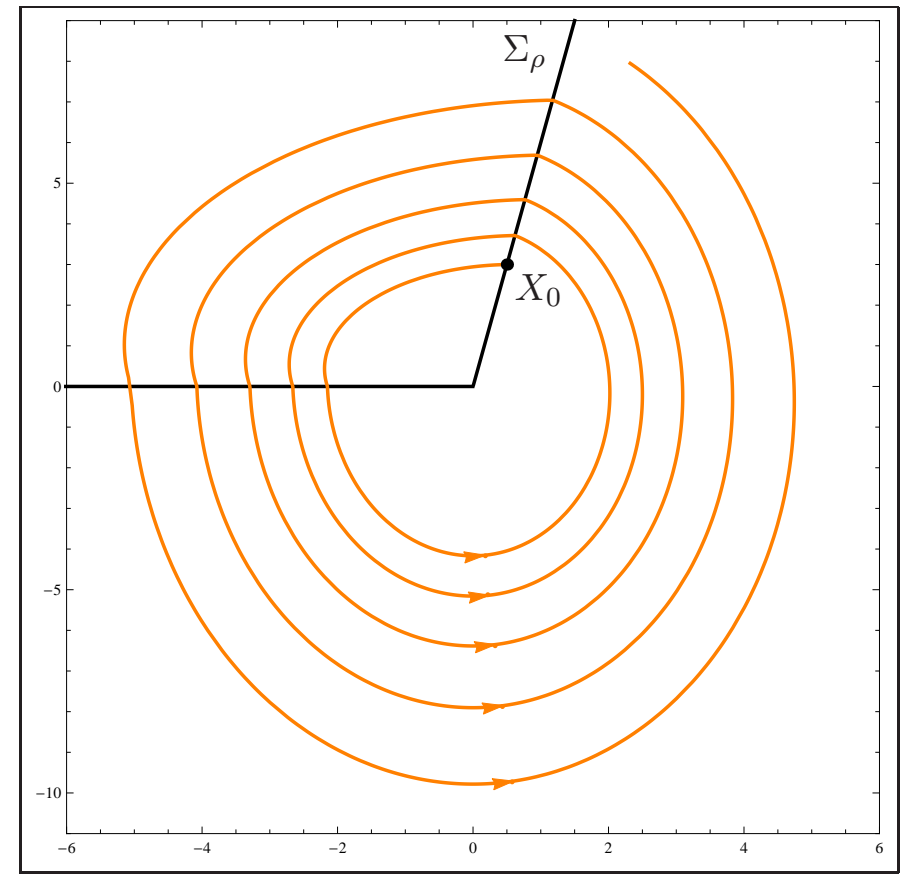

Figura 3.6: A origem de $\left(A^{-}, A^{+}, \Sigma_{\rho}\right)$ é um foco instável quando $\rho=6, \lambda^{-}=-0.01, \mu^{-}=0.5, \lambda^{+}=-0.2$ e $\mu^{+}=1$. A condição inicial ilustrada pelo ponto preto é $X_{0}=\left(x_{0}, y_{0}\right)=(0.5,3)$. 


\section{Capítulo 4}

\section{Continuação de Órbitas Periódicas e}

\section{Regularização de Sistemas}

\section{Descontínuos}

Neste capítulo, vamos estudar ciclos limites em sistemas suaves por partes no plano. Nos casos onde temos um centro linear ou um centro Hamiltoniano ambos com perturbações suaves por partes, usamos o método de regularização e damos uma prova para o uso de funções de Melnikov diretamente no problema não-suave original. Na verdade, estudamos a equivalência de ambos os métodos nestes dois casos. As principais referências deste capítulo são [1], [5], [11, 24] e [38].

\subsection{Centro Linear Perturbado por Partes}

Considere o sistema

$$
\dot{X}=\frac{d X}{d t}=Z(X, \varepsilon)= \begin{cases}X^{-}(X)=A X+\varepsilon G^{-}(X), & y \leq 0 \\ X^{+}(X)=A X+\varepsilon G^{+}(X), & y \geq 0\end{cases}
$$

com $X=(x, y) \in \mathbb{R}^{2}, \varepsilon \geq 0$ um parâmetro real suficientemente pequeno, o ponto denotando a derivada com respeito ao parâmetro $t$, chamada aqui de tempo,

$$
A=\left(\begin{array}{cc}
0 & -1 \\
1 & 0
\end{array}\right)
$$

$\mathrm{e}$

$$
\begin{aligned}
G: & \mathbb{R}^{2} \quad \longrightarrow \mathbb{R}^{2} \\
X & \longmapsto G(X)= \begin{cases}G^{-}(X), & y \leq 0, \\
G^{+}(X), & y \geq 0,\end{cases}
\end{aligned}
$$


sendo $G^{-}(x, y)=\left(g_{1}^{-}(x, y), g_{2}^{-}(x, y)\right)$ e $G^{+}(x, y)=\left(g_{1}^{+}(x, y), g_{2}^{+}(x, y)\right)$ campos vetoriais suaves (classe $\left.C^{k}, k \geq 1\right)$ e $G^{ \pm}(0,0)=(0,0)$.

Primeiramente, estudamos ciclos limites do sistema descontínuo (4.1) usando um tipo de função de Melnikov dada no seguinte teorema.

Teorema 4.1.1. Se a $>0$ é um zero simples da função

$$
\mathcal{M}(a)=\mathcal{M}^{+}(a)+\mathcal{M}^{-}(a)
$$

onde

$$
\mathcal{M}^{+}(a)=\int_{0}^{\pi}\left[g_{1}^{+}(a \cos (s), a \operatorname{sen}(s)) \cos (s)+g_{2}^{+}(a \cos (s), a \operatorname{sen}(s)) \operatorname{sen}(s)\right] \mathrm{d} s
$$

e

$$
\mathcal{M}^{-}(a)=\int_{\pi}^{2 \pi}\left[g_{1}^{-}(a \cos (s), a \operatorname{sen}(s)) \cos (s)+g_{2}^{-}(a \cos (s), a \operatorname{sen}(s)) \operatorname{sen}(s)\right] \mathrm{d} s,
$$

então para $\varepsilon>0$ suficientemente pequeno existe um ciclo limite $X^{\varepsilon}$ de (4.1) tal que $X^{\varepsilon}$ tende ao círculo de centro na origem e raio a quando e tende a 0 . O ciclo limite é estável se $\mathcal{M}^{\prime}(a)<0$ e instável se $\mathcal{M}^{\prime}(a)>0$.

Uma outra abordagem para o estudo das soluções periódicas do sistema descontínuo (4.1) baseia-se no método de regularização que descrevemos brevemente agora. Para mais detalhes veja a Seção 2.2 e suas referências.

Considere $\mathcal{H}: \mathbb{R}^{2} \rightarrow \mathbb{R}, \mathcal{H}(x, y)=y$. Veja que zero é um valor regular da função suave $\mathcal{H}$. Defina os conjuntos

$$
\begin{gathered}
\Sigma=\mathcal{H}^{-1}(0)=\left\{(x, y) \in \mathbb{R}^{2}: y=0\right\} \\
\Sigma^{-}=\mathcal{H}^{-1}(-\infty, 0)=\left\{(x, y) \in \mathbb{R}^{2}: y<0\right\}, \quad \Sigma^{+}=\mathcal{H}^{-1}(0,+\infty)=\left\{(x, y) \in \mathbb{R}^{2}: y>0\right\} .
\end{gathered}
$$

Então $\mathbb{R}^{2}=\Sigma^{-} \cup \Sigma \cup \Sigma^{+}$. O conjunto $\Sigma$, neste caso, é uma reta de separação entre as duas zonas $\Sigma^{-}$e $\Sigma^{+}$.

A função de transição $\varphi: \mathbb{R} \rightarrow \mathbb{R}, C^{k}, k \geq 1$, é definida por $\varphi(t)=0$, se $t \leq-1, \varphi(t)=1$, se $t \geq 1$ e $\varphi^{\prime}(t)>0$, se $t \in(-1,1)$. Além disso, definimos $\varphi^{\mu}(t)=\varphi(t / \mu)$, para todo $t \in \mathbb{R}$ e um número real $\mu>0$, chamado de parâmetro de regularização. Chamamos $\varphi^{\mu}$ de função de regularização.

A regularização do campo $Z$ em (4.1) é dada pela família a dois parâmetros de campos vetoriais suaves

$$
Z^{\mu}(X, \varepsilon)=\left(1-\varphi^{\mu}(y)\right) X^{-}(x, y)+\varphi^{\mu}(y) X^{+}(x, y)=A X+\varepsilon R(X, \mu),
$$

onde

$$
R(X, \mu)=R(x, y, \mu)=\left(r_{1}(x, y, \mu), r_{2}(x, y, \mu)\right)
$$


com

$$
\begin{aligned}
& r_{1}(x, y, \mu)=g_{1}^{-}(x, y)+\varphi^{\mu}(y)\left(g_{1}^{+}(x, y)-g_{1}^{-}(x, y)\right), \\
& r_{2}(x, y, \mu)=g_{2}^{-}(x, y)+\varphi^{\mu}(y)\left(g_{2}^{+}(x, y)-g_{2}^{-}(x, y)\right),
\end{aligned}
$$

é uma família a um parâmetro de campos de vetores suaves.

A fim de estudar os ciclos limites de (4.4) podemos usar um tipo de função de Melnikov dada no seguinte teorema.

Teorema 4.1.2. Para cada $\mu>0$, se a $>0$ é um zero simples da função

$$
\mathcal{R}(a, \mu)=\mathcal{R}^{+}(a, \mu)+\mathcal{R}^{-}(a, \mu)
$$

onde

$$
\mathcal{R}^{+}(a, \mu)=\int_{0}^{\pi}\left[r_{1}(a \cos (s), a \operatorname{sen}(s), \mu) \cos (s)+r_{2}(a \cos (s), a \operatorname{sen}(s), \mu) \operatorname{sen}(s)\right] \mathrm{d} s
$$

$e$

$$
\mathcal{R}^{-}(a, \mu)=\int_{\pi}^{2 \pi}\left[r_{1}(a \cos (s), a \operatorname{sen}(s), \mu) \cos (s)+r_{2}(a \cos (s), a \operatorname{sen}(s), \mu) \operatorname{sen}(s)\right] \mathrm{d} s,
$$

então para $\varepsilon>0$ suficientemente pequeno existe um ciclo limite $X^{\varepsilon, \mu}$ de (4.4) tal que $X^{\varepsilon, \mu}$ tende ao círculo com centro na origem e raio a quando $\varepsilon$ tende a 0 . O ciclo limite é estável se $\mathcal{R}_{a}(a, \mu)<0$ e instável se $\mathcal{R}_{a}(a, \mu)>0$.

Uma questão natural pode ser formulada sobre a relação entre as Funções de Melnikov (4.3) e (4.7). O próximo resultado fornece uma resposta nesta direção.

Teorema 4.1.3. As funções (4.3) e (4.7) dos Teoremas 4.1.1 e 4.1.2, respectivamente, satisfazem para cada $a>0$ a seguinte relação

$$
\lim _{\mu \rightarrow 0} \mathcal{R}(a, \mu)=\mathcal{M}(a)
$$

Como uma consequência da prova do Teorema (4.1.3) temos o seguinte teorema.

Teorema 4.1.4. Considere as hipóteses dos Teoremas 4.1.1 e 4.1.2. Para cada zero simples a da função (4.3) existe $\mu_{0}>0$ tal que, para toda função de regularização $\varphi$, a função (4.7) tem um zero simples $a(\mu)$, para cada $0<\mu<\mu_{0}$.

\section{Prova do Teorema 4.1.1}

A prova do Teorema 4.1.1 baseia-se principalmente no estudo do desenvolvimento de Taylor da função de separação definida pela aplicação de primeiro retorno de Poincaré, no uso do Teorema 
da Função Implícita e da diferenciabilidade e continuidade das soluções de equações diferenciais ordinárias com relação aos parâmetros e condições iniciais.

Considere a reta de separação dada por

$$
\Sigma=\mathcal{H}^{-1}(0)=\left\{(x, y) \in \mathbb{R}^{2}: y=0\right\}
$$

que pode ser escrita como $\Sigma=L^{-} \cup\{(0,0)\} \cup L^{+}$, onde

$$
L^{-}=\left\{(x, y) \in \mathbb{R}^{2}: x<0, y=0\right\}, \quad L^{+}=\left\{(x, y) \in \mathbb{R}^{2}: x>0, y=0\right\}
$$

Além disso, considere os conjuntos

$$
\Sigma^{-}=\mathcal{H}^{-1}(-\infty, 0)=\left\{(x, y) \in \mathbb{R}^{2}: y<0\right\}, \quad \Sigma^{+}=\mathcal{H}^{-1}(0,+\infty)=\left\{(x, y) \in \mathbb{R}^{2}: y>0\right\}
$$

Veja que dado $x_{0}>0$ então existe $\varepsilon>0$ suficientemente pequeno tal que para todo ponto $X \in\left\{(x, 0):-x_{0} \leq x \leq x_{0}, x \neq 0\right\} \subset L^{-} \cup L^{+}$é do tipo costura (ver Seção 2.2). De fato, tomando $N=\nabla \mathcal{H}(X)=(0,1)$ temos

$$
\left(X^{-}(X) \cdot N\right)\left(X^{+}(X) \cdot N\right)=x^{2}+\varepsilon x\left(g_{2}^{-}(x, 0)+g_{2}^{+}(x, 0)\right)+\varepsilon^{2} g_{2}^{-}(x, 0) g_{2}^{+}(x, 0)>0,
$$

para $\varepsilon>0$ suficientemente pequeno.

Observação 4.1.1. Para $\varepsilon=0$ uma solução de (4.1) com condição inicial $X_{0}$ em $\Sigma^{-}$ou $\Sigma^{+}$ intercepta $\Sigma$ transversalmente, segue da continuidade das soluções com relação aos parâmetros e condições iniciais, que para $\varepsilon>0$ suficientemente pequeno existe uma bola aberta de centro $X_{0}$ e raio $\varepsilon, B\left(X_{0}, \varepsilon\right)$ em $\Sigma^{-}$ou $\Sigma^{+}$, onde para todo ponto $Y_{0} \in B\left(X_{0}, \varepsilon\right)$ a solução de (4.1) com condição inicial $Y_{0}$ também interceptará $\Sigma$ transversalmente.

Se tomarmos a seguinte mudança de coordenadas (coordenadas polares)

$$
x+i y \mapsto r e^{i s}, \quad r \geq 0, \quad s \in[0,2 \pi],
$$

então (4.1) pode ser escrita na forma

$$
\dot{r}=\varepsilon\left\{\begin{array}{ll}
P^{+}(s, r), & s \in[0, \pi], \\
P^{-}(s, r), & s \in[\pi, 2 \pi],
\end{array} \quad \dot{s}=1+\varepsilon \begin{cases}Q^{+}(s, r), & s \in[0, \pi], \\
Q^{-}(s, r), & s \in[\pi, 2 \pi],\end{cases}\right.
$$

onde

$$
\begin{aligned}
& P^{ \pm}(s, r)=g_{1}^{ \pm}(r \cos (s), r \operatorname{sen}(s)) \cos (s)+g_{2}^{ \pm}(r \cos (s), r \operatorname{sen}(s)) \operatorname{sen}(s), \\
& Q^{ \pm}(s, r)=\frac{g_{2}^{ \pm}(r \cos (s), r \operatorname{sen}(s)) \cos (s)-g_{1}^{ \pm}(r \cos (s), r \operatorname{sen}(s)) \operatorname{sen}(s)}{r} .
\end{aligned}
$$

Para $\varepsilon_{0}>0$ suficientemente pequeno, denote por

$$
(s, a, \varepsilon) \in[0, \pi] \times L^{+} \times\left[0, \varepsilon_{0}\right] \mapsto \rho^{+}(s, a, \varepsilon)
$$


a solução de

$$
\left\{\begin{array}{l}
\frac{d}{d s} r(s)=\frac{\varepsilon P^{+}(s, r(s))}{1+\varepsilon Q^{+}(s, r(s))}, \\
r(0)=a,
\end{array}\right.
$$

e

$$
(s, b, \varepsilon) \in[\pi, 2 \pi] \times L^{-} \times\left[0, \varepsilon_{0}\right] \mapsto \rho^{-}(s, b, \varepsilon)
$$

a solução de

$$
\left\{\begin{array}{l}
\frac{d}{d s} r(s)=\frac{\varepsilon P^{-}(s, r(s))}{1+\varepsilon Q^{-}(s, r(s))}, \\
r(\pi)=b .
\end{array}\right.
$$

As (famílias a um parâmetro de) aplicações de transição

$$
\begin{aligned}
\Pi^{+}: L^{+} & \longrightarrow L^{-} \\
a & \longmapsto \Pi^{+}(a, \varepsilon)=\rho^{+}(\pi, a, \varepsilon), \\
\Pi^{-}: L^{-} & \longrightarrow L^{+} \\
b & \longmapsto \Pi^{-}(b, \varepsilon)=\rho^{-}(2 \pi, b, \varepsilon),
\end{aligned}
$$

estão bem definidas, bem como a (família a um parâmetro de) aplicação de Poincaré ou de primeiro retorno

$$
\begin{aligned}
\Pi: L^{+} & \longrightarrow L^{+} \\
a \longmapsto \Pi(a, \varepsilon) & =\left(\Pi^{-} \circ \Pi^{+}\right)(a, \varepsilon) \\
& =\rho^{-}\left(2 \pi, \rho^{+}(\pi, a, \varepsilon), \varepsilon\right) .
\end{aligned}
$$

Usando a aplicação de Poincaré acima definimos uma família de funções do tipo separação dada por

$$
\begin{aligned}
\delta: L^{+} \times\left[0, \varepsilon_{0}\right] & \longrightarrow \mathbb{R} \\
(a, \varepsilon) & \longmapsto \delta(a, \varepsilon)=\Pi(a, \varepsilon)-a .
\end{aligned}
$$

Resulta que

$$
\delta(a, 0) \equiv 0,
$$

pois

$$
\rho^{+}(\pi, a, 0)=-a, \quad \forall a \in L^{+}
$$

e

$$
\rho^{-}(2 \pi, b, 0)=-b, \quad \forall b \in L^{-} .
$$

Assim,

$$
\begin{aligned}
\delta(a, 0) & =\Pi(a)-a \\
& =\rho^{-}\left(2 \pi, \rho^{+}(\pi, a, 0), 0\right)-a \\
& =\rho^{-}(2 \pi,-a, 0)-a=-(-a)-a=0,
\end{aligned}
$$


$\forall a \in L^{+}$. A expansão de Taylor da função $\delta$ com respeito a $\varepsilon \in\left[0, \varepsilon_{0}\right]$ é dada por

$$
\delta(a, \varepsilon)=\varepsilon \delta_{\varepsilon}(a, 0)+O\left(a, \varepsilon^{2}\right)=\varepsilon\left(\delta_{\varepsilon}(a, 0)+O(a, \varepsilon)\right)=\varepsilon \Delta(a, \varepsilon),
$$

onde

$$
\delta_{\varepsilon}(a, 0)=\left.\frac{\partial}{\partial \varepsilon} \delta(a, \varepsilon)\right|_{\varepsilon=0}
$$

Segue também que

$$
\delta_{a}(a, 0) \equiv 0
$$

$\forall a \in L^{+}$, pois

$$
\left.\Pi_{a}(a, \varepsilon)\right|_{\varepsilon=0}=\left.\left(1+\varepsilon \Delta_{a}(a, \varepsilon)\right)\right|_{\varepsilon=0}=1,
$$

onde

$$
\delta_{a}(a, 0)=\left.\frac{\partial}{\partial a} \delta(a, \varepsilon)\right|_{\varepsilon=0}, \quad \Pi_{a}(a, \varepsilon)=\frac{\partial}{\partial a} \Pi(a, \varepsilon), \quad \Delta_{a}(a, \varepsilon)=\frac{\partial}{\partial a} \Delta(a, \varepsilon) .
$$

Em virtude disso, não podemos aplicar o Teorema da Função Implícita diretamente. Porém, se $a_{0}$ é zero simples da função $\Delta$ calculada em $\varepsilon=0$, isto é,

$$
\Delta\left(a_{0}, 0\right)=0 \quad\left(\delta_{\varepsilon}\left(a_{0}, 0\right)=0\right), \quad \Delta_{a}\left(a_{0}, 0\right) \neq 0 \quad\left(\delta_{a \varepsilon}\left(a_{0}, 0\right) \neq 0\right)
$$

então pelo Teorema da Função Implícita, existe um intervalo $I \subset\left[0, \varepsilon_{0}\right]$ e uma única função

$$
\begin{aligned}
\phi: I \longrightarrow L^{+} \\
\varepsilon \longmapsto a=\phi(\varepsilon)
\end{aligned}
$$

tal que

$$
\phi(0)=a_{0}, \quad \Delta(\phi(\varepsilon), \varepsilon)=0,
$$

para todo $\varepsilon \in I$, e

$$
a=\phi(\varepsilon)=a_{0}-\frac{\Delta_{\varepsilon}\left(a_{0}, 0\right)}{\Delta_{a}\left(a_{0}, 0\right)} \varepsilon+O\left(\varepsilon^{2}\right) .
$$

Como $\delta(a, \varepsilon)=\varepsilon \Delta(a, \varepsilon)$ e $a=\phi(\varepsilon)$, então

$$
\delta_{\varepsilon}(a, \varepsilon)=\Delta(a, \varepsilon)+\varepsilon\left(\Delta_{a}(a, \varepsilon) \phi^{\prime}(\varepsilon)+\Delta_{\varepsilon}(a, \varepsilon)\right) .
$$

Portanto para $\varepsilon=0$ temos

$$
\delta_{\varepsilon}\left(a_{0}, 0\right)=\Delta\left(a_{0}, 0\right)
$$

Pelos cálculos acima é suficiente estudar os zeros simples da função $\delta_{\varepsilon}(a, 0)$, pois, para todo $\varepsilon \in I$ obtemos $\Delta(\phi(\varepsilon), \varepsilon)=0$, ou seja, $\delta(\phi(\varepsilon), \varepsilon)=0$.

Como $\delta(a, \varepsilon)=\Pi(a, \varepsilon)-a=\rho^{-}\left(2 \pi, \rho^{+}(\pi, a, \varepsilon), \varepsilon\right)-a$, então

$$
\delta_{\varepsilon}(a, \varepsilon)=\rho_{b}^{-}(2 \pi, b, \varepsilon) b_{\varepsilon}+\rho_{\varepsilon}^{-}(2 \pi, b, \varepsilon)
$$


$\operatorname{com} b=\rho^{+}(\pi, a, \varepsilon)$ e $b_{\varepsilon}=\rho_{\varepsilon}^{+}(\pi, a, \varepsilon)$. Calculando em $\varepsilon=0$ obtemos

$$
\delta_{\varepsilon}(a, 0)=\rho_{b}^{-}\left(2 \pi, b^{0}, 0\right) b_{\varepsilon}^{0}+\rho_{\varepsilon}^{-}\left(2 \pi, b^{0}, 0\right)
$$

$\operatorname{com} b^{0}=\rho^{+}(\pi, a, 0)=-a$ e $b_{\varepsilon}^{0}=\rho_{\varepsilon}^{+}(\pi, a, 0)$.

Lema 4.1.1. A função $\rho_{\varepsilon}^{+}=\rho_{\varepsilon}^{+}(s, a, 0)$ é solução de

$$
\left\{\begin{array}{l}
\frac{d}{d s} \rho_{\varepsilon}^{+}(s, a, 0)=P^{+}(s, a), \\
\rho_{\varepsilon}^{+}(0, a, 0)=0,
\end{array}\right.
$$

onde $P^{+}(s, a)=P^{+}\left(s, \rho^{+}(s, a, 0)\right)$. Além disso,

$$
b_{\varepsilon}^{0}=\rho_{\varepsilon}^{+}(\pi, a, 0)=\int_{0}^{\pi} P^{+}(s, a) \mathrm{d} s .
$$

Demonstração: A função $\rho^{+}(s, a, \varepsilon)$ é solução de (4.12), isto é,

$$
\left\{\begin{array}{l}
\frac{d}{d s} \rho^{+}(s, a, \varepsilon)=\frac{\varepsilon P^{+}\left(s, \rho^{+}(s, a, \varepsilon)\right)}{1+\varepsilon Q^{+}\left(s, \rho^{+}(s, a, \varepsilon)\right)}, \\
\rho^{+}(0, a, \varepsilon)=a .
\end{array}\right.
$$

Derivando esta equação e a condição inicial com respeito ao parâmetro $\varepsilon$ e calculando em $\varepsilon=0$ obtemos a primeira parte do lema. Segue, portanto do Teorema Fundamental do Cálculo que

$$
\rho_{\varepsilon}^{+}(s, a, 0)-\rho_{\varepsilon}^{+}(0, a, 0)=\int_{t=0}^{t=s} P^{+}(t, a) \mathrm{d} t .
$$

Tomando $s=\pi$ na expressão acima a segunda parte fica demonstrada.

Lema 4.1.2. A função $\rho_{\varepsilon}^{-}=\rho_{\varepsilon}^{-}\left(s, b^{0}, 0\right)$ é solução de

$$
\left\{\begin{array}{l}
\frac{d}{d s} \rho_{\varepsilon}^{-}\left(s, b^{0}, 0\right)=P^{-}(s, a), \\
\rho_{\varepsilon}^{-}\left(\pi, b^{0}, 0\right)=0
\end{array}\right.
$$

onde $P^{-}(s, a)=P^{-}\left(s, \rho^{-}(s, a, 0)\right)$ e $b^{0}$ é como em (4.16). Além disso,

$$
\rho_{\varepsilon}^{-}\left(2 \pi, b^{0}, 0\right)=\int_{\pi}^{2 \pi} P^{-}(s, a) \mathrm{d} s .
$$

Demonstração: A função $\rho^{-}(s, b, \varepsilon)$ é solução de (4.14), isto é,

$$
\left\{\begin{array}{l}
\frac{d}{d s} \rho^{-}(s, b, \varepsilon)=\frac{\varepsilon P^{-}\left(s, \rho^{-}(s, b, \varepsilon)\right)}{1+\varepsilon Q^{-}\left(s, \rho^{-}(s, b, \varepsilon)\right)}, \\
\rho^{-}(\pi, b, \varepsilon)=b .
\end{array}\right.
$$


Derivando esta equação e a condição inicial com respeito ao parâmetro $\varepsilon$ e calculando em $\varepsilon=0$ obtemos a primeira parte do lema. Segue, portanto do Teorema Fundamental do Cálculo que

$$
\rho_{\varepsilon}^{-}\left(s, b^{0}, 0\right)-\rho_{\varepsilon}^{-}\left(\pi, b^{0}, 0\right)=\int_{t=\pi}^{t=s} P^{-}(t, a) \mathrm{d} t .
$$

Tomando $s=2 \pi$ na expressão acima a segunda parte fica demonstrada.

Lema 4.1.3. A funçãoo $\rho_{b}^{-}=\rho_{b}^{-}\left(s, b^{0}, 0\right)$ é solução de

$$
\left\{\begin{array}{l}
\dot{\rho}_{b}^{-}\left(s, b^{0}, 0\right)=0 \\
\rho_{b}^{-}\left(\pi, b^{0}, 0\right)=1
\end{array}\right.
$$

onde $b^{0}$ é como em (4.16). Além disso,

$$
\rho_{b}^{-}\left(2 \pi, b^{0}, 0\right)=1
$$

Demonstração: A função $\rho^{-}(s, b, \varepsilon)$ é solução de (4.14), isto é,

$$
\left\{\begin{array}{l}
\frac{d}{d s} \rho^{-}(s, b, \varepsilon)=\frac{\varepsilon P^{-}\left(s, \rho^{-}(s, b, \varepsilon)\right)}{1+\varepsilon Q^{-}\left(s, \rho^{-}(s, b, \varepsilon)\right)} \\
\rho^{-}(\pi, b, \varepsilon)=b .
\end{array}\right.
$$

Derivando a equação e a condição inicial com respeito a $b$ e calculando em $\varepsilon=0$ obtemos a primeira parte do lema. Assim

$$
\rho_{b}^{-}\left(s, b^{0}, 0\right)=1
$$

Tomando $s=2 \pi$ na expressão acima a segunda parte fica demonstrada.

Da equação (4.16), Lemas 4.1.1, 4.1.2 e 4.1.3 temos

$$
\begin{aligned}
\delta_{\varepsilon}(a, 0) & =\rho_{b}^{-}\left(2 \pi, b^{0}, 0\right) \rho_{\varepsilon}^{+}(\pi, a, 0)+\rho_{\varepsilon}^{-}\left(2 \pi, b^{0}, 0\right) \\
& =\int_{0}^{\pi} P^{+}(s, a) \mathrm{d} s+\int_{\pi}^{2 \pi} P^{-}(s, a) \mathrm{d} s .
\end{aligned}
$$

Usando as notações de (4.10) segue que $\mathcal{M}(a)=\delta_{\varepsilon}(a, 0)$, para $a>0$, e a relação (4.3) está provada. Agora, segue da definição da função de separação que

$$
\Pi(a, \varepsilon)=a+\varepsilon \Delta(a, \varepsilon)
$$


Diferenciando a expressão acima com respeito ao parâmetro $a$ e avaliando em $a=\phi(\varepsilon)$ obtemos

$$
\begin{aligned}
\Pi_{a}(\phi(\varepsilon), \varepsilon) & =1+\varepsilon \Delta_{a}(\phi(\varepsilon), \varepsilon) \\
& =1+\varepsilon\left(\Delta_{a}\left(a_{0}, 0\right)+O(\varepsilon)\right) \\
& =1+\varepsilon\left(\delta_{a \varepsilon}\left(a_{0}, 0\right)+O(\varepsilon)\right) \\
& =1+\mathcal{M}^{\prime}\left(a_{0}\right) \varepsilon+O\left(\varepsilon^{2}\right),
\end{aligned}
$$

após a expansão de Taylor da função $\Delta_{a}$ com respeito a $\varepsilon \in I$. Assim, o sinal de $\mathcal{M}^{\prime}\left(a_{0}\right)$ determina se $\left|\Pi_{a}(\phi(\varepsilon), \varepsilon)\right|<1$ ou $\left|\Pi_{a}(\phi(\varepsilon), \varepsilon)\right|>1$ ocorre e o Teorema 4.1 .1 está provado.

Exemplo 4.1.1. Considere o sistema

$$
\dot{X}= \begin{cases}A X+\varepsilon G^{-}(X), & y \leq 0, \\ A X+\varepsilon G^{+}(X), & y \geq 0,\end{cases}
$$

onde $X=(x, y) \in \mathbb{R}^{2}, \varepsilon \geq 0$ um parâmetro real,

$$
A=\left(\begin{array}{cc}
0 & -1 \\
1 & 0
\end{array}\right)
$$

$e$

$$
\begin{aligned}
G: & \mathbb{R}^{2} \longrightarrow \mathbb{R}^{2} \\
X & \longmapsto G(X)= \begin{cases}G^{-}(X), & y \leq 0, \\
G^{+}(X), & y \geq 0,\end{cases}
\end{aligned}
$$

onde $G^{-}(x, y)=\left(0, p(x) y-\left(k_{1} x+k_{2}\right)\right)$ e $G^{+}(x, y)=\left(0, p(x) y+\left(k_{1} x+k_{2}\right)\right)$ onde $k_{1}, k_{2} \in \mathbb{R}$, $k_{2} \neq 0$ e p é um polinômio de grau $n$, dado por

$$
p(x)=\sum_{i=0}^{n} p_{i} x^{i}
$$

Se $1-\varepsilon k_{1} \neq 0$ o equilíbrio do sistema (4.20) para $y \leq 0$ é dado por

$$
X_{0}^{-}=\left(x_{0}^{-}, 0\right),
$$

onde

$$
x_{0}^{-}=\frac{\varepsilon k_{2}}{1-\varepsilon k_{1}},
$$

e se $1+\varepsilon k_{1} \neq 0$ o equilíbrio para $y \geq 0$ é dado por

$$
X_{0}^{+}=\left(x_{0}^{+}, 0\right),
$$

onde

$$
x_{0}^{+}=-\frac{\varepsilon k_{2}}{1+\varepsilon k_{1}} .
$$


Agora, se $1-\varepsilon k_{1}=0$ o sistema (4.20) para $y \leq 0$ não tem equilíbrios pois $k_{2} \neq 0$ e para $y \geq 0$ o equilíbrio é dado por

$$
Y_{0}^{+}=\left(-\frac{\varepsilon k_{2}}{2}, 0\right)
$$

e se $1+\varepsilon k_{1}=0$ o sistema (4.20) para $y \geq 0$ não tem equilibrios pois $k_{2} \neq 0$ e para $y \leq 0$ o equilíbrio é dado por

$$
Y_{0}^{-}=\left(\frac{\varepsilon k_{2}}{2}, 0\right) .
$$

Com o intuito de aplicar o Teorema 4.1.1, precisamos observar que a origem não é equilíbrio do sistema perturbado (4.20). A aplicação deste teorema poderá ser feita desde que evitemos os pontos no conjunto de separação $\Sigma=\left\{(x, y) \in \mathbb{R}^{2}: y=0\right\}$ que não são de costura. Analisemos, primeiramente, os pontos $X_{0}^{-}, X_{0}^{+} e(0,0)$. Para o ponto $X_{0}^{-}$temos

$$
A X_{0}^{-}+\varepsilon G^{-}\left(X_{0}^{-}\right)=(0,0), \quad A X_{0}^{-}+\varepsilon G^{+}\left(X_{0}^{-}\right)=\left(0,2 x_{0}^{-}\right),
$$

$\log o$

$$
\left(\left(A X_{0}^{-}+\varepsilon G^{-}\left(X_{0}^{-}\right)\right) \cdot(0,1)\right)\left(\left(A X_{0}^{-}+\varepsilon G^{+}\left(X_{0}^{-}\right)\right) \cdot(0,1)\right)=0 .
$$

Para o ponto $X_{0}^{+}$temos

$$
A X_{0}^{+}+\varepsilon G^{-}\left(X_{0}^{+}\right)=\left(0,2 x_{0}^{+}\right), \quad A X_{0}^{+}+\varepsilon G^{+}\left(X_{0}^{+}\right)=(0,0),
$$

$\log o$

$$
\left(\left(A X_{0}^{+}+\varepsilon G^{-}\left(X_{0}^{+}\right)\right) \cdot(0,1)\right)\left(\left(A X_{0}^{+}+\varepsilon G^{+}\left(X_{0}^{+}\right)\right) \cdot(0,1)\right)=0 .
$$

Portanto $X_{0}^{-}$e $X_{0}^{+}$são pontos de tangência.

Agora, para $(0,0)$ obtemos

$$
\left(\left(A(0,0)+\varepsilon G^{-}(0,0)\right) \cdot(0,1)\right)\left(\left(A(0,0)+\varepsilon G^{+}(0,0)\right) \cdot(0,1)\right)=-\varepsilon^{2} k_{2}^{2},
$$

$\operatorname{logo}(0,0)$ é ponto de deslize.

Consideremos $X_{0}=\left(x_{0}, 0\right) \in \Sigma-\left\{X_{0}^{-}, X_{0}^{+},(0,0)\right\}$ e o intervalo $S_{k_{1}, k_{2}}^{\varepsilon}=\left(x_{0}^{\mp}, x_{0}^{ \pm}\right) . \quad S e$ $x_{0} \in S_{k_{1}, k_{2}}^{\varepsilon}$, temos que $X_{0}$ é ponto de deslize pois

$$
\left(\left(A X_{0}+\varepsilon G^{-}\left(X_{0}\right)\right) \cdot(0,1)\right)\left(\left(A X_{0}+\varepsilon G^{+}\left(X_{0}\right)\right) \cdot(0,1)\right)<0 .
$$

Assim, $X_{0}=\left(x_{0}, 0\right) \in \Sigma-\left\{X_{0}^{-}, X_{0}^{+},(0,0)\right\}$ é ponto de costura se $x_{0} \notin S_{k_{1}, k_{2}}^{\varepsilon}$.

Para o ponto $Y_{0}^{-}$temos

$$
A Y_{0}^{-}+\varepsilon G^{-}\left(Y_{0}^{-}\right)=(0,0), \quad A Y_{0}^{-}+\varepsilon G^{+}\left(Y_{0}^{-}\right)=\left(0, \varepsilon k_{2}\right),
$$

$\log o$

$$
\left(\left(A Y_{0}^{-}+\varepsilon G^{-}\left(Y_{0}^{-}\right)\right) \cdot(0,1)\right)\left(\left(A Y_{0}^{-}+\varepsilon G^{+}\left(Y_{0}^{-}\right)\right) \cdot(0,1)\right)=0 .
$$


Para o ponto $Y_{0}^{+}$temos

$$
A Y_{0}^{+}+\varepsilon G^{-}\left(Y_{0}^{+}\right)=\left(0,-\varepsilon k_{2}\right), \quad A Y_{0}^{+}+\varepsilon G^{+}\left(Y_{0}^{+}\right)=(0,0),
$$

$\log o$

$$
\left(\left(A Y_{0}^{+}+\varepsilon G^{-}\left(Y_{0}^{+}\right)\right) \cdot(0,1)\right)\left(\left(A Y_{0}^{+}+\varepsilon G^{+}\left(Y_{0}^{+}\right)\right) \cdot(0,1)\right)=0 .
$$

Portanto $Y_{0}^{-}$e $Y_{0}^{+}$são pontos de tangência.

Suponhamos que $1-\varepsilon k_{1}=0$ e considere $X_{0}=\left(x_{0}, 0\right) \in \Sigma-\left\{Y_{0}^{-}, Y_{0}^{+}\right\}$. Obtemos

$$
\left(\left(A X_{0}+\varepsilon G^{-}\left(X_{0}\right)\right) \cdot(0,1)\right)\left(\left(A X_{0}+\varepsilon G^{+}\left(X_{0}\right)\right) \cdot(0,1)\right)=\left(-\varepsilon k_{2}\right)\left(2 x_{0}+\varepsilon k_{2}\right) .
$$

Se $k_{2}<0, X_{0}$ é ponto de costura se

$$
x_{0}>-\frac{\varepsilon k_{2}}{2}>0
$$

e se $k_{2}>0, X_{0}$ é ponto de costura se

$$
x_{0}<-\frac{\varepsilon k_{2}}{2}<0
$$

Agora, se $1+\varepsilon k_{1}=0$,

$$
\left(\left(A X_{0}+\varepsilon G^{-}\left(X_{0}\right)\right) \cdot(0,1)\right)\left(\left(A X_{0}+\varepsilon G^{+}\left(X_{0}\right)\right) \cdot(0,1)\right)=\left(2 x_{0}-\varepsilon k_{2}\right)\left(\varepsilon k_{2}\right) .
$$

Se $k_{2}<0, X_{0}$ é ponto de costura se

$$
x_{0}<\frac{\varepsilon k_{2}}{2}<0
$$

e se $k_{2}>0, X_{0}$ é ponto de costura se

$$
x_{0}>\frac{\varepsilon k_{2}}{2}>0
$$

Se $1 \pm \varepsilon k_{1}=0$ novamente $(0,0)$ é ponto de deslize.

Em [32] (com algumas adaptações aqui), os autores usam a Teoria da Média (Averaging) para provar o seguinte resultado.

Proposição 4.1.1. Para todo $n \geq 1$ e $\varepsilon>0$ suficientemente pequeno o número máximo de ciclos limites de (4.20) bifurcando de órbitas periódicas do centro linear $\dot{x}=-y, \dot{y}=x$ é $[n / 2]+1$, onde [.] denota a função parte inteira. Além disso, existem sistemas (4.20) tendo exatamente $[n / 2]+1$.

Demonstração: Calculando a função $\mathcal{M}(a)$ dada no Teorema 4.1.1 obtemos 


$$
\begin{aligned}
\mathcal{M}(a) & =\int_{0}^{\pi} \sum_{i=0}^{n} p_{i}\left(a^{i+1} \cos ^{i}(s)\right) \operatorname{sen}^{2}(s) \mathrm{d} s+k_{1} a \int_{0}^{\pi} \operatorname{sen}(s) \cos (s) \mathrm{d} s+k_{2} \int_{0}^{\pi} \operatorname{sen}(s) \mathrm{d} s+ \\
& +\int_{\pi}^{2 \pi} \sum_{i=0}^{n} p_{i}\left(a^{i+1} \cos ^{i}(s)\right) \operatorname{sen}^{2}(s) \mathrm{d} s-k_{1} a \int_{\pi}^{2 \pi} \operatorname{sen}(s) \cos (s) \mathrm{d} s-k_{2} \int_{\pi}^{2 \pi} \operatorname{sen}(s) \mathrm{d} s .
\end{aligned}
$$

Portanto,

$$
\mathcal{M}(a)=\int_{0}^{2 \pi} \sum_{i=0}^{n} p_{i}\left(a^{i+1} \cos ^{i}(s)\right) \operatorname{sen}^{2}(s) \mathrm{d} s+4 k_{2} .
$$

Veja que, para todo $k=0,1,2 \ldots$

$$
\begin{gathered}
\int_{0}^{2 \pi} \cos ^{2 k+1}(s) \operatorname{sen}^{2}(s)=0, \\
\int_{0}^{2 \pi} \cos ^{2 k}(s) \operatorname{sen}^{2}(s)=\pi \alpha_{k}, \alpha_{k} \in \mathbb{R} .
\end{gathered}
$$

Logo,

$$
\mathcal{M}(a)=\sum_{\substack{i=0, i \text { par }}}^{n} \pi p_{i} \alpha_{i} a^{i+1}+4 k_{2}
$$

Para simplificar definimos

$$
\alpha_{i}:=\frac{\pi \alpha_{i}}{k_{2}}
$$

assim,

$$
\mathcal{M}(a)=0 \Leftrightarrow-\sum_{\substack{i=0, i \text { par }}}^{n} p_{i} \alpha_{i} a^{i+1}=4 .
$$

Se $n$ é par temos

$$
-a\left(p_{0} \alpha_{0}+p_{2} \alpha_{2} a^{2}+p_{4} \alpha_{4} a^{4}+\ldots+p_{n} \alpha_{n} a^{n}\right)=4,
$$

e se $n$ é ímpar

$$
-a\left(p_{0} \alpha_{0}+p_{2} \alpha_{2} a^{2}+p_{4} \alpha_{4} a^{4}+\ldots+p_{n-1} \alpha_{n-1} a^{n-1}\right)=4
$$

Os polinômios no $1^{\circ}$ membro de (4.27) e (4.28) têm grau ímpar, além disso, ambos têm zero com raiz e portanto, estes polinômios têm o mesmo número de raízes. Vamos considerar o caso em que $n$ é par (o caso $n$ ímpar é igualmente tratado) e provar a existência de no máximo $(n / 2)+1$ ciclos limites bifurcando das órbitas periódicas do centro linear (se $n$ é ímpar temos 
$[n / 2]+1$ ciclos), isto é, para $n \geq 1$ e $\varepsilon>0$ suficientemente pequeno este é o número máximo de órbitas periódicas que persistem após a perturbação do centro linear.

Denotemos

$$
P(a)=-a P_{0}(a)
$$

onde $P_{0}(a)=p_{0} \alpha_{0}+p_{2} \alpha_{2} a^{2}+p_{4} \alpha_{4} a^{4}+\ldots+p_{n} \alpha_{n} a^{n}$ e $n$ é par.

Veja que:

(a) $P(0)=0$ e $P$ tem no máximo $n / 2$ raízes positivas, pois raízes não nulas são simétricas já que $P_{0}(a)$ só tem grau par, isto é, $P_{0}(a)$ é a soma de monômios de grau par, assim, se $a \neq 0, P(a)=0$ se, e somente se, $P_{0}(a)=0$ e $P(-a)=P(a)$ pois, $P_{0}(-a)=P_{0}(a)$.

(b) $P^{\prime}(a)$ também é dado pela soma de monômios de grau $n$ (par), ou seja, as raízes de $P^{\prime}(a)$ também são simétricas, logo existem no máximo $n / 2$ pontos críticos positivos (máximos ou minímos) para $P$.

(c) Se $a_{1}$ e $a_{2}$ são duas raízes de $P(a)=4$, pelo Teorema de Rolle, existe $a^{*} \in\left(a_{1}, a_{2}\right)$ tal que $P^{\prime}\left(a^{*}\right)=0$.

(d) Do item (b) segue que não existem mais do que duas soluções de $P(a)=4$ entre dois zeros de $P$ pois, o número de raízes e pontos críticos é no máximo $n / 2$. Dessa forma para cada raiz de $P$ temos um ponto crítico.

(e) Se $P^{\prime}(0)>0$ temos no máximo $(n / 2)+1$ soluções para $P(a)=4$. Veja Figura 4.1. De fato, seja $a_{1}>0$ a primeira raiz positiva de $P$, isto é $P\left(a_{1}\right)=0$, como $P(0)=0$, do item (d) podemos ter no intervalo $\left[0, a_{1}\right]$ duas interseções do gráfico de $P$ com a reta $Q(a)=4$. Como o número de raízes positivas de $P$ é par, temos mais uma raiz $a_{2}$ de $P$, resultando em mais uma interseção após $a_{2}$. Se essas forem as únicas raízes de $P$ teremos portanto no máximo 3 interseções. Assim se tivermos $n / 2=2 k$ raízes positivas, digamos $a_{1}, a_{2}, \ldots ., a_{2 k-2}, a_{2 k-1}$ para $a_{0}=0$ e $k=1,2,3, \ldots$ temos $k$ intervalos

$$
\left[0, a_{1}\right],\left[a_{2}, a_{3}\right], \ldots,\left[a_{2 k-2}, a_{2 k-1}\right]
$$

onde poderemos ter duas interseções em cada intervalo e uma após $a_{2 k-1}$ senão, teríamos um número ímpar de raízes positivas de $P$. Logo, podemos ter $2 k+1$ interseções, ou seja $n / 2+1$ interseções do gráfico de $P$ com a reta $Q=4$.

(f) Se $P^{\prime}(0)<0$ temos no máximo $n / 2$ soluções para $P(a)=4$. Veja Figura 4.2. De fato, seja $a_{1}>0$ a primeira raiz positiva de $P$, isto é $P\left(a_{1}\right)=0$, como $P(0)=0$, no intervalo 


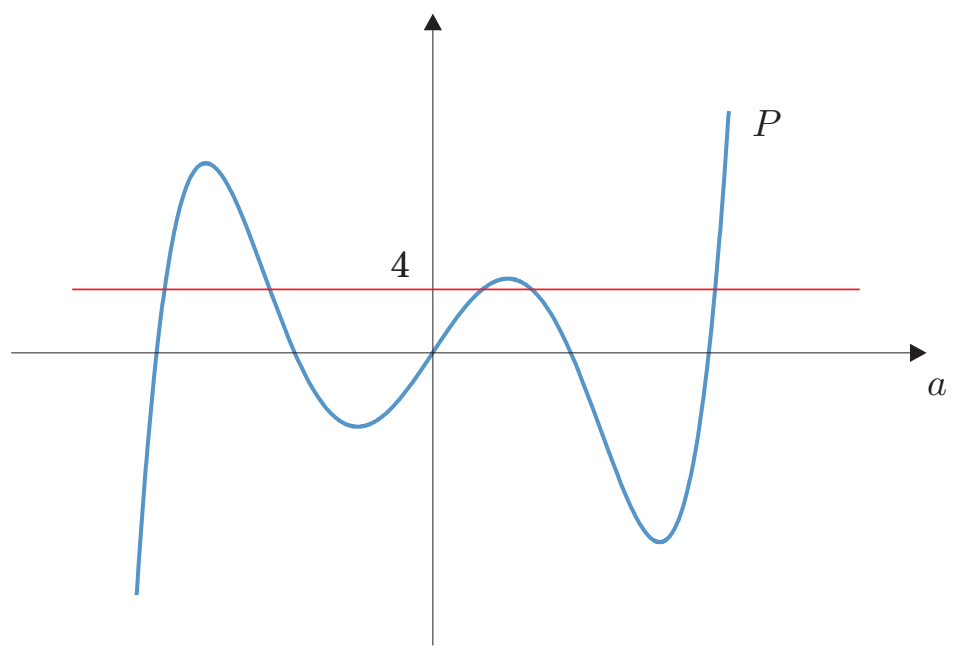

Figura 4.1: $P^{\prime}(0)>0$ e $n=4$.

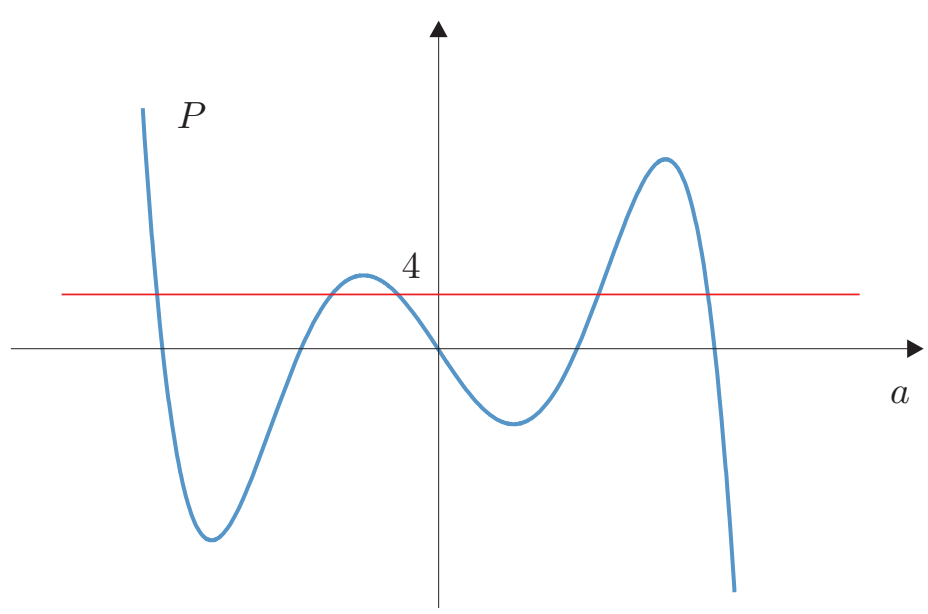

Figura 4.2: $P^{\prime}(0)<0$ e $n=4$.

$\left[0, a_{1}\right]$ não ocorre interseção do gráfico de $P$ com a reta $Q(a)=4$. Como o número de raízes positivas de $P$ é par, devemos ter mais uma raiz $a_{2}$ de $P$, do item (d) podemos ter duas interseções no intervalo $\left[a_{1}, a_{2}\right]$. Se essas forem as únicas raízes de $P$ teremos portanto no máximo 2 interseções. Assim se tivermos $n / 2=2 k$ raízes positivas, digamos $a_{1}, a_{2}, \ldots, a_{2 k-1}, a_{2 k}$ para $k=1,2,3, \ldots$ temos $k$ intervalos

$$
\left[a_{1}, a_{2}\right],\left[a_{3}, a_{4}\right], \ldots,\left[a_{2 k-1}, a_{2 k}\right]
$$

onde poderemos ter duas interseções em cada intervalo. Logo, podemos ter $2 k$ interseções, ou seja $n / 2$ interseções do gráfico de $P$ com a reta $Q=4$.

Mostremos agora que para cada $n$ existe um sistema com exatamente $(n / 2)+1$ ciclos limite. Para isto é suficiente mostrar a existência de um polinômio na forma (4.29) que satisfaz $P(a)=4$ 
para $(n / 2)+1$ valores de $a$. Fixemos $n$ par e consideremos o polinômio

$$
P(a)=\lambda a \prod_{r=1}^{n / 2}(a-r)(a+r)
$$

onde $\lambda \neq 0$.

As raízes positivas deste polinômio fornecem condições iniciais $X_{0}=(a, 0)$ para o sistema perturbado (4.20) tal que para $\varepsilon>0$ suficientemente pequeno a solução com esta condição inicial seja uma órbita periódica (ciclo limite). Como $a=1$ é a menor raiz positiva deste polinômio, sejam $x_{0}^{-}$e $x_{0}^{+}$dados em (4.22) e (4.23) respectivamente, se $x_{0}^{ \pm}>x_{0}^{\mp}$, escolha $k_{1}$ e $k_{2}$ tal que $x_{0}^{ \pm}<1$ e a solução do sistema (4.20) para $\varepsilon>0$ suficientemente pequeno com condição inicial $X_{0}=(1,0)$ dada por $X\left(t, X_{0}, \varepsilon\right)=\left(x\left(t, X_{0}, \varepsilon\right), y\left(t, X_{0}, \varepsilon\right)\right)$, intercepte $\Sigma-\{(0,0)\}$ após um tempo $t^{+}>0$, isto é, $X\left(t^{+}, X_{0}, \varepsilon\right)=\left(x\left(t^{+}, X_{0}, \varepsilon\right), 0\right)$, com $x\left(t^{+}, X_{0}, \varepsilon\right)<x_{0}^{\mp}$. Agora os casos onde $1 \pm \varepsilon k_{1}=0$ não podem acontecer, pois, pelo Teorema 4.1.1 para $\varepsilon>0$ suficientemente pequeno existe um ciclo limite $X^{\varepsilon}$ de (4.20) tal que $X^{\varepsilon}$ tende ao círculo de centro na origem e raio igual a $a$ quando $\varepsilon$ tende a 0 . Com estas escolhas garantimos que os pontos sobre o conjunto de separação são pontos de costura.

Escolha $\lambda$ tal que $P(1 / 2)>4$ e $P(1 / 20)<3$. Temos

$$
P\left(\frac{1}{2}\right)=\lambda h_{1}>4,
$$

onde

$$
h_{1}=\frac{1}{2} \prod_{r=1}^{n / 2}\left(\frac{1}{4}-r^{2}\right)
$$

e

$$
P\left(\frac{1}{20}\right)=\lambda h_{2}<3
$$

onde

$$
h_{2}=\frac{1}{20} \prod_{r=1}^{n / 2}\left(\frac{1}{400}-r^{2}\right) .
$$

(a) Se $n=4 k, k=1,2,3, \ldots$, então, $h_{1}>0$ e $h_{2}>0$ pois

$$
\begin{aligned}
& \frac{1}{4}-r^{2}<0 \\
& \frac{1}{400}-r^{2}<0
\end{aligned}
$$

e

$$
\begin{gathered}
h_{1}=\frac{1}{2} \prod_{r=1}^{2 k}\left(\frac{1}{4}-r^{2}\right), \\
h_{2}=\frac{1}{20} \prod_{r=1}^{2 k}\left(\frac{1}{400}-r^{2}\right),
\end{gathered}
$$


são portanto dados pelo produto de um número par de fatores negativos. Logo,

$$
0<\frac{4}{h_{1}}<\lambda<\frac{3}{h_{2}}
$$

Assim, se $n=4 k, \lambda>0$.

(b) Se $n=4 k-2, k=1,2,3, \ldots$, então, $h_{1}<0$ e $h_{2}<0$ pois

$$
\begin{aligned}
& \frac{1}{4}-r^{2}<0 \\
& \frac{1}{400}-r^{2}<0
\end{aligned}
$$

e

$$
\begin{gathered}
h_{1}=\frac{1}{2} \prod_{r=1}^{2 k-1}\left(\frac{1}{4}-r^{2}\right), \\
h_{2}=\frac{1}{20} \prod_{r=1}^{2 k-1}\left(\frac{1}{400}-r^{2}\right)
\end{gathered}
$$

são portanto dados pelo produto de um número ímpar de fatores negativos. Logo,

$$
\frac{3}{h_{2}}<\lambda<\frac{4}{h_{1}}<0
$$

Assim, se $n=4 k-2, \lambda<0$.

Portanto,

se $n=4 k$, escolha $\lambda>0$ no intervalo

$$
\left(\frac{4}{h_{1}}, \frac{3}{h_{2}}\right)
$$

Se $n=4 k-2$, escolha $\lambda<0$ no intervalo

$$
\left(\frac{3}{h_{2}}, \frac{4}{h_{1}}\right)
$$

Escolhendo $\lambda$ em cada caso obtemos

$$
P\left(\frac{1}{2}\right)>4 \quad \text { e } P\left(\frac{1}{20}\right)<3 .
$$

Além disso, temos que

$$
P^{\prime}(0)=\lambda \prod_{r=1}^{n / 2}\left(-r^{2}\right)
$$

Se $n=4 k, \lambda>0$ e $\prod_{r=1}^{2 k}\left(-r^{2}\right)>0, \log \mathrm{O} P^{\prime}(0)>0$.

Se $n=4 k-2, \lambda<0$ e $\prod_{r=1}^{2 k-1}\left(-r^{2}\right)<0$, logo também temos $P^{\prime}(0)>0$. 
Pela nossa construção, o gráfico de $P(a)$ intercepta a reta $Q(a)=4$ em um ponto entre 0 e $1 / 2$ e outro entre $1 / 2$ e 1 , este último por que $P(1)=0$. Logo no intervalo $[0,1]$ temos duas interseções.

Com estas hipóteses mostremos agora que o gráfico de $P(a)$ intercepta o gráfico de $Q(a)=4$ em exatamente $(n / 2)+1$ pontos. Mostremos que

$$
P\left(2 k+\frac{1}{2}\right)>4, \quad \forall k=1,2,3, \ldots
$$

De fato,

$$
4 k+1>1, \text { então, } \frac{(4 k+1)^{2}}{4}-r^{2}>\frac{1}{4}-r^{2} .
$$

Logo,

$$
P\left(2 k+\frac{1}{2}\right)=P\left(\frac{4 k+1}{2}\right)=\frac{\lambda}{2}(4 k+1) \prod_{r=1}^{n / 2}\left[\frac{(4 k+1)^{2}}{4}-r^{2}\right]>\frac{\lambda}{2} \prod_{r=1}^{n / 2}\left(\frac{1}{4}-r^{2}\right)=P\left(\frac{1}{2}\right)>4 .
$$

Se $n=4 k$, os intervalos são da forma

$$
[0,1],[2,3],[4,5], \ldots,[2 k-2,2 k-1]
$$

com $k=1,2,3, \ldots$ Em termos de $n$ temos os intervalos

$$
[0,1],[2,3],[4,5], \ldots,\left[\frac{n}{2}-2, \frac{n}{2}-1\right]
$$

$\operatorname{com} n=4,8,12, \ldots$

No intervalo $[0,1]$ ocorrem duas interseções como mostramos acima. Como $2 k+1 / 2$ é o ponto médio dos intervalos cujos extremos são raízes positivas de $P$ e $P(2 k+1 / 2)>4$, temos portanto, duas interseções em cada um dos intervalos restantes. Além disso ocorre mais uma interseção após $2 k-1$. De fato, como $n=4 k$, temos que $n / 2=2 k$, isto é, um número par de raízes positivas de $P$, porém, temos um número de $2 k-1$ raízes positivas listadas acima, ou seja um número ímpar de raízes. Portanto deve ocorrer uma outra interseção após $2 k-1$. Como temos $k$ intervalos, obtemos $2 k+1$ interseções, ou seja $n / 2+1$ interseções.

Se $n=4 k-2$, os intervalos são da forma

$$
[0,1],[2,3],[4,5], \ldots,[2 k-2,2 k-1]
$$

com $k=1,2,3, \ldots$ Em termos de $n$ temos os intervalos

$$
[0,1],[2,3],[4,5], \ldots,\left[\frac{n}{2}-1, \frac{n}{2}\right]
$$

$\operatorname{com} n=2,6,8, \ldots$

Como no caso anterior ocorrem duas interseções em cada intervalo. Além disso não ocorre interseção após $2 k-1$. De fato, como $n=4 k-2$, temos que $n / 2=2 k-1$, isto é, um número 
ímpar de raízes positivas de $P$, com temos um número de $2 k-1$ raízes positivas listadas acima, ou seja um número ímpar de raízes, não deve ocorrer uma outra interseção após $2 k-1$. Como temos $k$ intervalos, obtemos $2 k$ interseções, ou seja $n / 2+1$ interseções.

\section{Prova do Teorema 4.1.2}

Não podemos aplicar diretamente ao sistema (4.1) o Teorema 2.1.3 dado na Seção 2.1, pois o campo vetorial $G$ em (4.2) não é suave. Assim, a fim de utilizar o Teorema 2.1.3, usamos o método de regularização, introduzido na Seção 2.2. para transformar o campo de vetores $Z$ dado em (4.1) numa família a dois parâmetros de campos de vetores suaves dada por

$$
Z^{\mu}(X, \varepsilon)=A X+\varepsilon R(X, \mu)
$$

onde

$$
R(X, \mu)=R(x, y, \mu)=\left(r_{1}(x, y, \mu), r_{2}(x, y, \mu)\right)
$$

com

$$
\begin{aligned}
& r_{1}(x, y, \mu)=g_{1}^{-}(x, y)+\varphi^{\mu}(y)\left(g_{1}^{+}(x, y)-g_{1}^{-}(x, y)\right), \\
& r_{2}(x, y, \mu)=g_{2}^{-}(x, y)+\varphi^{\mu}(y)\left(g_{2}^{+}(x, y)-g_{2}^{-}(x, y)\right),
\end{aligned}
$$

é uma família a um parâmetro de campos de vetores suaves. Para $\varepsilon=0$ temos um centro linear, ou seja, o problema regularizado é uma perturbação de um centro linear. Assim, a partir do Teorema (2.1.3), para cada $\mu>0$ podemos escrever a função de Melnikov como

$$
\mathcal{R}(a, \mu)=\int_{0}^{2 \pi}\left[r_{1}(a \cos (s), a \operatorname{sen}(s), \mu) \cos (s)+r_{2}(a \cos (s), a \operatorname{sen}(s), \mu) \operatorname{sen}(s)\right] \mathrm{d} s .
$$

A função $\mathcal{R}$ acima pode ser escrita simplesmente como a soma das duas integrais $\mathcal{R}^{-}$e $\mathcal{R}^{+}$ dadas no Teorema 4.1.2, O Teorema 4.1.2 está provado.

Observação 4.1.2. A demonstração do Teorema 4.1.2 segue também do Teorema 2.2.1 quando $F(X)=(-y, x)$.

\section{Prova dos Teoremas 4.1 .3 e 4.1 .4}

Começamos apresentando sem provas dois teoremas clássicos da Análise. O primeiro teorema é uma versão do Teorema da Convergência Limitada sua prova pode ser encontrada em [3]. Ver também [34. 
Teorema 4.1.5. (Teorema da Convergência Limitada) Seja $\left\{f_{n}\right\}$ é uma sequência de funções em um intervalo $[a, b]$. Se $f_{n}, f:[a, b] \rightarrow \mathbb{R}$ são funções Riemann integráveis (para cada $n \in \mathbb{N}$ ) tal que

(a) $\lim _{n \rightarrow+\infty} f_{n}(x)=f(x)$ para todo $x$ exceto possivelmente para um subconjunto de medida nula de Lebesgue;

(b) $\sup _{x \in[a, b]}\left|f_{n}(x)\right| \leq M$ para uma constante positiva apropriada $M$.

Então,

$$
\lim _{n \rightarrow+\infty} \int_{a}^{b} f_{n}(x) \mathrm{d} x=\int_{a}^{b} f(x) d x
$$

A prova do segundo teorema pode ser encontrada em [35], página 84.

Teorema 4.1.6. Considere $W$ e $V$ espaços métricos, $E \subset W, f: W \rightarrow V$ e $p$ um ponto limite de E. Então

$$
\lim _{x \rightarrow p} f(x)=q \Longleftrightarrow \lim _{n \rightarrow+\infty} f\left(p_{n}\right)=q,
$$

para toda sequência $\left\{p_{n}\right\}$ em $E$ tal que

$$
p_{n} \neq p, \quad \lim _{n \rightarrow+\infty} p_{n}=p
$$

Começamos a prova do Teorema 4.1.3 analisando a função $\mathcal{R}$ dada em (4.7).

Se $0<s<\pi$ então $\operatorname{sen}(s)>0$. Pela continuidade da função de regularização $\varphi$ temos que

$$
\lim _{\mu \rightarrow 0} \varphi^{\mu}(a \operatorname{sen}(s))=\lim _{\mu \rightarrow 0} \varphi\left(\frac{a \operatorname{sen}(s)}{\mu}\right)=\varphi\left(\lim _{\mu \rightarrow 0} \frac{a \operatorname{sen}(s)}{\mu}\right)=1 .
$$

Assim,

$$
\lim _{\mu \rightarrow 0} r_{1}(a \cos (s), a \operatorname{sen}(s), \mu)=g_{1}^{+}(a \cos (s), a \operatorname{sen}(s))
$$

e

$$
\lim _{\mu \rightarrow 0} r_{2}(a \cos (s), a \operatorname{sen}(s), \mu)=g_{2}^{+}(a \cos (s), a \operatorname{sen}(s)) .
$$

Agora, se $\pi<s<2 \pi$ então $\operatorname{sen}(s)<0$. Pela continuidade da função de regularização $\varphi$ temos que

$$
\lim _{\mu \rightarrow 0} \varphi^{\mu}(a \operatorname{sen}(s))=\lim _{\mu \rightarrow 0} \varphi\left(\frac{a \operatorname{sen}(s)}{\mu}\right)=\varphi\left(\lim _{\mu \rightarrow 0} \frac{a \operatorname{sen}(s)}{\mu}\right)=0 .
$$

Assim,

$$
\lim _{\mu \rightarrow 0} r_{1}(a \cos (s), a \operatorname{sen}(s), \mu)=g_{1}^{-}(a \cos (s), a \operatorname{sen}(s))
$$

e

$$
\lim _{\mu \rightarrow 0} r_{2}(a \cos (s), a \operatorname{sen}(s), \mu)=g_{2}^{-}(a \cos (s), a \operatorname{sen}(s)) .
$$


Para $s=0, s=\pi$ ou $s=2 \pi$ temos $\operatorname{sen}(s)=0$. Assim, para $s=0, s=\pi$ ou $s=2 \pi$ obtemos

$$
\begin{aligned}
& \lim _{\mu \rightarrow 0} r_{1}(a \cos (s), a \operatorname{sen}(s), \mu)=g_{1}^{-}(a, 0)+\varphi(0)\left(g_{1}^{+}(a, 0)-g_{1}^{-}(a, 0)\right), \\
& \lim _{\mu \rightarrow 0} r_{2}(a \cos (s), a \operatorname{sen}(s), \mu)=g_{2}^{-}(a, 0)+\varphi(0)\left(g_{2}^{+}(a, 0)-g_{2}^{-}(a, 0)\right) .
\end{aligned}
$$

Para cada $a>0$ e $\mu>0$ fixados, defina a função $F^{+}:[0, \pi] \rightarrow \mathbb{R}$ por

$$
F^{+}(a, s, \mu)=r_{1}(a \cos (s), a \operatorname{sen}(s), \mu) \cos (s)+r_{2}(a \cos (s), a \operatorname{sen}(s), \mu) \operatorname{sen}(s) .
$$

Como $r_{1}, r_{2}, \cos (s)$ e $\operatorname{sen}(s)$ são funções contínuas em $[0, \pi]$ para todo $a>0$ e $\mu>0$ fixados, então, $F^{+}$é contínua neste intervalo. Considere a sequência

$$
F_{n}^{+}(a, s)=F^{+}\left(a, s, \mu_{n}\right)
$$

onde $\left(\mu_{n}\right)_{n \in \mathbb{N}}$ é uma sequência de números reais positivos que tende a 0 quando $n$ tende para o infinito. Para cada $n \in \mathbb{N}$ a sequência $\left\{F_{n}^{+}\right\}$é Riemann integrável em $[0, \pi]$. Temos que $F_{n}^{+}(a, s) \rightarrow F_{0}^{+}(a, s)$ quase sempre em $[0, \pi]$, onde

$$
F_{0}^{+}(a, s)=g_{1}^{+}(a \cos (s), a \operatorname{sen}(s)) \cos (s)+g_{2}^{+}(a \cos (s), a \operatorname{sen}(s)) \operatorname{sen}(s) .
$$

Além disso, $F_{0}^{+}(a, s)$ é Riemann integrável em $[0, \pi]$. A sequência $\left\{F_{n}^{+}\right\}$é limitada, pois suas funções componentes são funções contínuas em $[0, \pi]$ e definidas no compacto $K=\gamma_{a}^{+}([0, \pi])$, onde $\gamma_{a}^{+}(s)=(a \cos (s), a \sin (s))$.

Pela análise acima, estamos nas hipóteses do Teorema 4.1.5. Assim,

$$
\lim _{n \rightarrow+\infty} \int_{0}^{\pi} F_{n}^{+}(a, s) \mathrm{d} s=\int_{0}^{\pi} F_{0}^{+}(a, s) \mathrm{d} s
$$

Da mesma forma defina a função $F^{-}:[\pi, 2 \pi] \rightarrow \mathbb{R}$ por

$$
F^{-}(a, s, \mu)=r_{1}(a \cos (s), a \operatorname{sen}(s), \mu) \cos (s)+r_{2}(a \cos (s), a \operatorname{sen}(s), \mu) \operatorname{sen}(s)
$$

e a sequência

$$
F_{n}^{-}(a, s)=F^{-}\left(a, s, \mu_{n}\right)
$$

onde $\left(\mu_{n}\right)_{n \in \mathbb{N}}$ é uma sequência de números reais positivos que tende a 0 quando $n$ tende para o infinito. Com os mesmos argumentos acima, $F_{n}^{-}(a, s) \rightarrow F_{0}^{-}(a, s)$ quase sempre em $[\pi, 2 \pi]$ quando $n \rightarrow \infty$ onde

$$
F_{0}^{-}(a, s)=g_{1}^{-}(a \cos (s), a \operatorname{sen}(s)) \cos (s)+g_{2}^{-}(a \cos (s), a \operatorname{sen}(s)) \operatorname{sen}(s) .
$$

Assim, usando novamente o Teorema 4.1.5 obtemos

$$
\lim _{n \rightarrow+\infty} \int_{\pi}^{2 \pi} F_{n}^{-}(a, s) \mathrm{d} s=\int_{\pi}^{2 \pi} F_{0}^{-}(a, s) \mathrm{d} s .
$$


Portanto, usando o Teorema 4.1.6, (4.30) e (4.31) obtemos

$$
\begin{aligned}
\lim _{\mu \rightarrow 0} \mathcal{R}(a, \mu) & =\lim _{n \rightarrow+\infty} \mathcal{R}\left(a, \mu_{n}\right) \\
& =\lim _{n \rightarrow+\infty} \int_{0}^{\pi} F_{n}^{+}(a, s) \mathrm{d} s+\lim _{n \rightarrow+\infty} \int_{\pi}^{2 \pi} F_{n}^{-}(a, s) \mathrm{d} s \\
& =\int_{0}^{\pi} F_{0}^{+}(a, s) \mathrm{d} s+\int_{\pi}^{2 \pi} F_{0}^{-}(a, s) \mathrm{d} s \\
& =\mathcal{M}^{+}(a)+\mathcal{M}^{-}(a)=\mathcal{M}(a) .
\end{aligned}
$$

Em resumo o Teorema 4.1.3 está provado. A prova do teorema 4.1 .4 segue do 4.1 .3 e da condição de transversalidade para um zero simples da função de Melnikov $\mathcal{M}$.

Exemplo 4.1.2. Considere o sistema (4.1) com um campo de vetores descontínuo $G$ em (4.2) definido por

$$
G^{-}(x, y)=\left(0,\left(1-x^{2}\right) y\right), \quad G^{+}(x, y)=(x, 0) .
$$

Do Teorema 4.1.1 obtemos

$$
\mathcal{M}(a)=\int_{0}^{\pi} a \cos ^{2}(s) \mathrm{d} s+\int_{\pi}^{2 \pi} a\left(1-a^{2} \cos ^{2}(s)\right) \operatorname{sen}^{2}(s)(s) \mathrm{d} s=a \pi\left(1-\frac{a^{2}}{8}\right) .
$$

Existe um único zero positivo de $\mathcal{M}$ dado por $a=2 \sqrt{2}$. Além disso, este zero é um zero simples pois

$$
\mathcal{M}^{\prime}(2 \sqrt{2})=-2 \pi
$$

Portanto para $\varepsilon$ suficientemente pequeno existe um ciclo limite estável $X^{\varepsilon}$ de (4.1) tal que $X^{\varepsilon}$ tende ao círculo com centro na origem e raio $2 \sqrt{2}$ quando $\varepsilon$ tende a 0 . Veja a Figura 4.3.

O método de regularização produz o campo de vetores (4.4) onde

$$
R(X, \mu)=R(x, y, \mu)=\left(r_{1}(x, y, \mu), r_{2}(x, y, \mu)\right)
$$

com

$$
r_{1}(x, y, \mu)=\varphi^{\mu}(y) x, \quad r_{2}(x, y, \mu)=\left(1-\varphi^{\mu}(y)\right)\left(1-x^{2}\right) y
$$

e a função de regularização de classe $C^{1}$ dada por

$$
\varphi^{\mu}(y)=\left\{\begin{array}{l}
0, \quad y<-\mu \\
\frac{3}{4 \mu} y-\frac{y^{3}}{4 \mu^{3}}+\frac{1}{2}, \quad-\mu \leq y \leq \mu, \\
1, \quad y>\mu .
\end{array}\right.
$$

De acordo com o Teorema 4.1.2 podemos estudar os zeros simples da função

$$
\mathcal{R}(a, \mu)=\int_{0}^{2 \pi}\left[r_{1}(a \cos (s), a \operatorname{sen}(s), \mu) \cos (s)+r_{2}(a \cos (s), a \operatorname{sen}(s), \mu) \operatorname{sen}(s)\right] \mathrm{d} s .
$$




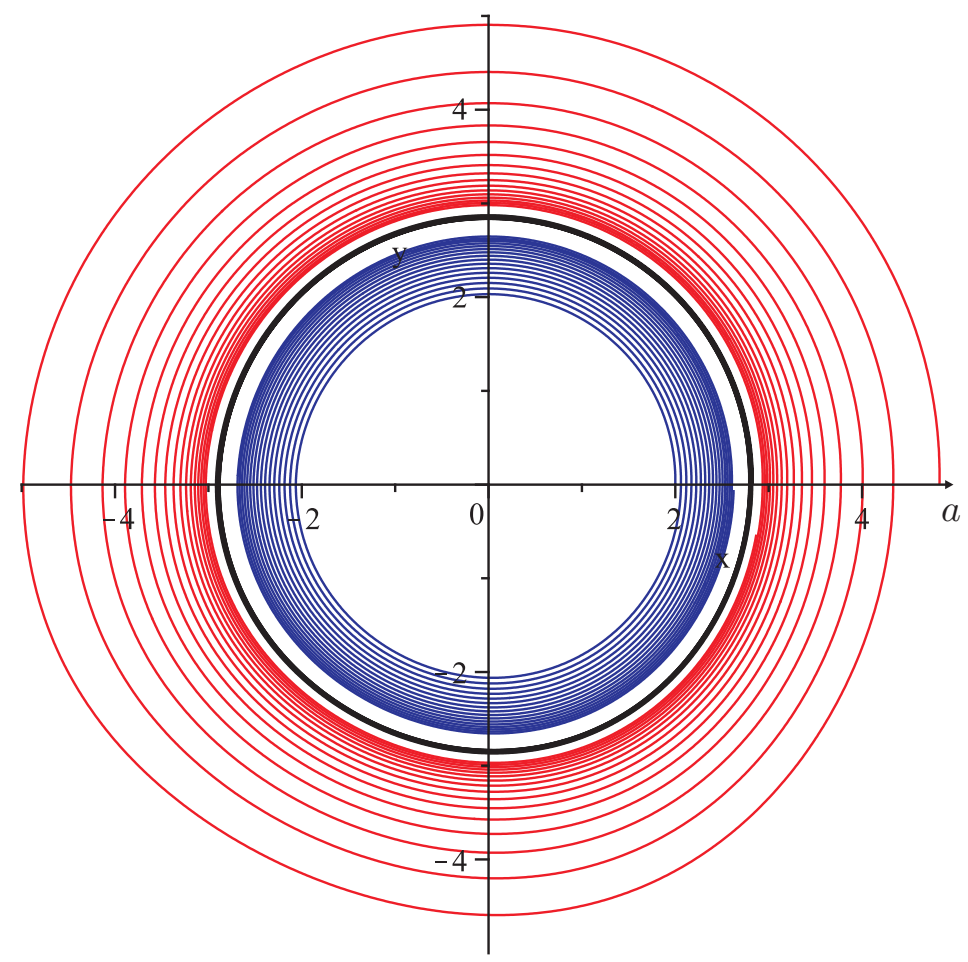

Figura 4.3: Ciclo limite (preto) do sistema perturbado para $\varepsilon=0,02$.

Da integral acima e definindo

$$
F(a, s, \mu)=r_{1}(a \cos (s), a \operatorname{sen}(s), \mu) \cos (s)+r_{2}(a \cos (s), a \operatorname{sen}(s), \mu) \operatorname{sen}(s),
$$

obtemos

$$
F(a, s, \mu)=\left[1-a^{2} \cos ^{2}(s)\right] a \operatorname{sen}^{2}(s)
$$

se $\operatorname{asen}(s)<-\mu$,

$$
F(a, s, \mu)=\varphi^{\mu}(a \operatorname{sen}(s)) a \cos ^{2}(s)+\left[1-\varphi^{\mu}(a \operatorname{sen}(s))\right]\left[1-a^{2} \cos ^{2}(s)\right] a \operatorname{sen}^{2}(s),
$$

$s e-\mu \leq a \operatorname{sen}(s) \leq \mu e$

$$
F(a, s, \mu)=a \cos ^{2}(s),
$$

se $\operatorname{asen}(s)>\mu$.

Considere a sequência $\left(\mu_{n}\right)_{n \in \mathbb{N}}$ com $\mu_{n}=1 / n$ e tome

$$
F_{n}(a, s)=F\left(a, s, \frac{1}{n}\right) \text {. }
$$

Estamos interessados no cálculo de

$$
\begin{aligned}
\mathcal{R}\left(a, \frac{1}{n}\right) & =\int_{0}^{2 \pi} F\left(a, s, \frac{1}{n}\right) \mathrm{d} s=\int_{0}^{2 \pi} F_{n}(a, s) \mathrm{d} s \\
& =\int_{0}^{\pi} F_{n}(a, s) \mathrm{d} s+\int_{\pi}^{2 \pi} F_{n}(a, s) \mathrm{d} s \\
& =\int_{0}^{\pi} F_{n}^{+}(a, s) \mathrm{d} s+\int_{\pi}^{2 \pi} F_{n}^{-}(a, s) \mathrm{d} s=\mathcal{R}^{+}\left(a, \frac{1}{n}\right)+\mathcal{R}^{-}\left(a, \frac{1}{n}\right) .
\end{aligned}
$$


Com as notações acima obtemos

$$
F_{n}(a, s)=\left[1-a^{2} \cos ^{2}(s)\right] a \operatorname{sen}^{2}(s)
$$

se $\operatorname{asen}(s)<-1 / n$,

$$
F_{n}(a, s)=\varphi^{1 / n}(a \operatorname{sen}(s)) a \cos ^{2}(s)+\left[1-\varphi^{1 / n}(a \operatorname{sen}(s))\right]\left[1-a^{2} \cos ^{2}(s)\right] a \operatorname{sen}^{2}(s),
$$

$s e-1 / n \leq a \operatorname{sen}(s) \leq 1 / n e$

$$
F_{n}(a, s)=a \cos ^{2}(s)
$$

se $\operatorname{asen}(s)>1 / n$, onde

$$
\varphi^{1 / n}(a \operatorname{sen}(s))=\left\{\begin{array}{l}
0, \quad a \operatorname{sen}(s)<-\frac{1}{n} \\
\frac{3}{4\left(\frac{1}{n}\right)} a \operatorname{sen}(s)-\frac{(a \operatorname{sen}(s))^{3}}{4\left(\frac{1}{n}\right)^{3}}+\frac{1}{2}, \quad-\frac{1}{n} \leq a \operatorname{sen}(s) \leq \frac{1}{n}, \\
1, \quad a \operatorname{sen}(s)>\frac{1}{n} .
\end{array}\right.
$$

Se $s \in[0, \pi]$ então asen $(s) \geq 0$. Para este caso, com a notação $F_{n}^{+}(a, s)=F_{n}(a, s)$, obtemos

$$
F_{n}^{+}(a, s)=\varphi^{1 / n}(a \operatorname{sen}(s)) a \cos ^{2}(s)+\left[1-\varphi^{1 / n}(a \operatorname{sen}(s))\right]\left[1-a^{2} \cos ^{2}(s)\right] a \operatorname{sen}^{2}(s),
$$

se $0 \leq a \operatorname{sen}(s) \leq 1 / n e$

$$
F_{n}^{+}(a, s)=a \cos ^{2}(s)
$$

se $\operatorname{asen}(s)>1 / n$.

Agora, se $s \in[\pi, 2 \pi]$ então asen $(s) \leq 0$. Para este caso, com a notação $F_{n}^{-}(a, s)=F_{n}(a, s)$, obtemos

$$
F_{n}^{-}(a, s)=\left[1-a^{2} \cos ^{2}(s)\right] a \operatorname{sen}^{2}(s)
$$

se $\operatorname{asen}(s)<-1 / n e$

$$
F_{n}^{-}(a, s)=\varphi^{1 / n}(a \operatorname{sen}(s)) a \cos ^{2}(s)+\left[1-\varphi^{1 / n}(a \operatorname{sen}(s))\right]\left[1-a^{2} \cos ^{2}(s)\right] a \operatorname{sen}^{2}(s)(s)
$$

se $-1 / n \leq a \operatorname{sen}(s) \leq 0$.

Resolvendo a equação asen $(s)=1 / n$ para $s \in(0, \pi)$ obtemos

$$
s_{1}=\operatorname{arcsen}\left(\frac{1}{a n}\right), \quad s_{2}=\pi-\operatorname{arcsen}\left(\frac{1}{a n}\right) .
$$

Veja Figura 4.4.

Podemos calcular

$$
\mathcal{R}^{+}\left(a, \frac{1}{n}\right)=\int_{0}^{\pi} F_{n}^{+}(a, s) \mathrm{d} s
$$


pela soma de três integrais

$$
\begin{gathered}
\int_{0}^{s_{1}}\left[\varphi^{1 / n}(a \operatorname{sen}(s)) a \cos ^{2}(s)+\left[1-\varphi^{1 / n}(a \operatorname{sen}(s))\right]\left[1-a^{2} \cos ^{2}(s)\right] a \operatorname{sen}^{2}(s)\right] \mathrm{d} s \\
\int_{s_{1}}^{s_{2}} a \cos ^{2}(s) \mathrm{d} s
\end{gathered}
$$

$e$

$$
\int_{s_{2}}^{\pi}\left[\varphi^{1 / n}(a \operatorname{sen}(s)) a \cos ^{2}(s)+\left[1-\varphi^{1 / n}(a \operatorname{sen}(s))\right]\left[1-a^{2} \cos ^{2}(s)\right] a \operatorname{sen}^{2}(s)\right] \mathrm{d} s .
$$

Após alguns cálculos, obtemos

$$
\lim _{n \rightarrow+\infty} \mathcal{R}^{+}\left(a, \frac{1}{n}\right)=\lim _{n \rightarrow+\infty} \int_{0}^{\pi} F_{n}^{+}(a, s) \mathrm{d} s=\frac{\pi a}{2} .
$$

Resolvendo a equação asen $(s)=-1 / n$ para $s \in(\pi, 2 \pi)$ obtemos

$$
s_{3}=\pi+\operatorname{arcsen}\left(\frac{1}{a n}\right), \quad s_{4}=2 \pi-\operatorname{arcsen}\left(\frac{1}{a n}\right) .
$$

Veja Figura 4.4.

Podemos calcular novamente

$$
\mathcal{R}^{-}\left(a, \frac{1}{n}\right)=\int_{\pi}^{2 \pi} F_{n}^{-}(a, s) \mathrm{d} s
$$

pela soma das três integrais seguintes

$$
\begin{gathered}
\int_{\pi}^{s_{3}}\left[\varphi^{1 / n}(a \operatorname{sen}(s)) a \cos ^{2}(s)+\left[1-\varphi^{1 / n}(a \operatorname{sen}(s))\right]\left[1-a^{2} \cos ^{2}(s)\right] a \operatorname{sen}^{2}(s)\right] \mathrm{d} s \\
\int_{s_{3}}^{s_{4}}\left[1-a^{2} \cos ^{2}(s)\right] a \operatorname{sen}^{2}(s) \mathrm{d} s
\end{gathered}
$$

$e$

$$
\int_{s_{4}}^{2 \pi}\left[\varphi^{1 / n}(a \operatorname{sen}(s)) a \cos ^{2}(s)+\left[1-\varphi^{1 / n}(a \operatorname{sen}(s))\right]\left[1-a^{2} \cos ^{2}(s)\right] a \operatorname{sen}^{2}(s)\right] \mathrm{d} s .
$$

Após alguns cálculos, obtemos

$$
\lim _{n \rightarrow+\infty} \mathcal{R}^{-}\left(a, \frac{1}{n}\right)=\lim _{n \rightarrow+\infty} \int_{\pi}^{2 \pi} F_{n}^{-}(a, s) \mathrm{d} s=\frac{\pi a}{2}-\frac{\pi}{8} a^{3} .
$$

Portanto, usando os resultados acima obtemos

$$
\begin{aligned}
\lim _{n \rightarrow+\infty} \mathcal{R}\left(a, \frac{1}{n}\right) & =\lim _{n \rightarrow+\infty} \mathcal{R}^{+}\left(a, \frac{1}{n}\right)+\lim _{n \rightarrow+\infty} \mathcal{R}^{-}\left(a, \frac{1}{n}\right) \\
& =\frac{\pi a}{2}+\left(\frac{\pi a}{2}-\frac{\pi}{8} a^{3}\right)=a \pi\left(1-\frac{a^{2}}{8}\right) \\
& =\mathcal{M}(a) .
\end{aligned}
$$




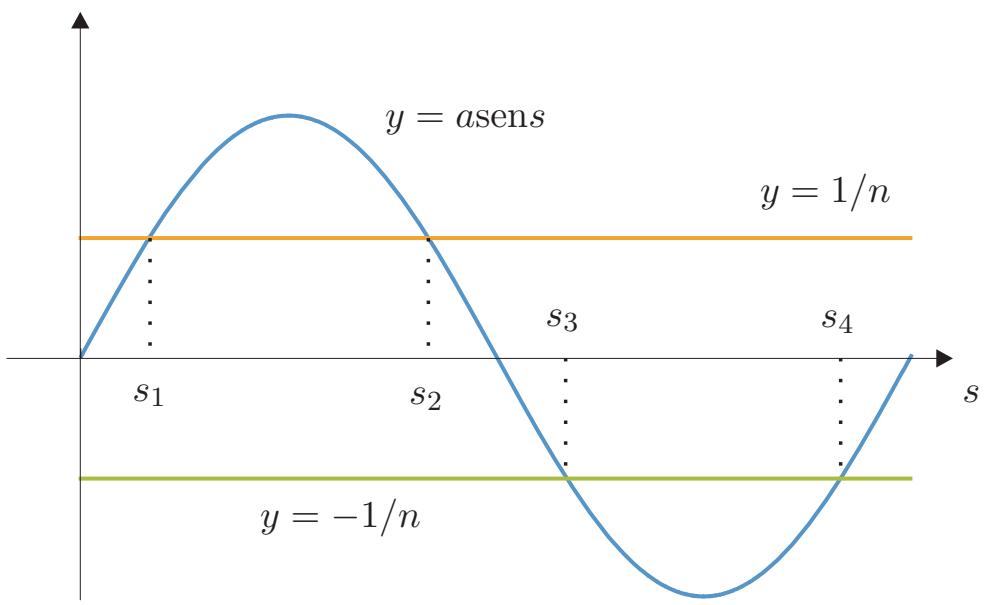

Figura 4.4: Soluções da equação $a \operatorname{sen}(s)= \pm 1 / n$.

\subsection{Centro Hamiltoniano Descontínuo Perturbado por Partes}

Considere o sistema

$$
\dot{X}=\frac{d X}{d t}=Z(X, \varepsilon)=F(X)+\varepsilon G(X)
$$

com $X=(x, y) \in \mathbb{R}^{2}, \varepsilon \geq 0$ um parâmetro real suficientemente pequeno, o ponto denotando a derivada com respeito ao parâmetro $t$, chamada aqui de tempo,

$$
\begin{aligned}
F: & \mathbb{R}^{2} \longrightarrow \mathbb{R}^{2} \\
X & \longmapsto F(X)= \begin{cases}F^{-}(X), & y \leq 0, \\
F^{+}(X), & y \geq 0,\end{cases}
\end{aligned}
$$

e

$$
\begin{aligned}
G: & \mathbb{R}^{2} \longrightarrow \mathbb{R}^{2} \\
X & \longmapsto G(X)= \begin{cases}G^{-}(X), & y \leq 0, \\
G^{+}(X), & y \geq 0,\end{cases}
\end{aligned}
$$

sendo

$$
F^{-}(x, y)=\left(-H_{y}^{-}(x, y), H_{x}^{-}(x, y)\right), \quad F^{+}(x, y)=\left(-H_{y}^{+}(x, y), H_{x}^{+}(x, y)\right)
$$

e

$$
G^{-}(x, y)=\left(g_{1}^{-}(x, y), g_{2}^{-}(x, y)\right), \quad G^{+}(x, y)=\left(g_{1}^{+}(x, y), g_{2}^{+}(x, y)\right)
$$

campos vetoriais suaves (classe $C^{k}, k \geq 1$ ), $H^{ \pm}: U \rightarrow \mathbb{R}, U$ aberto de $\mathbb{R}^{2}$, suave (classe $C^{k}$, $k \geq 1$ ). O sistema (4.32) é uma perturbação por partes de um sistema Hamiltoniano suave por partes que chamaremos, simplesmente, de sistema Hamiltoniano descontínuo perturbado por partes. Suponhamos que (4.32) para $\varepsilon=0$ temos um centro Hamiltoniano suave por partes em torno da origem e consideremos as seguintes hipóteses: 
(I) Existe um intervalo $I=(0, \alpha)$ e dois pontos diferentes da origem

$$
P(a)=(p(a), 0) \quad \text { e } \quad Q(a)=(q(a), 0)
$$

tal que para todo $a \in I$

$$
H^{+}(P(a))=H^{+}(Q(a))=a \quad \text { e } \quad H^{-}(P(a))=H^{-}(Q(a))
$$

onde $P(a) \neq Q(a)$ com $P$ e $Q$ suaves.

(II) Para $y \geq 0$ temos um arco de solução $\Gamma_{a}^{+}$com origem em $P(a)$ e extremidade em $Q(a)$, parametrizado pela função $\gamma_{a}^{+}$e definido por

$$
H^{+}(x, y)=a,
$$

e para $y \leq 0$ tem um arco de solução $\Gamma_{a}^{-}$com origem em $Q(a)$ e extremidade em $P(a)$, parametrizado pela função $\gamma_{a}^{-}$e definido por

$$
H^{-}(x, y)=H^{-}(Q(a))
$$

(III) Os arcos de solução $\Gamma_{a}^{+}$e $\Gamma_{a}^{-}$interceptam o eixo- $x$ somente nos pontos $P(a)$ e $Q(a)$ para todo $a \in I$. Além disso, sem perda de generalidade, suponhamos que estes arcos de solução tenham orientação anti-horária.

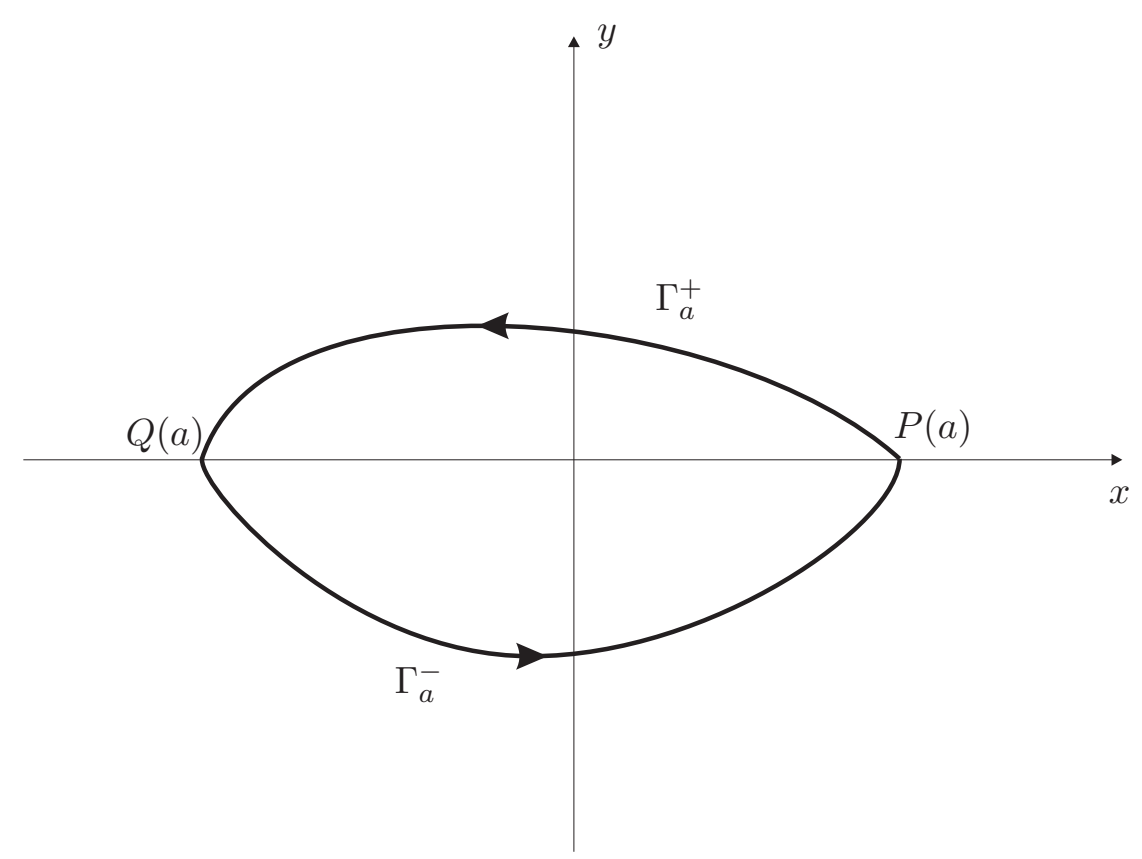

Figura 4.5: Órbitas periódicas do sistema não perturbado. 
Com as hipóteses (I), (II) e (III) o sistema (4.32) para $\varepsilon=0$ tem uma família a um parâmetro de órbitas periódicas

$$
\Gamma_{a}=\Gamma_{a}^{+} \cup \Gamma_{a}^{-}, \quad \text { para } a \in I,
$$

onde cada curva fechada $\Gamma_{a}$ é suave por partes em torno da origem com orientação anti-horária. Suponhamos que $\Gamma_{a}$ seja parametrizada pela família de funções $\gamma_{a}$. Veja a Figura 4.5. Pela construção acima os pontos sobre o conjunto de separação exceto a origem são pontos de costura. Então, para $\varepsilon>0$ suficientemente pequeno as órbitas do sistema perturbado (4.32) têm a mesma propriedade. Veja Observação 4.1.1.

Considere as órbitas do sistema (4.32) iniciando em $P(a)$. Sejam

$$
Q^{\varepsilon}(a)=\left(q^{\varepsilon}(a), 0\right) \quad \text { e } \quad P^{\varepsilon}(a)=\left(p^{\varepsilon}(a), 0\right)
$$

pontos das órbitas que interceptam o eixo $x<0$ e $x>0$ respectivamente, onde $P^{0}(a)=P(a)$ e $Q^{0}(a)=Q(a)$.

Agora, defina a função $\delta(a, \varepsilon)$ do sistema perturbado (4.32) por

$$
H^{+}\left(P^{\varepsilon}(a)\right)-H^{+}(P(a))=\delta(a, \varepsilon) .
$$

Veja que as funções $H^{+}, P^{\varepsilon}$ e $Q^{\varepsilon}$ (para $\varepsilon>0$ suficientemente pequeno) são suaves em $(a, \varepsilon)$, então a função $\delta(a, \varepsilon)$ também é suave.

A expansão de Taylor da função $\delta$ com respeito a $\varepsilon$ em torno de $\varepsilon=0$ é dada por

$$
\delta(a, \varepsilon)=\varepsilon \delta_{\varepsilon}(a, 0)+O\left(a, \varepsilon^{2}\right)=\varepsilon\left(\delta_{\varepsilon}(a, 0)+O(a, \varepsilon)\right)=\varepsilon \Delta(a, \varepsilon)
$$

sendo

$$
\delta_{\varepsilon}(a, 0)=\left.\frac{\partial}{\partial \varepsilon} \delta(a, \varepsilon)\right|_{\varepsilon=0} .
$$

Assim,

$$
H^{+}\left(P^{\varepsilon}(a)\right)-H^{+}(P(a))=\varepsilon \Delta(a, \varepsilon) .
$$

Veja que a função $P(a) \rightarrow P^{\varepsilon}(a)$ é a aplicação de Poincaré do sistema perturbado (4.32). Veja a Figura 4.6 .

Segue de (4.37), das hipóteses (I), (II) e (III) e do Teorema da Função Implícita que um zero (um zero simples, respectivamente) da função $\Delta$ corresponde a uma órbita periódica (um ciclo limite, respectivamente) do sistema perturbado (4.32). Temos, portanto o seguinte lema.

Lema 4.2.1. Para $\varepsilon>0$ suficientemente pequeno e $a \in I, \Delta \in C^{k}, k \geq 1$. Além disso, o sistema perturbado (4.32) tem uma órbita periódica (um ciclo limite, respectivamente) $\Gamma_{a_{0}}$ para $a_{0} \in I$ se, e somente se, $\Delta$ tem um zero (um zero simples, respectivamente) para a próximo de $a_{0}$. 


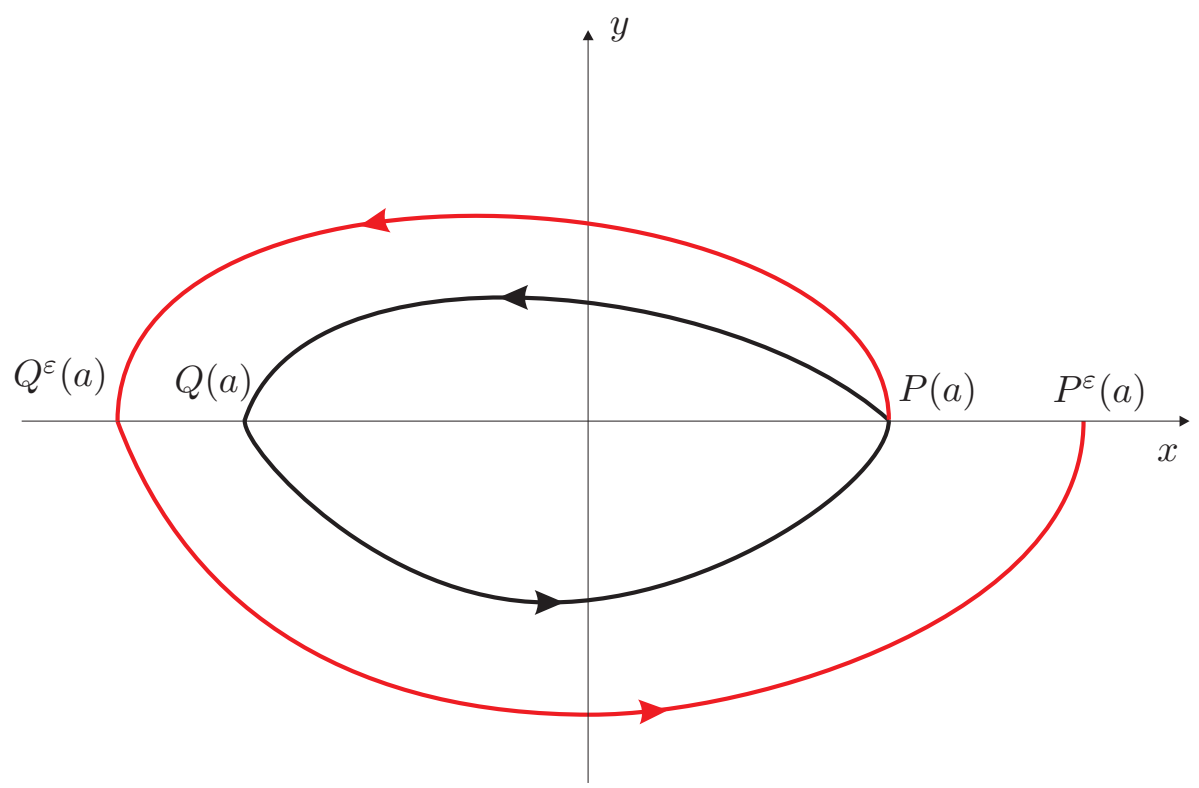

Figura 4.6: Aplicação de Poincaré.

Seja $\mathcal{M}(a)=\Delta(a, 0)=\delta_{\varepsilon}(a, 0)$. Como no caso suave, chamamos $\mathcal{M}(a)$ de função de Melnikov de primeira ordem do sistema perturbado (4.32).

Teorema 4.2.1. Sob as hipóteses (I), (II) e (III), a função de Melnikov de primeira ordem para o sistema (4.32) é dada por

$$
\mathcal{M}(a)=\frac{H_{x}^{+}(P(a))}{H_{x}^{-}(P(a))}\left[\frac{H_{x}^{-}(Q(a))}{H_{x}^{+}(Q(a))} \oint_{\gamma_{a}^{+}} g_{1}^{+} \mathrm{d} y-g_{2}^{+} \mathrm{d} x+\oint_{\gamma_{a}^{-}} g_{1}^{-} \mathrm{d} y-g_{2}^{-} \mathrm{d} x\right] .
$$

Além disso, se $a_{0} \in I$ é um zero simples positivo da função $\mathcal{M}$, então para $\varepsilon>0$ suficientemente pequeno existe um ciclo limite $X^{\varepsilon}$ tal que $X^{\varepsilon}$ tende a órbita periódica $\Gamma_{a_{0}}$ quando $\varepsilon$ tende a 0 . O ciclo limite é estável se $\mathcal{M}^{\prime}\left(a_{0}\right)<0$ e instável se $\mathcal{M}^{\prime}\left(a_{0}\right)>0$.

O Teorema 4.2.1 pode ser encontrado em uma forma similar em [24], onde os autores consideram o conjunto de separação dado pelo eixo-y.

Se no sistema (4.32) tivermos $g_{1}^{ \pm}(x, y)=g_{1}(x, y)$ e $g_{2}^{ \pm}(x, y)=g_{2}(x, y)$, do Teorema 4.2.1 segue que a função de Melnikov é neste caso

$$
\mathcal{M}(a)=\frac{H_{x}^{+}(P(a))}{H_{x}^{-}(P(a))}\left[\frac{H_{x}^{-}(Q(a))}{H_{x}^{+}(Q(a))} \oint_{\gamma_{a}^{+}} g_{1} \mathrm{~d} y-g_{2} \mathrm{~d} x+\oint_{\gamma_{a}^{-}} g_{1} \mathrm{~d} y-g_{2} \mathrm{~d} x\right] .
$$

Agora, se no sistema (4.32) temos $H^{+}(x, y)=H^{-}(x, y)$, segue imediatamente do Teorema 4.2.1 que a função de Melnikov é dada por

$$
\mathcal{M}(a)=\oint_{\gamma_{a}^{+}} g_{1}^{+} \mathrm{d} y-g_{2}^{+} \mathrm{d} x+\oint_{\gamma_{a}^{-}} g_{1}^{-} \mathrm{d} y-g_{2}^{-} \mathrm{d} x .
$$

Observação 4.2.1. Da função de Melnikov (4.40) e das conclusões do Teorema 4.2.1 obtemos imediatamente o Teorema 2.1 .3 da seção anterior parametrizando os arcos $\Gamma_{a}^{+}$e $\Gamma_{a}^{-}$em (4.35) como arcos de circunferências de raios iguais a a. 
Suponhamos que no sistema (4.32) tenhamos $H^{+}(x, y)=H^{-}(x, y)=H(x, y)$, ou seja, juntamente com as hipóteses (I), (II) e (III) temos um centro Hamiltoniano quando $\varepsilon=0$. Portanto, existe uma família de órbitas periódicas $\Gamma_{a}$ do sistema (4.32) quando $\varepsilon=0$ definida por

$$
\Gamma_{a}: H(x, y)=a, a \in I
$$

parametrizadas por $\gamma_{a}(s)$, com $s \in[0, T(a, 0)]$ onde $T(a, 0)>0$ é a função período. Logo, para $s=0$, temos $\gamma_{a}(0)=P(a)$. Suponhamos também que $\gamma_{a}^{+}(s), s \in\left[0, T^{+}(a, 0)\right]$ e $\gamma_{a}^{-}(s)$, $s \in\left[T^{+}(a, 0), T^{-}(a, 0)\right]$ são parametrizações dos arcos de trajetórias definidos para $y \geq 0 \mathrm{e}$ $y \leq 0$ respectivamente, onde $T^{+}(a, 0)>0$ e $T^{-}(a, 0)>0$ são as funções semi-período tal que $\gamma_{a}^{+}(0)=\gamma_{a}^{-}\left(T^{-}(a, 0)\right)=P(a)$ e $\gamma_{a}^{-}\left(T^{+}(a, 0)\right)=\gamma_{a}^{+}\left(T^{+}(a, 0)\right)=Q(a)$.

Estamos interessados novamente em aplicar o método de regularização, a fim de obter uma função de Melnikov do sistema regularizado e obter os mesmos resultados da seção anterior.

Aplicando o método de regularização neste caso obtemos o campo regularizado dado por

$$
Z^{\mu}(X, \varepsilon)=F(X)+\varepsilon R(X, \mu)
$$

onde

$$
F(X)=\left(-H_{y}(x, y), H_{x}(x, y)\right),
$$

com

$$
R(X, \mu)=R(x, y, \mu)=\left(r_{1}(x, y, \mu), r_{2}(x, y, \mu)\right)
$$

onde

$$
\begin{aligned}
& r_{1}(x, y, \mu)=g_{1}^{-}(x, y)+\varphi^{\mu}(y)\left(g_{1}^{+}(x, y)-g_{1}^{-}(x, y)\right), \\
& r_{2}(x, y, \mu)=g_{2}^{-}(x, y)+\varphi^{\mu}(y)\left(g_{2}^{+}(x, y)-g_{2}^{-}(x, y)\right) .
\end{aligned}
$$

Como $\operatorname{div} F=0$, segue da equação (2.47) do Teorema 2.2.1 que a função de Melnikov do sistema que define o campo regularizado $Z^{\mu}$ é

$$
\mathcal{R}(a, \mu)=\oint_{\gamma_{a}} r_{1}(x, y, \mu) \mathrm{d} y-r_{2}(x, y, \mu) \mathrm{d} x
$$

onde $\gamma_{a}(s)=\left(x_{a}(s), y_{a}(s)\right)$ e $s \in[0, T(a, 0)]$.

Os Teoremas 4.1.1 e 4.1.2 da seção anterior se repetem aqui trocando as equações $\mathcal{M}$ e $\mathcal{R}$ nestes Teoremas por (4.40) e (4.42) nas construções dadas acima. O enunciado do Teorema 4.1.4 é exatamente o mesmo e sua prova depende da prova do Teorema 4.1.3, que enunciamos novamente a seguir.

Teorema 4.2.2. As funções (4.40) e (4.42), nas construções acima, satisfazem para cada $a \in I$ a seguinte relação

$$
\lim _{\mu \rightarrow 0} \mathcal{R}(a, \mu)=\mathcal{M}(a) .
$$


Considere agora a regularização do campo $Z$ definido pelo sistema (4.32). Obtemos o campo regularizado

$$
Z^{\mu}(X, \varepsilon)=F(X, \mu)+\varepsilon R(X, \mu)
$$

onde

$$
F(X, \mu)=\left(f_{1}(X, \mu), f_{2}(X, \mu)\right)=\left(-H_{y}^{-}+\varphi^{\mu}(y)\left(H_{y}^{-}-H_{y}^{+}\right), H_{x}^{-}+\varphi^{\mu}(y)\left(H_{x}^{+}-H_{x}^{-}\right)\right)
$$

com

$$
R(X, \mu)=R(x, y, \mu)=\left(r_{1}(x, y, \mu), r_{2}(x, y, \mu)\right)
$$

onde

$$
\begin{aligned}
& r_{1}(x, y, \mu)=g_{1}^{-}(x, y)+\varphi^{\mu}(y)\left(g_{1}^{+}(x, y)-g_{1}^{-}(x, y)\right), \\
& r_{2}(x, y, \mu)=g_{2}^{-}(x, y)+\varphi^{\mu}(y)\left(g_{2}^{+}(x, y)-g_{2}^{-}(x, y)\right) .
\end{aligned}
$$

O teorema a seguir da condições para que o campo $Z^{\mu}$ em (4.44) para $\varepsilon=0$ seja um campo Hamiltoniano.

Teorema 4.2.3. Para cada $\mu>0$ e $\varepsilon=0$ o campo (4.44) é Hamiltoniano se $H_{x}^{+}(x, y)=$ $H_{x}^{-}(x, y)$. Além disso, sob estas hipóteses, é sempre possível obter uma função Hamiltoniana $\widetilde{H}(x, y, \mu)$ para o sistema regularizado.

\section{Prova do Teorema 4.2.1}

Veja que

$$
\begin{aligned}
H^{+}\left(P^{\varepsilon}\right)-H^{+}(P(a))= & {\left[H^{+}\left(P^{\varepsilon}\right)-H^{-}\left(P^{\varepsilon}\right)\right]+\left[H^{-}\left(P^{\varepsilon}\right)-H^{-}\left(Q^{\varepsilon}\right)\right] } \\
+ & {\left[H^{-}\left(Q^{\varepsilon}\right)-H^{+}\left(Q^{\varepsilon}\right)\right]+\left[H^{+}\left(Q^{\varepsilon}\right)-H^{+}(P(a))\right] } \\
\equiv & l_{1}+l_{2}+l_{3}+l_{4} . \\
l_{4}=\left[H^{+}\left(Q^{\varepsilon}\right)-H^{+}(P(a))\right]= & \int_{\widehat{P Q^{\varepsilon}}} \mathrm{d} H^{+}=\int_{\widehat{P Q^{\varepsilon}}} H_{x}^{+} \mathrm{d} x+H_{y}^{+} \mathrm{d} y \\
= & \int_{\widehat{P Q^{\varepsilon}}}\left[H_{x}^{+} \frac{\mathrm{d} x}{\mathrm{~d} t}+H_{y}^{+} \frac{\mathrm{d} y}{\mathrm{~d} t}\right] \mathrm{d} t \\
= & \int_{\widehat{P Q^{\varepsilon}}}\left[H_{x}^{+}\left(-H_{y}^{+}+\varepsilon g_{1}^{+}\right)+H_{y}^{+}\left(H_{x}^{+}+\varepsilon g_{2}^{+}\right)\right] \mathrm{d} t \\
& =\varepsilon \int_{\widehat{P Q^{\varepsilon}}}^{\varepsilon} g_{1}^{+} \mathrm{d} y-g_{2}^{+} \mathrm{d} x .
\end{aligned}
$$

Tomando a expansão de Taylor da função $l_{4}$ com respeito a $\varepsilon$, em torno de $\varepsilon=0$ obtemos

$$
\left.\frac{\partial l_{4}}{\partial \varepsilon}\right|_{\varepsilon=0}=\int_{\widehat{P Q}} g_{1}^{+} \mathrm{d} y-g_{2}^{+} \mathrm{d} x
$$


Por outro lado,

$$
\frac{\partial l_{4}}{\partial \varepsilon}=\frac{\partial}{\partial \varepsilon}\left[H^{+}\left(Q^{\varepsilon}\right)-H^{+}(P(a))\right]=\frac{\partial}{\partial \varepsilon} H^{+}\left(Q^{\varepsilon}\right)=H_{x}^{+}(q(a, \varepsilon), 0) \frac{\partial}{\partial \varepsilon} q(a, \varepsilon) .
$$

Então,

$$
\left.\frac{\partial l_{4}}{\partial \varepsilon}\right|_{\varepsilon=0}=\left.H_{x}^{+}(Q(a)) \frac{\partial}{\partial \varepsilon} q(a, \varepsilon)\right|_{\varepsilon=0} .
$$

Como as órbitas interceptam $\Sigma$ transversalmente, devemos ter $H_{x}^{+}(Q(a)) \neq 0$. De (4.45) e (4.46) segue que

$$
\left.\frac{\partial}{\partial \varepsilon} q(a, \varepsilon)\right|_{\varepsilon=0}=\frac{\int_{\widehat{P Q}} g_{1}^{+} \mathrm{d} y-g_{2}^{+} \mathrm{d} x}{H_{x}^{+}(Q(a))} .
$$

Similarmente, temos

$$
\begin{aligned}
l_{2}=\left[H^{-}\left(P^{\varepsilon}\right)-H^{-}\left(Q^{\varepsilon}\right)\right] & =\int_{\widehat{Q^{\varepsilon} P^{\varepsilon}}} \mathrm{d} H^{-}=\int_{\widehat{Q^{\varepsilon} P^{\varepsilon}}} H_{x}^{-} \mathrm{d} x+H_{y}^{-} \mathrm{d} y \\
& =\int_{\widehat{Q^{\varepsilon} P^{\varepsilon}}}\left[H_{x}^{-} \frac{\mathrm{d} x}{\mathrm{~d} t}+H_{y}^{-} \frac{\mathrm{d} y}{\mathrm{~d} t}\right] \mathrm{d} t \\
& =\int_{\widehat{Q^{\varepsilon} P^{\varepsilon}}}\left[H_{x}^{-}\left(-H_{y}^{-}+\varepsilon g_{1}^{-}\right)+H_{y}^{-}\left(H_{x}^{-}+\varepsilon g_{2}^{-}\right)\right] \mathrm{d} t \\
& =\varepsilon \int_{\widehat{Q^{\varepsilon} P^{\varepsilon}}} g_{1}^{-} \mathrm{d} y-g_{2}^{-} \mathrm{d} x .
\end{aligned}
$$

Expandindo em $\varepsilon$, em torno de $\varepsilon=0$ obtemos

$$
\left.\frac{\partial l_{2}}{\partial \varepsilon}\right|_{\varepsilon=0}=\int_{\widehat{Q P}} g_{1}^{-} \mathrm{d} y-g_{2}^{-} \mathrm{d} x
$$

Por outro lado,

$$
\frac{\partial l_{2}}{\partial \varepsilon}=\frac{\partial}{\partial \varepsilon}\left[H^{-}\left(P^{\varepsilon}\right)-H^{-}\left(Q^{\varepsilon}\right)\right]=H_{x}^{-}(p(a, \varepsilon), 0) \frac{\partial}{\partial \varepsilon} p(a, \varepsilon)-H_{x}^{-}(q(a, \varepsilon), 0) \frac{\partial}{\partial \varepsilon} q(a, \varepsilon) .
$$

Então,

$$
\left.\frac{\partial l_{2}}{\partial \varepsilon}\right|_{\varepsilon=0}=\left.H_{x}^{-}(P(a)) \frac{\partial}{\partial \varepsilon} p(a, \varepsilon)\right|_{\varepsilon=0}+\left.H_{x}^{-}(Q(a)) \frac{\partial}{\partial \varepsilon} q(a, \varepsilon)\right|_{\varepsilon=0} .
$$

De (4.47) e (4.49) segue que

$$
\left.\frac{\partial}{\partial \varepsilon} p(a, \varepsilon)\right|_{\varepsilon=0}=\frac{1}{H_{x}^{-}(P(a))} \int_{\widehat{Q P}} g_{1}^{-} \mathrm{d} y-g_{2}^{-} \mathrm{d} x+\frac{H_{x}^{-}(Q(a))}{H_{x}^{+}(Q(a)) H_{x}^{-}(P(a))} \int_{\widehat{P Q}} g_{1}^{+} \mathrm{d} y-g_{2}^{+} \mathrm{d} x .
$$

Também

$$
\frac{\partial l_{3}}{\partial \varepsilon}=\frac{\partial}{\partial \varepsilon}\left[H^{-}\left(Q^{\varepsilon}\right)-H^{+}\left(Q^{\varepsilon}\right)\right]=H_{x}^{-}(q(a, \varepsilon), 0) \frac{\partial}{\partial \varepsilon} q(a, \varepsilon)-H_{x}^{+}(q(a, \varepsilon), 0) \frac{\partial}{\partial \varepsilon} q(a, \varepsilon) .
$$

Então,

$$
\left.\frac{\partial l_{3}}{\partial \varepsilon}\right|_{\varepsilon=0}=\left.\left[H_{x}^{-}(Q(a))-H_{x}^{+}(Q(a))\right] \frac{\partial}{\partial \varepsilon} q(a, \varepsilon)\right|_{\varepsilon=0} .
$$

De (4.47) segue que

$$
\left.\frac{\partial l_{3}}{\partial \varepsilon}\right|_{\varepsilon=0}=\left[\frac{H_{x}^{-}(Q(a))}{H_{x}^{+}(Q(a))}-1\right] \int_{\widehat{P Q}} g_{1}^{+} \mathrm{d} y-g_{2}^{+} \mathrm{d} x
$$


Finalmente,

$$
\frac{\partial l_{1}}{\partial \varepsilon}=\frac{\partial}{\partial \varepsilon}\left[H^{+}\left(P^{\varepsilon}\right)-H^{-}\left(P^{\varepsilon}\right)\right]=H_{x}^{+}(p(a, \varepsilon), 0) \frac{\partial}{\partial \varepsilon} p(a, \varepsilon)-H_{x}^{-}(p(a, \varepsilon), 0) \frac{\partial}{\partial \varepsilon} p(a, \varepsilon) .
$$

Então,

$$
\left.\frac{\partial l_{1}}{\partial \varepsilon}\right|_{\varepsilon=0}=\left.\left[H_{x}^{+}(P(a))-H_{x}^{-}(P(a))\right] \frac{\partial}{\partial \varepsilon} p(a, \varepsilon)\right|_{\varepsilon=0} .
$$

De (4.51)

$$
\left.\frac{\partial l_{1}}{\partial \varepsilon}\right|_{\varepsilon=0}=\left[\frac{H_{x}^{+}(P(a))}{H_{x}^{-}(P(a))}-1\right] \int_{\widehat{Q P}} g_{1}^{-} \mathrm{d} y-g_{2}^{-} \mathrm{d} x+\left[\frac{H_{x}^{+}(P(a)) H_{x}^{-}(Q(a))}{H_{x}^{+}(Q(a)) H_{x}^{-}(P(a))}-\frac{H_{x}^{-}(Q(a))}{H_{x}^{+}(Q(a))}\right] \int_{\widehat{P Q}} g_{1}^{+} \mathrm{d} y-g_{2}^{+} \mathrm{d} x .
$$

Temos que

$$
\mathcal{M}(a)=\left.\sum_{j=1}^{4} \frac{\partial l_{j}}{\partial \varepsilon}\right|_{\varepsilon=0} .
$$

A primeira parte fica demonstrada substituindo (4.45), (4.49), (4.53) e (4.55) em (4.56), para obter (4.38). A segunda parte segue diretamente do Lema 4.2.1, O Teorema 4.2.1 está provado.

Exemplo 4.2.1. Considere o sistema (4.32) com campos de vetores descontínuos $F$ e $G$ dados em (4.33) e (4.34) respectivamente definidos por

$$
F^{-}(x, y)=(1,2 x), \quad F^{+}(x, y)=(-1,2 x)
$$

$e$

$$
G^{-}(x, y)=\left(1-y, 1+x+x^{4}\right), \quad G^{+}(x, y)=(1+x, 2 / 3)
$$

Temos que, $H^{+}(x, y)=y+x^{2}$ e $H^{-}(x, y)=-y+x^{2}$ são funções Hamiltonianas por partes do sistema não perturbado. Considere para $y \geq 0$ um arco de solução $\Gamma_{a}^{+}$com origem em $P(a)=(a, 0)$ e extremidade em $Q(a)=(-a, 0)$, onde $a>0$, definido por

$$
H^{+}(x, y)=a^{2}
$$

e para $y \leq 0$ o arco de solução $\Gamma_{a}^{-}$com origem em $Q(a)=(-a, 0)$ e extremidade em $P(a)=(a, 0)$, definido por

$$
H^{-}(x, y)=a^{2}
$$

Neste caso o sistema não perturbado possui uma família a um parâmetro de órbitas periódicas denotadas por

$$
\Gamma_{a}=\Gamma_{a}^{+} \cup \Gamma_{a}^{-}
$$

com $\Gamma_{a}$ parametrizada por $\gamma_{a}$ curva suave por partes. Parametrizemos $\Gamma_{a}^{+}$por

$$
\gamma_{a}^{+}(s)=\left(a \cos (s), a^{2} \operatorname{sen}^{2}(s)\right), \quad s \in[0, \pi]
$$


$e \Gamma_{a}^{-}$parametrizada por

$$
\gamma_{a}^{-}(s)=\left(a \cos (s),-a^{2} \operatorname{sen}^{2}(s)\right), \quad s \in[\pi, 2 \pi] .
$$

Do Teorema 4.2.1 obtemos

$$
\begin{aligned}
\mathcal{M}(a) & =\oint_{\gamma_{a}^{+}} g_{1}^{+} \mathrm{d} y-g_{2}^{+} \mathrm{d} x+\oint_{\gamma_{a}^{-}} g_{1}^{-} \mathrm{d} y-g_{2}^{-} \mathrm{d} x \\
& =\int_{0}^{\pi}\left[g_{1}^{+}\left(\gamma_{a}^{+}(s)\right)\left(2 a^{2} \operatorname{sen}(s) \cos (s)\right)-g_{2}^{+}\left(\gamma_{a}^{+}(s)\right)(-a \operatorname{sen}(s))\right] \mathrm{d} s \\
& +\int_{\pi}^{2 \pi}\left[g_{1}^{-}\left(\gamma_{a}^{-}(s)\right)\left(-2 a^{2} \operatorname{sen}(s) \cos (s)\right)-g_{2}^{-}\left(\gamma_{a}^{-}(s)\right)(-a \operatorname{sen}(s))\right] \mathrm{d} s \\
& =\int_{0}^{\pi}\left[\frac{2}{3} a \operatorname{sen}(s)+(1+a \cos (s))\left(2 a^{2} \operatorname{sen}(s) \cos (s)\right)\right] \mathrm{d} s \\
& +\int_{\pi}^{2 \pi}\left[\left(1+a \cos (s)+a^{4} \cos ^{4}(s)\right)(a \operatorname{sen}(s))-\left(1+a^{2} \operatorname{sen}^{2}(s)\right)\left(2 a^{2} \operatorname{sen}(s) \cos (s)\right)\right] \mathrm{d} s .
\end{aligned}
$$

Fazendo os cálculos obtemos

$$
\mathcal{M}(a)=-\frac{2}{15} a\left(3 a^{4}-10 a^{2}+5\right) .
$$

As raízes positivas de $\mathcal{M}$ são

$$
a_{1}=\sqrt{\frac{1}{3}(5-\sqrt{10})} \quad \text { e } \quad a_{2}=\sqrt{\frac{1}{3}(5+\sqrt{10})} .
$$

Além disso,

$$
\mathcal{M}^{\prime}\left(a_{1}\right)=\frac{8}{9}(-2+\sqrt{10})>0 \quad \text { e } \quad \mathcal{M}^{\prime}\left(a_{2}\right)=-\frac{8}{9}(2+\sqrt{10})<0 .
$$

Portanto para $\varepsilon$ suficientemente pequeno existe um ciclo limite instável $X_{1}^{\varepsilon}$ de (4.32) tal que $X_{1}^{\varepsilon}$ tende a órbita periódica $\Gamma_{a_{1}}$ quando $\varepsilon$ tende a 0 e um ciclo limite estável $X_{2}^{\varepsilon}$ de (4.32) tal que $X_{2}^{\varepsilon}$ tende a órbita periódica $\Gamma_{a_{2}}$ quando $\varepsilon$ tende a 0.

\section{Prova do Teorema 4.2.2}

Começamos a prova do Teorema 4.2.2 analisando a função $\mathcal{R}$ dada em (4.42).

Se $0<s<T^{+}(a, 0)$ então $y_{a}(s)>0$. Pela continuidade da função de regularização $\varphi$ e como $y_{a}(s)$ é limitada temos que

$$
\lim _{\mu \rightarrow 0} \varphi^{\mu}\left(y_{a}(s)\right)=\lim _{\mu \rightarrow 0} \varphi\left(\frac{y_{a}(s)}{\mu}\right)=\varphi\left(\lim _{\mu \rightarrow 0} \frac{y_{a}(s)}{\mu}\right)=1 .
$$

Assim,

$$
\lim _{\mu \rightarrow 0} r_{1}\left(\gamma_{a}(s), \mu\right)=g_{1}^{+}\left(\gamma_{a}(s)\right)
$$




$$
\lim _{\mu \rightarrow 0} r_{2}\left(\gamma_{a}(s), \mu\right)=g_{2}^{+}\left(\gamma_{a}(s)\right)
$$

Agora, se $T^{+}(a, 0)<s<T^{-}(a, 0)$ então $y_{a}(s)<0$. Pela continuidade da função de regularização $\varphi$ e como $y_{a}(s)$ é limitada temos que

$$
\lim _{\mu \rightarrow 0} \varphi^{\mu}\left(y_{a}(s)\right)=\lim _{\mu \rightarrow 0} \varphi\left(\frac{y_{a}(s)}{\mu}\right)=\varphi\left(\lim _{\mu \rightarrow 0} \frac{y_{a}(s)}{\mu}\right)=0 .
$$

Assim,

$$
\lim _{\mu \rightarrow 0} r_{1}\left(\gamma_{a}(s), \mu\right)=g_{1}^{-}\left(\gamma_{a}(s)\right)
$$

$\mathrm{e}$

$$
\left.\lim _{\mu \rightarrow 0} r_{2}\left(\gamma_{a}(s), \mu\right)=g_{2}^{-}\left(\gamma_{a}(s)\right)\right) .
$$

Se $s=0, s=T^{+}(a, 0)$ ou $s=T^{-}(a, 0)$ temos $y_{a}(s)=0$. Assim, $s=0, s=T^{+}(a, 0)$ ou $s=T^{-}(a, 0)$, temos

$$
\lim _{\mu \rightarrow 0} r_{1}\left(\gamma_{a}(s), \mu\right)=g_{1}^{-}\left(x_{a}(s), 0\right)+\varphi(0)\left(g_{1}^{+}\left(x_{a}(s), 0\right)-g_{1}^{-}\left(x_{a}(s), 0\right)\right)
$$

e

$$
\lim _{\mu \rightarrow 0} r_{2}\left(\gamma_{a}(s), \mu\right)=g_{2}^{-}\left(x_{a}(s), 0\right)+\varphi(0)\left(g_{2}^{+}\left(x_{a}(s), 0\right)-g_{2}^{-}\left(x_{a}(s), 0\right)\right) .
$$

Para cada $a \in I$ e $\mu>0$ fixados, defina a função $F:\left[0, T^{+}(a, 0)\right] \rightarrow \mathbb{R}$ por

$$
F^{+}(a, s, \mu)=r_{1}\left(\gamma_{a}(s), \mu\right) \dot{y}_{a}(s)-r_{2}\left(\gamma_{a}(s), \mu\right) \dot{x}_{a}(s) .
$$

Como $r_{1}, r_{2}, \dot{x}_{a}(s)$ e $\dot{y}_{a}(s)$ são funções contínuas em $\left[0, T^{+}(a, 0)\right]$ para todo $a \in I$ e $\mu>0$ fixados, então $F^{+}$é contínua neste intervalo.

Considere a sequência

$$
F_{n}^{+}(a, s)=F^{+}\left(a, s, \mu_{n}\right)
$$

onde $\left(\mu_{n}\right)_{n \in \mathbb{N}}$ é uma sequência de números reais positivos que tende a 0 quando $n$ tende a infinito. Para cada $n \in \mathbb{N}$ a função $\left\{F_{n}^{+}\right\}$é Riemann integrável em $\left[0, T^{+}(a, 0)\right]$. Temos que $F_{n}^{+}(a, s) \rightarrow F_{0}^{+}(a, s)$ quase sempre em $\left[0, T^{+}(a, 0)\right]$ quando $n \rightarrow \infty$, onde

$$
F_{0}^{+}(a, s)=g_{1}^{+}\left(\gamma_{a}(s)\right) \dot{y}_{a}(s)(s)-g_{2}^{+}\left(\gamma_{a}(s)\right) \dot{x}_{a}(s) \text {. }
$$

Além disso, $F_{0}^{+}(a, s)$ é Riemann integrável em $\left[0, T^{+}(a, 0)\right]$. A sequência $\left\{F_{n}^{+}\right\}$é limitada pois suas funções componentes são funções contínuas em $\left[0, T^{+}(a, 0)\right]$ e definidas no conjunto compacto $K=\gamma_{a}^{+}\left(\left[0, T^{+}(a, 0)\right]\right)$, onde $\gamma_{a}^{+}(s)=\left(x_{a}(s), y_{a}(s)\right)$.

Pela análise acima, estamos nas hipóteses do Teorema 4.1.5. Assim,

$$
\lim _{n \rightarrow+\infty} \int_{0}^{T^{+}(a, 0)} F_{n}^{+}(a, s) \mathrm{d} s=\int_{0}^{T^{+}(a, 0)} F_{0}^{+}(a, s) \mathrm{d} s .
$$


Da mesma forma, defina a função $F^{-}:\left[T^{+}(a, 0), T^{-}(a, 0)\right] \rightarrow \mathbb{R}$ dada por

$$
F^{-}(a, s, \mu)=r_{1}\left(\gamma_{a}(s), \mu\right) \dot{y}_{a}(s)-r_{2}\left(\gamma_{a}(s), \mu\right) \dot{x}_{a}(s)
$$

e a sequência

$$
F_{n}^{-}(a, s)=F^{-}\left(a, s, \mu_{n}\right)
$$

onde $\left(\mu_{n}\right)_{n \in \mathbb{N}}$ é uma sequência de números reais positivos que tende a 0 quando $n$ tende a infinito. Com os mesmos argumentos acima, $F_{n}^{-}(a, s) \rightarrow F_{0}^{-}(a, s)$ quase sempre em $\left[T^{+}(a, 0), T^{-}(a, 0)\right]$ quando $n \rightarrow \infty$, onde

$$
F_{0}^{-}(a, s)=g_{1}^{-}\left(\gamma_{a}(s)\right) \dot{y}_{a}(s)(s)-g_{2}^{-}\left(\gamma_{a}(s)\right) \dot{x}_{a}(s) .
$$

Assim, usando novamente o Teorema 4.1.5, obtemos

$$
\lim _{n \rightarrow+\infty} \int_{T^{+}(a, 0)}^{T^{-}(a, 0)} F_{n}^{-}(a, s) \mathrm{d} s=\int_{T^{+}(a, 0)}^{T^{-}(a, 0)} F_{0}^{-}(a, s) \mathrm{d} s .
$$

Portanto, usando o Teorema 4.1.6, (4.57) e (4.58) obtemos

$$
\begin{aligned}
\lim _{\mu \rightarrow 0} \mathcal{R}(a, \mu) & =\lim _{n \rightarrow+\infty} \mathcal{R}\left(a, \mu_{n}\right) \\
& =\lim _{n \rightarrow+\infty} \int_{0}^{T^{+}(a, 0)} F_{n}^{+}(a, s) \mathrm{d} s+\lim _{n \rightarrow+\infty} \int_{T^{+}(a, 0)}^{T^{-}(a, 0)} F_{n}^{-}(a, s) \mathrm{d} s \\
& =\int_{0}^{T^{+}(a, 0)} F_{0}^{+}(a, s) \mathrm{d} s+\int_{T^{+}(a, 0)}^{T^{-}(a, 0)} F_{0}^{-}(a, s) \mathrm{d} s \\
& =\mathcal{M}^{+}(a)+\mathcal{M}^{-}(a)=\mathcal{M}(a) .
\end{aligned}
$$

Portanto, o Teorema 4.2 .2 está provado.

\section{Prova do Teorema 4.2.3}

Para que o campo $Z^{\mu}(X, 0)$ seja Hamiltoniano é necessário que

$$
\operatorname{div} F(x, y, \mu)=\frac{\partial f_{1}}{\partial x}(x, y, \mu)+\frac{\partial f_{2}}{\partial y}(x, y, \mu)=0 .
$$

Temos,

$$
\begin{gathered}
\frac{\partial f_{1}}{\partial x}(x, y, \mu)=-H_{x y}^{-}+\varphi_{\mu}(y)\left(H_{x y}^{-}-H_{x y}^{+}\right) \\
\frac{\partial f_{2}}{\partial y}(x, y, \mu)=H_{x y}^{-}+\varphi_{\mu}^{\prime}(y)\left(H_{x}^{+}-H_{x}^{-}\right)+\varphi_{\mu}(y)\left(H_{x y}^{+}-H_{x y}^{-}\right) .
\end{gathered}
$$

Portanto,

$$
\operatorname{div} F(x, y, \mu)=\varphi_{\mu}^{\prime}(y)\left(H_{x}^{+}-H_{x}^{-}\right)
$$


Como $\varphi_{\mu}^{\prime}(y)$ não é identicamente nula, a condição para o campo $Z_{\mu}(X, 0)$ ser Hamiltoniano é que

$$
H_{x}^{+}(x, y)=H_{x}^{-}(x, y)
$$

Com esta hipótese satisfeita temos

$$
Z_{\mu}(X, 0)=\left(-H_{y}^{-}+\varphi_{\mu}(y)\left(H_{y}^{-}-H_{y}^{+}\right), H_{x}^{-}\right)
$$

ou seja, temos para cada $\mu>0$ o sistema suave

$$
\left\{\begin{array}{l}
\dot{x}=-H_{y}^{-}(x, y)+\varphi_{\mu}(y)\left(H_{y}^{-}-H_{y}^{+}\right)(x, y), \\
\dot{y}=H_{x}^{-} .
\end{array}\right.
$$

Um função Hamiltoniana para este sistema é

$$
\widetilde{H}(x, y, \mu)=H^{-}(x, y)+\int \varphi_{\mu}(y)\left(H_{y}^{+}-H_{y}^{-}\right)(x, y) \mathrm{d} y .
$$

Veja que a função $H_{y}^{+}-H_{y}^{-}$é função de $y$ apenas, pois derivando com repeito a $x$ e usando o fato de que $H_{x}^{+}=H_{x}^{-}$, obtemos

$$
\frac{\partial}{\partial x}\left(H_{y}^{+}-H_{y}^{-}\right)(x, y)=0
$$

Além disso, a função $\varphi_{\mu}(y)\left(H_{y}^{+}-H_{y}^{-}\right)(x, y)$ é contínua, portanto, para cada domínio compacto é integrável em $\mathbb{R}$, sempre é possível obter uma função Hamiltoniana $\widetilde{H}$ para o sistema regularizado. Portanto, o Teorema 4.2 .3 está provado.

Seja $\left(x_{0}, y_{0}\right)$ equilíbrio do sistema (4.60). Com a condição (4.59) temos um Campo Hamiltoniano, para que este campo seja um centro para $\left(x_{0}, y_{0}\right)$, é necessário que o determinante da matriz Hessiana de $\widetilde{H}$ em (4.61) para $\left(x_{0}, y_{0}\right)$ seja não nulo, isto é, Hess $\widetilde{H}\left(x_{0}, y_{0}, \mu\right) \neq 0$, onde,

$$
\operatorname{Hess} \widetilde{H}\left(x_{0}, y_{0}, \mu\right)=\left|\begin{array}{cc}
\widetilde{H}_{x x}\left(x_{0}, y_{0}, \mu\right) & \widetilde{H}_{x y}\left(x_{0}, y_{0}, \mu\right) \\
\widetilde{H}_{x y}\left(x_{0}, y_{0}, \mu\right) & \widetilde{H}_{y y}\left(x_{0}, y_{0}, \mu\right)
\end{array}\right| \text {. }
$$

Exemplo 4.2.2. Considere o sistema (4.32) para $\varepsilon=0$ com campo de vetores descontínuo $F$ dado em (4.33) definido por

$$
F^{-}(x, y)=(-1,2 x), \quad F^{+}(x, y)=(1,2 x)
$$

O campo regularizado é dado por

$$
Z^{\mu}(X, 0)=\left(1-2 \varphi^{\mu}(y), 2 x\right)
$$

ou seja, temos para cada $\mu>0$ o sistema suave

$$
\left\{\begin{array}{l}
\dot{x}=1-2 \varphi^{\mu}(y) \\
\dot{y}=2 x
\end{array}\right.
$$


Os equlíbrios deste sistema são dados por $\left(x_{0}, y_{0}\right)$ onde $x_{0}=0$ e $y_{0}=\left(\varphi^{\mu}\right)^{-1}(1 / 2)$ e uma função Hamiltoniana para este sistema é

$$
\widetilde{H}(x, y, \mu)=x^{2}-y+2 \int \varphi^{\mu}(y) \mathrm{d} y .
$$

A Hessiana de $\widetilde{H}$ em $\left(x_{0}, y_{0}\right)$ é dada por

$$
\operatorname{Hess} \widetilde{H}\left(x_{0}, y_{0}, \mu\right)=\left|\begin{array}{cc}
2 & 0 \\
0 & 2\left(\varphi_{\mu}\right)^{\prime}(y)
\end{array}\right|=4\left(\varphi_{\mu}\right)^{\prime}(y)>0 .
$$

Portanto, o sistema (4.62) tem um centro Hamiltoniano para $\left(x_{0}, y_{0}\right)$.

\subsection{Sistema Descontínuo com um Centro Perturbado por Partes}

Considere a função $\mathcal{H}: U \rightarrow \mathbb{R}, U$ aberto do $\mathbb{R}^{2}$, com $\mathcal{H} \in C^{k}(U, \mathbb{R}), k \geq 1$ e os conjuntos $\Sigma^{-}, \Sigma^{+}$e $\Sigma$ definidos da seguinte forma:

$$
\Sigma^{-}=\left\{X \in \mathbb{R}^{2}: \mathcal{H}(X)<0\right\}, \quad \Sigma^{+}=\left\{X \in \mathbb{R}^{2}: \mathcal{H}(X)>0\right\}
$$

e

$$
\Sigma=\left\{X \in \mathbb{R}^{2}: \mathcal{H}(X)=0\right\}
$$

Sejam as funções

$$
F(X)= \begin{cases}F^{-}(X), & X \in \Sigma^{-} \\ F^{+}(X), & X \in \Sigma^{+}\end{cases}
$$

e

$$
G(X)= \begin{cases}G^{-}(X), & X \in \Sigma^{-} \\ G^{+}(X), & X \in \Sigma^{+}\end{cases}
$$

onde $F^{ \pm}: U \rightarrow \mathbb{R}^{2}, G^{ \pm}: U \rightarrow \mathbb{R}^{2}$ campos vetorias suaves de classe $C^{k}, k \geq 1$ e $\varepsilon \in \mathbb{R}$. Considere os sistemas definidos por partes no plano

$$
\dot{X}=F(X)
$$

e

$$
\dot{X}=F(X)+\varepsilon G(X)
$$

Considere as seguintes hipóteses:

(a) A normal à curva $\Sigma$, dada por $N(X)=[D \mathcal{H}(X)]^{T}=\nabla \mathcal{H}(\mathcal{X})$, é não nula em $\Sigma$. 
(b) O sistema (4.63) tem um anel $\mathcal{A}$ de órbitas periódicas, que intercepta $\Sigma$ transversalmente. Além disso, sem perda de generalidade, suponhamos que as órbitas periódicas tenham orientação anti-horária.

Seja uma solução do sistema perturbado (4.64) denotada por $X\left(t, X_{0}, \varepsilon\right)$ que satisfaz a condição inicial $X\left(t_{0}\right)=X\left(t_{0}, X_{0}, \varepsilon\right)=X_{0} \mathrm{com}$

$$
X\left(t, X_{0}, \varepsilon\right)=\left\{\begin{array}{l}
X^{-}\left(t, X_{0}, \varepsilon\right), \quad t_{0} \leq t \leq t_{\Sigma} \\
X^{+}\left(t, X^{-}\left(t_{\Sigma}, X_{0}, \varepsilon\right), \varepsilon\right), \quad t_{\Sigma} \leq t \leq t_{\Sigma}+\theta
\end{array}\right.
$$

para $\theta>0$ suficientemente pequeno, onde $t_{\Sigma}>0$ é tal que $X^{-}\left(t_{\Sigma}, X_{0}, \varepsilon\right) \in \Sigma$ e $X^{-}\left(t, X_{0}, \varepsilon\right)$ é solução de

$$
\dot{X}=F^{-}(X)+\varepsilon G^{-}(X), \quad X\left(t_{0}\right)=X\left(t_{0}, X_{0}, \varepsilon\right)=X_{0},
$$

e $X^{+}\left(t, X^{-}\left(t_{\Sigma}, X_{0}, \varepsilon\right), \varepsilon\right)$ é uma solução de

$$
\dot{X}=F^{+}(X)+\varepsilon G^{+}(X), \quad X\left(t_{\Sigma}\right)=X\left(t_{\Sigma}, X_{0}, \varepsilon\right)=X_{\Sigma} .
$$

Seja $X\left(t, X_{0}, 0\right)$ uma solução periódica de (4.63) com condição inicial $X\left(t_{0}\right)=X_{0}$. Segue da dependência contínua das soluções com repeito aos parâmetros e condições iniciais que, para $\varepsilon$ suficientemente pequeno, uma solução periódica $X(t, Y, \varepsilon)$ do sistema (4.64) também intercepta $\Sigma$ transversalmente para $Y$ suficientemente próximo de $X_{0}$. Ver Observação 4.1.1.

Teorema 4.3.1. Seja $X\left(t, X_{0}, \varepsilon\right)$ solução do sistema (4.64) que intercepta $\Sigma$ transversalmente quando $t=t_{\Sigma}, t_{0}<t_{\Sigma}$ e $X\left(t, X_{0}, \varepsilon\right) \in \Sigma^{-}$para todo $t \in\left(t_{0}, t_{\Sigma}\right)$. Então existem $r>0 e$ $\theta>0$ tal que para todo ponto $Y \in B_{r}\left(X_{0}\right), \tau \in\left(t_{0}-\theta, t_{0}+\theta\right)$ e $\varepsilon$ suficientemente pequeno no intervalo $\left(-\varepsilon_{0}, \varepsilon_{0}\right)$, existe uma solução $X(t, Y, \varepsilon)$ do sistema (4.64) com condição inicial $X(\tau, Y, \varepsilon)=X(\tau)=Y$, que intercepta $\Sigma$ transversalmente quando $t=T(\tau, Y, \varepsilon)$, além dissso, a função $T(\tau, Y, \varepsilon):\left(t_{0}-\theta, t_{0}+\theta\right) \times B_{r}\left(X_{0}\right) \times\left(-\varepsilon_{0}, \varepsilon_{0}\right) \rightarrow \mathbb{R}$, é de classe $C^{k}, k \geq 1$.

Demonstração: Ver [20].

Teorema 4.3.2. Seja $X\left(t, X_{0}, \varepsilon\right)$ solução do sistema (4.64) com condição inicial $X_{0} \in \Sigma^{-}$que intercepta $\Sigma$ transversalmente para $t=t_{\Sigma}=T_{1}\left(t_{0}, X_{0}, \varepsilon\right)$, então $D_{2} X^{-}\left(t, X_{0}, \varepsilon\right)$ é uma matriz fundamental de soluções de

$$
\dot{W}=D F^{-}\left(X^{-}\left(t, X_{0}, \varepsilon\right)\right) W, \quad t_{0} \leq t \leq t_{\Sigma}
$$

e $D_{2} X^{+}\left(t, X^{-}\left(t_{\Sigma}, X_{0}, \varepsilon\right), \varepsilon\right)$ é uma matriz fundamental de soluções de

$$
\dot{W}=D F^{+}\left(X^{+}\left(t, X^{-}\left(t_{\Sigma}, X_{0}, \varepsilon\right), \varepsilon\right)\right) W, \quad t_{\Sigma} \leq t \leq t_{\Sigma}+\theta
$$

onde $D_{2}\left[X^{ \pm}\left(t, X_{0}, \varepsilon\right)\right]=\left.\frac{\partial}{\partial X} X^{ \pm}(t, X, \varepsilon)\right|_{X=X_{0}}$ e D $F^{ \pm}$denota a derivada de $F^{ \pm}$. 
Além disso, para $t=t_{\Sigma}$, estas matrizes estão relacionadas da seguinte forma:

$$
D_{2} X^{+}\left(t_{\Sigma}, X^{-}\left(t_{\Sigma}, X_{0}, \varepsilon\right), \varepsilon\right)=S_{1}\left(X_{0}, \varepsilon\right) D_{2} X^{-}\left(t_{\Sigma}, X_{0}, \varepsilon\right)
$$

onde

$$
S_{1}\left(X_{0}, \varepsilon\right)=I+\frac{\left[F^{+}\left(X_{\Sigma}\right)-F^{-}\left(X_{\Sigma}\right)\right]\left[N^{T}\left(X_{\Sigma}\right)\right]}{N^{T}\left(X_{\Sigma}\right) F^{-}\left(X_{\Sigma}\right)}
$$

com $X_{\Sigma}:=X^{-}\left(t_{\Sigma}, X_{0}, \varepsilon\right)$ e I a matriz identidade.

Demonstração: Ver [20].

O Teorema 4.3 .2 nos ajuda a definir a matriz

$$
D_{2} X\left(t, X_{0}, \varepsilon\right)=\left\{\begin{array}{l}
D_{2} X^{-}\left(t, X_{0}, \varepsilon\right), \quad t_{0} \leq t \leq t_{\Sigma} \\
D_{2} X^{+}\left(t, X^{-}\left(t_{\Sigma}, X_{0}, \varepsilon\right), \varepsilon\right), \quad t_{\Sigma} \leq t \leq t_{\Sigma}+\theta,
\end{array}\right.
$$

chamada de matriz fundamental das soluções do sistema de equações variacionais

$$
\dot{W}=\left\{\begin{array}{l}
D F^{-}\left[X^{-}\left(t, X_{0}, \varepsilon\right)\right] W, \quad t_{0} \leq t \leq t_{\Sigma} \\
D F^{+}\left[X^{+}\left(t, X^{-}\left(t_{\Sigma}, X_{0}, \varepsilon\right), \varepsilon\right)\right] W, \quad t_{\Sigma} \leq t \leq t_{\Sigma}+\theta .
\end{array}\right.
$$

\subsubsection{Extensões do Teorema de Diliberto e Lema Variacional}

Considere uma seção $\widetilde{\Sigma}$ que intercepta transversalmente uma órbita periódica $\Gamma$ do sistema não perturbado (4.63) em $X_{0}$. Suponha que $\Gamma$ é parametrizada por $\gamma$ de período $T_{3}$ e intercepta $\Sigma$ após dois tempos $T_{1}$ e $T_{2}$ tal que $0<T_{1}<T_{2}<T_{3}$.

Seja $X\left(t, X_{0}, 0\right)$ solução do sistema não perturbado (4.63) com $X_{0}=X(0)$ e suponha que $X\left(t, X_{0}, 0\right) \in \Sigma^{-}$para todo $t \in\left(0, T_{1}\right), X\left(t, X_{0}, 0\right) \in \Sigma^{+}$para todo $t \in\left(T_{1}, T_{2}\right)$ e $X\left(t, X_{0}, 0\right) \in \Sigma^{-}$ para todo $t \in\left(T_{2}, T_{3}\right)$.

Denote por $\gamma^{\varepsilon}$ uma solução do sistema perturbado (4.64). Agora, como $\gamma$ intercepta $\widetilde{\Sigma}$ e $\Sigma$ transversalmente, usando o Teorema da Função Implícita e o Teorema 4.3.1, existem funções $\tau_{1}$, $\tau_{2}$ e $\tau_{3}$ tais que

$$
\tau_{1}: \widetilde{\Sigma}_{1} \times\left(-\varepsilon_{0}, \varepsilon_{0}\right) \rightarrow \Sigma
$$

é o tempo para $\gamma^{\varepsilon}$ interceptar $\Sigma$ partindo de $\widetilde{\Sigma}_{1} \subseteq \widetilde{\Sigma} \operatorname{com} \tau_{1}\left(X_{0}, 0\right)=T_{1}$,

$$
\tau_{2}: \widetilde{\Sigma}_{1} \times\left(-\varepsilon_{0}, \varepsilon_{0}\right) \rightarrow \Sigma_{2}
$$

é o tempo para $\gamma^{\varepsilon}$ interceptar $\Sigma_{2} \subseteq \Sigma$ partindo de $\widetilde{\Sigma}_{1} \operatorname{com} \tau_{2}\left(X_{0}, 0\right)=T_{2}$,

$$
\tau_{3}: \widetilde{\Sigma}_{1} \times\left(-\varepsilon_{0}, \varepsilon_{0}\right) \rightarrow \widetilde{\Sigma}_{1}
$$

é o tempo para $\gamma^{\varepsilon}$ interceptar $\widetilde{\Sigma}_{1}$ partindo de $\widetilde{\Sigma}_{1} \operatorname{com} \tau_{3}\left(X_{0}, 0\right)=T_{3}$.

Pela definição da matriz fundamental de soluções, esta pode ser estendida para $T_{2} \leq t \leq T_{3}$. Defina $\gamma$ como sendo $X\left(t, X_{0}, 0\right)=X^{-}\left(t, X^{+}\left(T_{2}, X^{-}\left(T_{1}, X_{0}, 0\right), 0\right), 0\right)$. Segue que, para $t=T_{3}$, $\gamma$ intercepta $\widetilde{\Sigma}_{1}$ novamente em $X_{0}$. 
Teorema 4.3.3. Nas hipóteses acima, seja $\gamma$ dada por $X\left(t, X_{0}, 0\right)$. Então

$$
D_{2} X^{-}\left(t, X^{+}\left(T_{2}, X^{-}\left(T_{1}, X_{0}, 0\right), 0\right), 0\right)
$$

para $T_{2} \leq t \leq T_{3}$ é a matriz fundamental de soluções de

$$
\dot{W}=D F^{-}\left[X^{-}\left(t, X^{+}\left(T_{2}, X^{-}\left(T_{1}, X_{0}, 0\right), 0\right), 0\right)\right] W
$$

e relacionada com (4.67) por

$$
\left.D_{2} X^{-}\left(T_{2}, \tilde{b}\left(X_{0}, 0\right), 0\right)=S_{2}\left(X_{0}, 0\right) D_{2} \tilde{b}\left(X_{0}, 0\right) S_{1}\left(X_{0}, 0\right) D_{2} \tilde{a}\left(X_{0}, 0\right)\right]
$$

onde

$$
\begin{gathered}
\tilde{b}\left(X_{0}, 0\right):=X^{+}\left(T_{2}, X^{-}\left(T_{1}, X_{0}, 0\right), 0\right), \\
\tilde{a}\left(X_{0}, 0\right):=X^{-}\left(T_{1}, X_{0}, 0\right), \\
S_{2}\left(X_{0}, 0\right):=I+\frac{\left[F^{-}\left(\tilde{b}\left(X_{0}, 0\right)\right)-F^{+}\left(\tilde{b}\left(X_{0}, 0\right)\right)\right]\left[N^{T}\left(\tilde{b}\left(X_{0}, 0\right)\right)\right]}{N^{T}\left(\tilde{b}\left(X_{0}, 0\right)\right) F^{+}\left(\tilde{b}\left(X_{0}, 0\right)\right)}
\end{gathered}
$$

e $S_{1}\left(X_{0}, 0\right)$ é dado em (4.66) tomando $t_{\Sigma}=T_{1}$ e $\varepsilon=0$.

Demonstração: Seja $\varepsilon$ suficientemente pequeno, $Y \in \widetilde{\Sigma}_{1}$ numa vizinhança suficientemente pequena de $X_{0}$ e $t \in\left(\tau_{2}(Y, \varepsilon), \tau_{2}(Y, \varepsilon)+\theta\right)$. Da dependência contínua das soluções com respeito aos parâmetros e condições iniciais segue que a aplicação $t \mapsto X^{-}(t, \tilde{b}(Y, \varepsilon), \varepsilon)$ é contínua onde $\tilde{b}(Y, \varepsilon)=X^{+}\left(\tau_{2}(Y, \varepsilon), X^{-}\left(\tau_{1}(Y, \varepsilon), Y, \varepsilon\right), \varepsilon\right)$ e $X^{-}(t, \tilde{b}(Y, \varepsilon), \varepsilon)$ é a única solução de

$$
\dot{X}=F^{-}(X)+\varepsilon G^{-}(X), \quad X\left(\tau_{2}(Y, \varepsilon)\right)=X\left(\tau_{2}(Y, \varepsilon), Y, \varepsilon\right)=\tilde{b}(Y, \varepsilon) .
$$

Do Teorema Fundamental do Cálculo segue que

$$
X^{-}(t, \tilde{b}(Y, \varepsilon), \varepsilon)=\tilde{b}(Y, \varepsilon)+\int_{\tau_{2}(Y, \varepsilon)}^{t}\left[F^{-}\left(X^{-}(s, \tilde{b}(Y, \varepsilon), \varepsilon)\right)+\varepsilon G^{-}\left(X^{-}(s, \tilde{b}(Y, \varepsilon), \varepsilon)\right)\right] \mathrm{d} s .
$$

Além disso, segue da dependência diferenciável das soluções com respeito aos parâmetros e condições iniciais que (4.69) é diferenciável com respeito a $Y$. Derivando (4.69) com respeito a $Y$ e avaliando em $Y=X_{0}$ e $\varepsilon=0$, obtemos

$$
\begin{aligned}
D_{2} X^{-}\left(t, \tilde{b}\left(X_{0}, 0\right), 0\right) & =D_{2} \tilde{b}\left(X_{0}, 0\right)+F^{-}\left(X^{-}\left(T_{2}, \tilde{b}\left(X_{0}, 0\right), 0\right)\right) \cdot D_{1} \tau_{2}\left(X_{0}, 0\right) \\
& +\int_{T_{2}}^{t} D F^{-}\left[X^{-}\left(s, \tilde{b}\left(X_{0}, 0\right), 0\right)\right] D_{2} X^{-}\left(s, \tilde{b}\left(X_{0}, 0\right), 0\right) \mathrm{d} s .
\end{aligned}
$$

Veja que $X^{-}\left(\tau_{1}(Y, \varepsilon), Y, \varepsilon\right) \in \Sigma$ e $X^{+}\left(\tau_{2}(Y, \varepsilon), X^{-}\left(\tau_{1}(Y, \varepsilon), Y, \varepsilon\right), \varepsilon\right) \in \Sigma$, então

$$
\mathcal{H}\left(X^{-}\left(\tau_{1}(Y, \varepsilon), Y, \varepsilon\right)\right)=0
$$

e

$$
\mathcal{H}\left(X^{+}\left(\tau_{2}(Y, \varepsilon), X^{-}\left(\tau_{1}(Y, \varepsilon), Y, \varepsilon\right), \varepsilon\right)\right)=0 .
$$


Derivando (4.71) e (4.72) com respeito a $Y$ e avaliando em $Y=X_{0}$ e $\varepsilon=0$, obtemos

$$
D_{1} \tau_{1}\left(X_{0}, 0\right)=-\frac{N^{T}\left(X_{1 \Sigma}\right) D_{2} X^{-}\left(T_{1}, X_{0}, 0\right)}{N^{T}\left(X_{1 \Sigma}\right) \cdot F^{-}\left(X_{1 \Sigma}\right)}
$$

$\operatorname{com} X_{1 \Sigma}=X^{-}\left(T_{1}, X_{0}, 0\right), \mathrm{e}$

$$
\begin{aligned}
D_{1} \tau_{2}\left(X_{0}, 0\right) & =-\frac{N^{T}\left(X_{2 \Sigma}\right) \cdot\left[D_{2} \tilde{b}\left(X_{0}, 0\right) \cdot D_{2} \tilde{a}\left(X_{0}, 0\right)+D_{2} \tilde{b}\left(X_{0}, 0\right) \cdot F^{-}\left(\tilde{a}\left(X_{0}, 0\right)\right) \cdot D_{1} \tau_{1}\left(X_{0}, 0\right)\right]}{N^{T}\left(X_{2 \Sigma}\right) \cdot F^{+}\left(X_{2 \Sigma}\right)} \\
& +\frac{-D_{2} \tilde{b}\left(X_{0}, 0\right) \cdot F^{+}\left(\tilde{a}\left(X_{0}, 0\right)\right) \cdot D_{1} \tau_{1}\left(X_{0}, 0\right)}{N^{T}\left(X_{2 \Sigma}\right) \cdot F^{+}\left(X_{2 \Sigma}\right)}
\end{aligned}
$$

$\operatorname{com} X_{2 \Sigma}=X^{+}\left(T_{2}, X^{-}\left(T_{1}\left(X_{0}, 0\right), X_{0}, 0\right), 0\right)$.

Agora, por (4.70), obtemos

$$
\begin{aligned}
D_{2} X^{-}\left(t, \tilde{b}\left(X_{0}, 0\right), 0\right) & =S_{2}\left(X_{0}, 0\right) \cdot D_{2} \tilde{b}\left(X_{0}, 0\right)+S_{1}\left(X_{0}, 0\right) \cdot D_{2} X^{-}\left(T_{1}, X_{0}, 0\right) \\
& +\int_{T_{2}}^{t} D F^{-}\left[X^{-}\left(s, \tilde{b}\left(X_{0}, 0\right), 0\right)\right] D_{2} X^{-}\left(s, \tilde{b}\left(X_{0}, 0\right), 0\right) \mathrm{d} s
\end{aligned}
$$

Substituindo $t=T_{2}$ em (4.73) obtemos (4.68).

Observação 4.3.1. Nas regiões (zonas) $\Sigma^{-} e \Sigma^{+}$o sistema (4.64) é suave, logo podemos aplicar o Teorema de Diliberto 2.1.6 dado na Seção 2.1. Mas em $\Sigma$, isto é, na curva $\mathcal{H}(X)=0$ o sistema (4.64) não é contínuo. Portanto, não podemos aplicar diretamente o Teorema de Diliberto. Neste caso como as órbitas interceptam $\Sigma$ transversalmente temos

$$
\Phi\left(t_{\Sigma}\right)=\Phi\left(t_{\Sigma}^{-}\right) S \Phi\left(t_{\Sigma}^{+}\right)
$$

onde $t_{\Sigma}$ é o tempo para interseção de uma solução com $\Sigma$,

$$
\Phi\left(t_{\Sigma}^{-}\right)=\lim _{t \rightarrow t_{\Sigma}^{-}} \Phi(t), \Phi\left(t_{\Sigma}^{+}\right)=\lim _{t \rightarrow t_{\Sigma}^{+}} \Phi(t),
$$

onde $\Phi(t)$ é matriz fundamental de soluções e $S$ é chamada matriz de saltação. Para mais informações ver [23]. Para $t<t_{\Sigma}$, as órbitas estão $\Sigma^{-}$e para $t>t_{\Sigma}$, estão em $\Sigma^{+}$. Assim, podemos aplicar o Teorema de Diliberto em $\Sigma$ usando $\Phi\left(t_{\Sigma}^{-}\right)$e $\Phi\left(t_{\Sigma}^{+}\right)$. Portanto, o Teorema de Diliberto pode ser estendido em toda região.

Teorema 4.3.4. (Extensão do Teorema de Diliberto) Suponha que $X(t, Y, 0)$ é solução do sistema (4.64), para $\varepsilon=0$, isto é, de $\dot{X}=F(X)$ e que satisfaz $X(0, Y, 0)=Y$. Para $Y \in \Sigma^{-}$se $F(Y) \neq 0$, então existe uma matriz fundamental de soluções $\Phi(r, t), r \in\left\{0, T_{1}, T_{2}\right\}$ para a equação variacional $\dot{W}=D F(X(t, Y, 0)) W$, tal que para $0 \leq t \leq T_{1}$

$$
\begin{aligned}
& \Phi(0, t) F^{-}(Y)=F^{-}\left(X^{-}(t, Y, 0)\right), \\
& \Phi(0, t)\left(F^{-}\right)^{\perp}(Y)=a^{-}(0, t, Y) F^{-}\left(X^{-}(t, Y, 0)\right)+b^{-}(0, t, Y)\left(F^{-}\right)^{\perp}\left(X^{-}(t, Y, 0)\right) .
\end{aligned}
$$


Para $T_{1} \leq t \leq T_{2}$,

$$
\begin{aligned}
\Phi\left(T_{1}, t\right) F^{+}\left(X^{-}\left(T_{1}, Y, 0\right)\right)=F^{+} & \left(X^{+}\left(t, X^{-}\left(T_{1}, Y, 0\right), 0\right)\right), \\
\Phi\left(T_{1}, t\right)\left(F^{+}\right)^{\perp}\left(X^{-}\left(T_{1}, Y, 0\right)\right)= & a^{+}\left(T_{1}, t, X^{-}\left(T_{1}, Y, 0\right)\right) F^{+}\left(X^{+}\left(t, X^{-}\left(T_{1}, Y, 0\right), 0\right)\right) \\
& +b^{+}\left(T_{1}, t, X^{-}\left(T_{1}, Y, 0\right)\right)\left(F^{+}\right)^{\perp}\left(X^{+}\left(t, X^{-}\left(T_{1}, Y, 0\right), 0\right)\right) .
\end{aligned}
$$

Para $T_{2} \leq t \leq T_{3}$

$$
\begin{aligned}
& \Phi\left(T_{2}, t\right) F^{-}\left(X^{+}\left(T_{2}, X^{-}\left(T_{1}, Y, 0\right), 0\right)\right)=F^{-}\left(X^{-}\left(t, X^{+}\left(T_{2}, X^{-}\left(T_{1}, Y, 0\right), 0\right), 0\right)\right), \\
& \Phi\left(T_{2}, t\right)\left(F^{-}\right)^{\perp}\left(X^{+}\left(T_{2}, X^{-}\left(T_{1}, Y, 0,0\right)\right)\right)= \\
& a^{-}\left(T_{2}, t, X^{+}\left(T_{2}, X^{-}\left(T_{1}, Y, 0\right), 0\right)\right) F^{-}\left(X^{-}\left(t, X^{+}\left(T_{2}, X^{-}\left(T_{1}, Y, 0\right), 0\right), 0\right)\right) \\
& +b^{-}\left(T_{2}, t, X^{+}\left(T_{2}, X^{-}\left(T_{1}, Y, 0\right), 0\right)\right)\left(F^{-}\right)^{\perp}\left(X^{-}\left(t, X^{+}\left(T_{2}, X^{-}\left(T_{1}, Y, 0\right), 0\right), 0\right)\right),
\end{aligned}
$$

onde

$$
\begin{gathered}
b^{ \pm}(r, t, Y)=\frac{\left\|F^{ \pm}(Y)\right\|^{2}}{\left\|F^{ \pm}\left(X^{ \pm}(t, Y, 0)\right)\right\|^{2}} \cdot \exp \left(\int_{r}^{t} \operatorname{div} F^{ \pm}\left(X^{ \pm}(s, Y, 0)\right), \mathrm{d} s\right) \\
a^{ \pm}(r, t, Y)=\int_{r}^{t}\left[2 \kappa^{ \pm}(s, Y)\left\|F^{ \pm}\left(X^{ \pm}(s, Y, 0)\right)\right\|-\operatorname{curl} F^{ \pm}\left(X^{ \pm}(s, Y, 0)\right)\right] \cdot b^{ \pm}(s, r, Y) \mathrm{d} s .
\end{gathered}
$$

Demonstração: Em (4.67) tome $t_{0}=0, t_{\Sigma}=T_{1}$ e $t_{\Sigma}+\theta=T_{2}$, juntamente com o Teorema 4.3 .3 obtemos a matriz fundamental de soluções

$$
D_{2} X(t, Y, 0)=\left\{\begin{array}{l}
D_{2} X^{-}(t, Y, 0), \quad 0 \leq t \leq T_{1} \\
D_{2} X^{+}\left(t, X^{-}\left(T_{1}, Y, 0\right), 0\right), \quad T_{1} \leq t \leq T_{2} \\
D_{2} X^{-}\left(t, X^{+}\left(T_{2}, X^{-}\left(T_{1}, Y, 0\right), 0\right), 0\right), \quad T_{2} \leq t \leq T_{3}
\end{array}\right.
$$

para a equação variacional

$$
\dot{W}=\left\{\begin{array}{l}
D F^{-}\left[X^{-}(t, Y, 0)\right] W, \quad 0 \leq t \leq T_{1} \\
D F^{+}\left[X^{+}\left(t, X^{-}\left(T_{1}, Y, 0\right), 0\right)\right] W, \quad T_{1} \leq t \leq T_{2} \\
D F^{+}\left[X^{-}\left(t, X^{+}\left(T_{2}, X^{-}\left(T_{1}, Y, 0\right), 0\right), 0\right)\right] W, \quad T_{2} \leq t \leq T_{3} .
\end{array}\right.
$$

Como $X^{-}\left(T_{1}, Y, 0\right)$ e $X^{+}\left(T_{2}, X^{-}\left(T_{1}, Y, 0\right), 0\right)$ não são equilíbrios, temos que, $F^{ \pm}$é não nulo nestes pontos. Portanto, podemos aplicar o Teorema de Diliberto 2.1.6 em cada intervalo.

Teorema 4.3.5. (Extensão do Lema Variacional)

Seja $X\left(t, X_{0}, \varepsilon\right)$ solução do sistema (4.64) onde $X_{0}=X(0)$. Então

$$
\begin{aligned}
\left.\frac{\partial}{\partial \varepsilon} X^{-}\left(t, X_{0}, \varepsilon\right)\right|_{\varepsilon=0, t=T_{1}} & =\left(\mathcal{N}_{1}\left(X_{0}\right)+a^{-}\left(0, T_{1}, X_{0}\right) \mathcal{M}_{1}\left(X_{0}\right)\right) \cdot F^{-}\left(X_{0}\right) \\
& +b^{-}\left(0, T_{1}, X_{0}\right) \cdot \mathcal{M}_{1}\left(X_{0}\right) \cdot\left(F^{-}\right)^{\perp}\left(X_{0}\right) \\
\left.\frac{\partial}{\partial \varepsilon} X^{+}\left(t, X_{1 \Sigma}, \varepsilon\right)\right|_{\varepsilon=0, t=T_{2}} & =\left(\mathcal{N}_{2}\left(X_{0}\right)+a^{+}\left(T_{1}, T_{2}, X_{1 \Sigma}\right) \mathcal{M}_{2}\left(X_{0}\right)\right) \cdot F^{+}\left(X_{1 \Sigma}\right) \\
& +b^{+}\left(T_{1}, T_{2}, X_{1 \Sigma}\right) \cdot \mathcal{M}_{2}\left(X_{0}\right) \cdot\left(F^{+}\right)^{\perp}\left(X_{1 \Sigma}\right)
\end{aligned}
$$




$$
\begin{aligned}
\left.\frac{\partial}{\partial \varepsilon} X^{-}\left(t, X_{2 \Sigma}, \varepsilon\right)\right|_{\varepsilon=0, t=T_{3}} & =\left(\mathcal{N}_{3}\left(X_{0}\right)+a^{-}\left(T_{2}, T_{3}, X_{2 \Sigma}\right) \mathcal{M}_{3}\left(X_{0}\right)\right) \cdot F^{-}\left(X_{2 \Sigma}\right) \\
& +b^{-}\left(T_{2}, T_{3}, X_{2 \Sigma}\right) \cdot \mathcal{M}_{3}\left(X_{0}\right) \cdot\left(F^{-}\right)^{\perp}\left(X_{2 \Sigma}\right)
\end{aligned}
$$

onde

$$
\begin{aligned}
& \mathcal{M}_{1}\left(X_{0}\right)=\int_{0}^{T_{1}} \frac{F^{-}\left(X^{-}\left(t, X_{0}, 0\right)\right) \wedge G^{-}\left(X^{-}\left(t, X_{0}, 0\right)\right)}{b^{-}\left(0, t, X_{0}\right)\left\|F^{-}\left(X^{-}\left(t, X_{0}, 0\right)\right)\right\|^{2}} \mathrm{~d} t, \\
& \mathcal{N}_{1}\left(X_{0}\right)=\int_{0}^{T_{1}} \frac{\left\langle G^{-}\left(X^{-}\left(t, X_{0}, 0\right)\right) F^{-}\left(X^{-}\left(t, X_{0}, 0\right)\right)\right\rangle}{\left\|F^{-}\left(X^{-}\left(t, X_{0}, 0\right)\right)\right\|^{2}} \mathrm{~d} t \\
& -\int_{0}^{T_{1}} \frac{a^{-}\left(0, t, X_{0}\right) \cdot\left(F^{-}\left(X^{-}\left(t, X_{0}, 0\right)\right) \wedge G^{-}\left(X^{-}\left(t, X_{0}, 0\right)\right)\right)}{b^{-}\left(0, t, X_{0}\right)\left\|F^{-}\left(X^{-}\left(t, X_{0}, 0\right)\right)\right\|^{2}} \mathrm{~d} t, \\
& \mathcal{M}_{2}\left(X_{0}\right)=\int_{T_{1}}^{T_{2}} \frac{F^{+}\left(X^{+}\left(t, \tilde{a}\left(X_{0}, 0\right), 0\right)\right) \wedge G^{+}\left(X^{+}\left(t, \tilde{a}\left(X_{0}, 0\right), 0\right)\right)}{b^{+}\left(T_{1}, t, \tilde{a}\left(X_{0}, 0\right)\right)\left\|F^{+}\left(X^{+}\left(t, \tilde{a}\left(X_{0}, 0\right), 0\right)\right)\right\|^{2}} \mathrm{~d} t, \\
& \mathcal{N}_{2}\left(X_{0}\right)=\int_{T_{1}}^{T_{2}} \frac{\left\langle F^{+}\left(X^{+}\left(t, \tilde{a}\left(X_{0}, 0\right), 0\right)\right), G^{+}\left(X^{+}\left(t, \tilde{a}\left(X_{0}, 0\right), 0\right)\right)\right\rangle}{\left\|F^{+}\left(X^{+}\left(t, \tilde{a}\left(X_{0}, 0\right), 0\right)\right)\right\|^{2}} \mathrm{~d} t \\
& -\int_{T_{1}}^{T_{2}} \frac{a^{+}\left(T_{1}, t, \tilde{a}\left(X_{0}, 0\right)\right) \cdot\left(F^{+}\left(X^{+}\left(t, \tilde{a}\left(X_{0}, 0\right), 0\right)\right) \wedge G^{+}\left(X^{+}\left(t, \tilde{a}\left(X_{0}, 0\right), 0\right)\right)\right)}{b^{+}\left(T_{1}, t, \tilde{a}\left(X_{0}, 0\right)\right)\left\|F^{+}\left(X^{+}\left(t, \tilde{a}\left(X_{0}, 0\right), 0\right)\right)\right\|^{2}} \mathrm{~d} t, \\
& \mathcal{M}_{3}\left(X_{0}\right)=\int_{T_{2}}^{T_{3}} \frac{F^{-}\left(X^{-}\left(t, \tilde{b}\left(X_{0}, 0\right), 0\right)\right) \wedge G^{-}\left(X^{-}\left(t, \tilde{b}\left(X_{0}, 0\right), 0\right)\right)}{b^{-}\left(T_{2}, t, \tilde{b}\left(X_{0}, 0\right)\right)\left\|F^{-}\left(X^{-}\left(t, \tilde{b}\left(X_{0}, 0\right), 0\right)\right)\right\|^{2}} \mathrm{~d} t \\
& \mathcal{N}_{3}\left(X_{0}\right)=\int_{T_{2}}^{T_{3}} \frac{\left\langle F^{-}\left(X^{-}\left(t, \tilde{b}\left(X_{0}, 0\right), 0\right)\right), G^{-}\left(X^{-}\left(t, \tilde{b}\left(X_{0}, 0\right), 0\right)\right)\right\rangle}{\left\|F^{-}\left(X^{-}\left(t, \tilde{b}\left(X_{0}, 0\right), 0\right)\right)\right\|^{2}} \mathrm{~d} t \\
& -\int_{T_{2}}^{T_{3}} \frac{a^{-}\left(T_{1}, t, \tilde{b}\left(X_{0}, 0\right)\right) \cdot\left(F^{-}\left(X^{-}\left(t, \tilde{b}\left(X_{0}, 0\right), 0\right)\right) \wedge G^{-}\left(X^{-}\left(t, \tilde{b}\left(X_{0}, 0\right), 0\right)\right)\right)}{b^{-}\left(T_{2}, t, \tilde{b}\left(X_{0}, 0\right)\right)\left\|F^{-}\left(X^{-}\left(t, \tilde{b}\left(X_{0}, 0\right), 0\right)\right)\right\|^{2}} \mathrm{~d} t .
\end{aligned}
$$

$e$

Demonstração: A aplicação $t \mapsto X_{\varepsilon}^{-}$onde

$$
X_{\varepsilon}^{-}=\frac{\partial}{\partial \varepsilon} X^{-}\left(t, X_{0}, \varepsilon\right)
$$

é uma solução da equação variacional

$$
\dot{W}=D F^{-}\left[X^{-}\left(t, X_{0}, 0\right)\right] W+G^{-}\left(X^{-}\left(t, X_{0}, 0\right)\right), \quad W(0)=0 .
$$


De fato, como $X^{-}=X^{-}\left(t, X_{0}, \varepsilon\right)$ é solução de

$$
\dot{X}=F^{-}(X)+\varepsilon G^{-}(X), \quad X(0)=X_{0},
$$

segue da dependência diferenciável das soluções com respeito aos parâmetros e condições iniciais que uma solução de (4.64) é diferenciável com respeito ao parâmetro $\varepsilon$. Portanto, substituindo na equação (4.78) $X^{-}$e derivando com respeito a $\varepsilon$ obtemos

$$
\dot{X}_{\varepsilon}^{-}=D F^{-}\left[X^{-}\right] X_{\varepsilon}^{-}+G^{-}\left(X^{-}\right)+\varepsilon G_{\varepsilon}^{-}\left(X^{-}\right) X_{\varepsilon}^{-}, \quad X^{-}(0)_{\varepsilon}=\left(X_{0}\right)_{\varepsilon} .
$$

Tomando $\varepsilon=0$ obtemos a equação variacional (4.77).

Da mesma forma $t \mapsto X_{\varepsilon}^{+}$onde

$$
X_{\varepsilon}^{+}=\frac{\partial}{\partial \varepsilon} X^{+}\left(t, X_{1 \Sigma}, \varepsilon\right)
$$

é solução de

$$
\dot{W}=D F^{+}\left[X^{+}\left(t, X_{1 \Sigma}, 0\right)\right] W+G^{+}\left(X^{+}\left(t, X_{1 \Sigma}, 0\right)\right), \quad W(0)=0,
$$

e também, $t \mapsto X_{\varepsilon}^{-}$onde

$$
X_{\varepsilon}^{-}=\frac{\partial}{\partial \varepsilon} X^{-}\left(t, X_{2 \Sigma}, \varepsilon\right)
$$

é solução de

$$
\dot{W}=D F^{-}\left[X^{-}\left(t, X_{2 \Sigma}, 0\right)\right] W+G^{-}\left(X^{-}\left(t, X_{2 \Sigma}, 0\right)\right), \quad W(0)=0 .
$$

Pela Método da Variação de Parâmetros obtemos

$$
\left.X_{\varepsilon}^{-}\left(T_{1}, X_{0}, \varepsilon\right)\right|_{\varepsilon=0}=\Phi\left(0, T_{1}\right) \int_{0}^{T_{1}} \Phi^{-1}(0, s) G^{-}\left(X^{-}\left(s, X_{0}, 0\right)\right) \mathrm{d} s,
$$

onde $\Phi$ é a matriz fundamental de soluções.

Da mesma forma

$$
\left.X_{\varepsilon}^{+}\left(T_{2}, X_{1 \Sigma}, \varepsilon\right)\right|_{\varepsilon=0}=\Phi\left(T_{1}, T_{2}\right) \int_{T_{1}}^{T_{2}} \Phi^{-1}\left(T_{1}, s\right) G^{+}\left(X^{+}\left(s, X_{1 \Sigma}, 0\right)\right) \mathrm{d} s
$$

$$
\left.X_{\varepsilon}^{-}\left(T_{3}, X_{2 \Sigma}, \varepsilon\right)\right|_{\varepsilon=0}=\Phi\left(T_{2}, T_{3}\right) \int_{T_{2}}^{T_{3}} \Phi^{-1}\left(T_{2}, s\right) G^{-}\left(X^{-}\left(s, X_{2 \Sigma}, 0\right)\right) \mathrm{d} s .
$$

Similar a prova do Teorema 2.1.8 (Lema Variacional) da Seção 2.1 podemos escrever

$$
G^{-}\left(X^{-}\left(s, X_{0}, 0\right)\right)=c_{1}\left(s, X_{0}\right) F^{-}\left(X^{-}\left(s, X_{0}, 0\right)\right)+c_{2}\left(s, X_{0}\right)\left(F^{-}\right)^{\perp}\left(X\left(s, X_{0}, 0\right)\right),
$$

onde

$$
c_{1}\left(s, X_{0}\right)=\frac{1}{\left\|F^{-}\left(X\left(s, X_{0}, 0\right)\right)\right\|^{2}}\left\langle G^{-}\left(X^{-}\left(s, X_{0}, 0\right)\right), F^{-}\left(X^{-}\left(s, x_{0}, 0\right)\right)\right\rangle,
$$




$$
\begin{aligned}
c_{2}\left(s, X_{0}\right) & =\frac{1}{\left\|\left(F^{-}\right)^{\perp}\left(X^{-}\left(s, X_{0}, 0\right)\right)\right\|^{2}}\left\langle G^{-}\left(X^{-}\left(s, X_{0}, 0\right)\right),\left(F^{-}\right)^{\perp}\left(X^{-}\left(s, X_{0}, 0\right)\right)\right\rangle \\
& =\frac{1}{\left\|F^{-}\left(X^{-}\left(s, X_{0}, 0\right)\right)\right\|^{2}} F^{-}\left(X^{-}\left(s, X_{0}, 0\right)\right) \wedge\left(G^{-}\right)\left(X^{-}\left(s, X_{0}, 0\right)\right) .
\end{aligned}
$$

Pela Teorema 4.3.4, temos

$$
\Phi(0, s)=\left(\begin{array}{cc}
1 & a^{-}\left(0, s, X_{0}\right) \\
0 & b^{-}\left(0, s, X_{0}\right)
\end{array}\right)
$$

Assim,

$$
\Phi^{-1}(0, s) G^{-}\left(X^{-}\left(s, X_{0}, 0\right)\right)=\frac{1}{b^{-}\left(0, s, X_{0}\right)}\left(\begin{array}{cc}
b^{-}\left(0, s, X_{0}\right) & -a^{-}\left(0, s, X_{0}\right) \\
0 & 1
\end{array}\right)\left(\begin{array}{l}
c_{1}\left(s, X_{0}\right) \\
c_{2}\left(s, X_{0}\right)
\end{array}\right) .
$$

Agora, substituindo $\Phi^{-1}(0, s) G^{-}\left(X^{-}\left(s, X_{0}, 0\right)\right)$, na equação (4.79) obtemos (4.74). De forma similar obtemos as outras expressões dadas em (4.75) e (4.76).

\subsubsection{Continuação de Órbitas Periódicas}

Considere a aplicação de Poincaré do sistema (4.64) dada por

$$
\begin{aligned}
\Pi: \widetilde{\Sigma} \times\left[0, \varepsilon_{0}\right) & \longrightarrow \Sigma \\
(X, \varepsilon) & \longmapsto \Pi(X, \varepsilon),
\end{aligned}
$$

onde $\widetilde{\Sigma} \subseteq \Sigma$ é transversal às órbitas periódicas do sistema (Seção de Poincaré). Tome uma função (parametrização local de $\widetilde{\Sigma}$ )

$$
\begin{gathered}
\sigma: \mathbb{R} \longrightarrow \widetilde{\Sigma} \\
a \longmapsto \sigma(a)
\end{gathered}
$$

tal que $\sigma(0)=X_{0}$ e para cada $a \in \mathbb{R}$ o vetor $\dot{\sigma}(a)$ é um vetor não nulo tangente à $\widetilde{\Sigma}$ em $\sigma(a)$.

Definimos a função de separação por

$$
\delta(X, \varepsilon)=\sigma^{-1}(\Pi(X, \varepsilon))-\sigma^{-1}(X)
$$

ou em coordenadas locais por

$$
\delta(a, \varepsilon)=\sigma^{-1}(\Pi(\sigma(a), \varepsilon))-a .
$$

Agora, como o campo $F$ dado no sistema (4.63) tem um anel $\mathcal{A}$ de órbitas periódicas, então $\widetilde{\Sigma}$ (seção de Poincaré) é transversal a estas órbitas em $\sigma$. Assim $\delta(X, 0) \equiv 0$ para todo $X \in \mathcal{A}$, pois

$$
\delta(X, 0)=\sigma^{-1}(\Pi(X, 0))-\sigma^{-1}(X)=\sigma^{-1}(X)-\sigma^{-1}(X)=0 .
$$


Portanto, não podemos aplicar o Teorema da Função Implícita para a função $\delta$ diretamente. Como nas seções anteriores, precisamos definir uma função de bifurcação. Para definir a função de bifurcação, devemos calcular a derivada de $\delta$ com respeito a $\varepsilon$ primeiramente.

A aplicação de Poincaré é assim definida

$$
\Pi(X, \varepsilon)=X^{-}\left(\tau_{3}(X, \varepsilon), X^{+}\left(\tau_{2}(X, \varepsilon), X^{-}\left(\tau_{1}(X, \varepsilon), X, \varepsilon\right), \varepsilon\right), \varepsilon\right) .
$$

Denote

$$
\begin{aligned}
& X^{-}\left(\tau_{3}\right)=X^{-}\left(\tau_{3}(X, \varepsilon), X^{+}\left(\tau_{2}(X, \varepsilon), X^{-}\left(\tau_{1}(X, \varepsilon), X, \varepsilon\right), \varepsilon\right), \varepsilon\right) \\
& X^{+}\left(\tau_{2}\right)=X^{+}\left(\tau_{2}(X, \varepsilon), X^{-}\left(\tau_{1}(X, \varepsilon), X, \varepsilon\right), \varepsilon\right) \\
& X^{-}\left(\tau_{1}\right)=X^{-}\left(\tau_{1}(X, \varepsilon), X, \varepsilon\right) .
\end{aligned}
$$

Derivando $\Pi$ com respeito a $\varepsilon$ obtemos

$$
\begin{aligned}
\frac{\partial \Pi}{\partial \varepsilon}(X, \varepsilon) & =\Pi_{\varepsilon}(X, \varepsilon)=D_{1} X^{-}\left(\tau_{3}\right) \frac{\partial \tau_{3}}{\partial \varepsilon}(X, \varepsilon)+D_{2} X^{-}\left(\tau_{3}\right)\left\{D_{1} X^{+}\left(\tau_{2}\right) \frac{\partial \tau_{2}}{\partial \varepsilon}(X, \varepsilon)\right. \\
& +D_{2} X^{+}\left(\tau_{2}\right)\left[D_{1} X^{-}\left(\tau_{1}\right) \frac{\partial \tau_{1}}{\partial \varepsilon}(X, \varepsilon)+D_{2} X^{-}\left(\tau_{1}\right)+D_{3} X^{-}\left(\tau_{1}\right)\right] \\
& \left.+D_{3} X^{+}\left(\tau_{2}\right)\right\}+D_{3} X^{-}\left(\tau_{3}\right) .
\end{aligned}
$$

Temos que

$$
\begin{aligned}
& D_{1} X^{-}\left(\tau_{3}\right)=F^{-}\left(X^{-}\left(\tau_{3}\right)\right)+\varepsilon G^{-}\left(X^{-}\left(\tau_{3}\right)\right), \\
& D_{1} X^{+}\left(\tau_{2}\right)=F^{+}\left(X^{+}\left(\tau_{2}\right)\right)+\varepsilon G^{+}\left(X^{+}\left(\tau_{2}\right)\right), \\
& D_{1} X^{-}\left(\tau_{1}\right)=F^{-}\left(X^{-}\left(\tau_{1}\right)\right)+\varepsilon G^{-}\left(X^{-}\left(\tau_{1}\right)\right) .
\end{aligned}
$$

Portanto,

$$
\begin{aligned}
\Pi_{\varepsilon}(X, \varepsilon) & =\left(F^{-}\left(X^{-}\left(\tau_{3}\right)\right)+\varepsilon G^{-}\left(X^{-}\left(\tau_{3}\right)\right)\right) \frac{\partial \tau_{3}}{\partial \varepsilon}(X, \varepsilon) \\
& +D_{2} X^{-}\left(\tau_{3}\right)\left\{\left(F^{+}\left(X^{+}\left(\tau_{2}\right)\right)+\varepsilon G^{+}\left(X^{+}\left(\tau_{2}\right)\right)\right) \frac{\partial \tau_{2}}{\partial \varepsilon}(X, \varepsilon)\right. \\
& +D_{2} X^{+}\left(\tau_{2}\right)\left[\left(F^{-}\left(X^{-}\left(\tau_{1}\right)\right)+\varepsilon G^{-}\left(X^{-}\left(\tau_{1}\right)\right)\right) \frac{\partial \tau_{1}}{\partial \varepsilon}(X, \varepsilon)\right. \\
& \left.\left.+D_{2} X^{-}\left(\tau_{1}\right)+D_{3} X^{-}\left(\tau_{1}\right)\right]+D_{3} X^{+}\left(\tau_{2}\right)\right\}+D_{3} X^{-}\left(\tau_{3}\right) .
\end{aligned}
$$

O termo $D_{2} X^{+}\left(\tau_{2}\right)$ pode ser obtido no Teorema 4.3.2 e o termo $D_{2} X^{-}\left(\tau_{3}\right)$ no Teorema 4.3.3. Agora, $D_{3} X^{-}\left(\tau_{1}\right), D_{3} X^{-}\left(\tau_{2}\right)$ e $D_{3} X^{-}\left(\tau_{3}\right)$ estão expressos no Teorema 4.3.5. Resta portanto, obter

$$
\frac{\partial \tau_{1}}{\partial \varepsilon}(X, \varepsilon), \quad \frac{\partial \tau_{2}}{\partial \varepsilon}(X, \varepsilon) \quad \text { e } \quad \frac{\partial \tau_{3}}{\partial \varepsilon}(X, \varepsilon) .
$$

Calculemos

$$
\frac{\partial \tau_{1}}{\partial \varepsilon}(X, \varepsilon) \quad \text { e } \frac{\partial \tau_{2}}{\partial \varepsilon}(X, \varepsilon) .
$$

Como $X^{-}\left(\tau_{1}\right)=X^{-}\left(\tau_{1}(X, \varepsilon), X, \varepsilon\right) \in \Sigma$, então

$$
\mathcal{H}\left(X^{-}\left(\tau_{1}(X, \varepsilon), X, \varepsilon\right)\right)=0
$$


Derivando (4.82) com respeito a $\varepsilon$ obtemos

$$
D \mathcal{H}\left(X^{-}\left(\tau_{1}\right)\right)\left[D_{1} X^{-}\left(\tau_{1}\right) \frac{\partial \tau_{1}}{\partial \varepsilon}(X, \varepsilon)+D_{3} X^{-}\left(\tau_{1}\right)\right]=0 .
$$

Então,

$$
\frac{\partial \tau_{1}}{\partial \varepsilon}(X, \varepsilon)=-\frac{N^{T}\left(X^{-}\left(\tau_{1}\right)\right) D_{3} X^{-}\left(\tau_{1}\right)}{N^{T}\left(X^{-}\left(\tau_{1}\right)\right) \cdot\left[F^{-}\left(X^{-}\left(\tau_{1}\right)\right)+\varepsilon G^{-}\left(X^{-}\left(\tau_{1}\right)\right)\right]} .
$$

Veja que $X^{+}\left(\tau_{2}\right)=X^{+}\left(\tau_{2}(X, \varepsilon), X^{-}\left(\tau_{1}(X, \varepsilon), X, \varepsilon\right), \varepsilon\right) \in \Sigma$, então

$$
\mathcal{H}\left(X^{+}\left(\tau_{2}(X, \varepsilon), X^{-}\left(\tau_{1}(X, \varepsilon), X, \varepsilon\right), \varepsilon\right)\right)=0
$$

Derivando (4.83) com respeito a $\varepsilon$ obtemos

$$
D \mathcal{H}\left(X^{-}\left(\tau_{2}\right)\right)\left\{D_{1} X^{+}\left(\tau_{2}\right) \frac{\partial \tau_{2}}{\partial \varepsilon}(X, \varepsilon)+D_{2} X^{+}\left(\tau_{2}\right)\left[D_{1} X^{-}\left(\tau_{1}\right) \frac{\partial \tau_{1}}{\partial \varepsilon}(X, \varepsilon)+D_{3} X^{-}\left(\tau_{1}\right)\right]+D_{3} X^{+}\left(\tau_{2}\right)\right\}=0 .
$$

Então,

$$
\begin{aligned}
\frac{\partial \tau_{2}}{\partial \varepsilon}(X, \varepsilon) & =-\frac{N^{T}\left(X^{+}\left(\tau_{2}\right)\right) D_{2} X^{+}\left(\tau_{2}\right)\left[F^{-}\left(X^{-}\left(\tau_{1}\right)\right)+\varepsilon G^{-}\left(X^{-}\left(\tau_{1}\right)\right)\right] \frac{\partial \tau_{1}}{\partial \varepsilon}(X, \varepsilon)}{N^{T}\left(X^{+}\left(\tau_{2}\right)\right) \cdot\left[F^{+}\left(X^{+}\left(\tau_{2}\right)\right)+\varepsilon G^{+}\left(X^{+}\left(\tau_{2}\right)\right)\right]} \\
& -\frac{N^{T}\left(X^{+}\left(\tau_{2}\right)\right) D_{2} X^{+}\left(\tau_{2}\right) D_{3} X^{-}\left(\tau_{1}\right)}{N^{T}\left(X^{+}\left(\tau_{2}\right)\right) \cdot\left[F^{+}\left(X^{+}\left(\tau_{2}\right)\right)+\varepsilon G^{+}\left(X^{+}\left(\tau_{2}\right)\right)\right]} \\
& -\frac{N^{T}\left(X^{+}\left(\tau_{2}\right)\right) D_{3} X^{+}\left(\tau_{2}\right)}{N^{T}\left(X^{+}\left(\tau_{2}\right)\right) \cdot\left[F^{+}\left(X^{+}\left(\tau_{2}\right)\right)+\varepsilon G^{+}\left(X^{+}\left(\tau_{2}\right)\right)\right]} .
\end{aligned}
$$

Denotemos

$$
\begin{gathered}
A(s, X, \varepsilon):=D_{2} X^{-}\left(s, X^{+}\left(\tau_{2}(X, \varepsilon), X^{-}\left(\tau_{1}(X, \varepsilon), X, \varepsilon\right), \varepsilon\right), \varepsilon\right), \\
B(s, X, \varepsilon):=D_{2} X^{+}\left(s, X^{-}\left(\tau_{1}(X, \varepsilon), X, \varepsilon\right), \varepsilon\right), \\
C(s, X, \varepsilon):=D_{2} X^{-}(s, X, \varepsilon),
\end{gathered}
$$

e

$$
\begin{gathered}
\tilde{a}(X, \varepsilon):=X^{-}\left(\tau_{1}(X, \varepsilon), X, \varepsilon\right), \\
\tilde{b}(X, \varepsilon):=X^{+}\left(\tau_{2}(X, \varepsilon), X^{-}\left(\tau_{1}(X, \varepsilon), X, \varepsilon\right), \varepsilon\right),
\end{gathered}
$$

com

$$
\begin{gathered}
\tilde{a}\left(X_{0}, 0\right)=X^{-}\left(T_{1}, X_{0}, 0\right)=X_{1 \Sigma} \\
\tilde{b}\left(X_{0}, 0\right)=X^{+}\left(T_{2}, X^{-}\left(T_{1}, X_{0}, 0\right), 0\right)=X_{2 \Sigma} .
\end{gathered}
$$

Assim,

$$
\begin{aligned}
\left.\Pi_{\varepsilon}(X, \varepsilon)\right|_{\varepsilon=0, X=X_{0}} & =F^{-}\left(X^{-}\left(T_{3}, X_{2 \Sigma}, 0\right)\right) \frac{\partial \tau_{3}}{\partial \varepsilon}\left(X_{0}, 0\right) \\
& +A\left(T_{3}, X_{0}, 0\right)\left\{F^{+}\left(X_{2 \Sigma}\right) \frac{\partial \tau_{2}}{\partial \varepsilon}\left(X_{0}, 0\right)\right. \\
& +B\left(T_{2}, X_{0}, 0\right)\left[\left(F^{-}\left(X_{1 \Sigma}\right)\right) \frac{\partial \tau_{1}}{\partial \varepsilon}\left(X_{0}, 0\right)\right. \\
& \left.\left.+C\left(T_{1}, X_{0}, 0\right)+D_{3} \tilde{a}\left(X_{0}, 0\right)\right]+D_{3} \tilde{b}\left(X_{0}, 0\right)\right\} \\
& +D_{3} X^{-}\left(T_{3}, X_{2 \Sigma}, 0\right) .
\end{aligned}
$$


Como $X^{-}\left(T_{3}, X_{2 \Sigma}, 0\right)=X_{0}$ assim,

$$
\begin{aligned}
\left.\Pi_{\varepsilon}(X, \varepsilon)\right|_{\varepsilon=0, X=X_{0}} & =F^{-}\left(X_{0}\right) \frac{\partial \tau_{3}}{\partial \varepsilon}\left(X_{0}, 0\right) \\
& +A\left(T_{3}, X_{0}, 0\right)\left(F^{+}\left(X_{2 \Sigma}\right) \frac{\partial \tau_{2}}{\partial \varepsilon}\left(X_{0}, 0\right)\right. \\
& +A\left(T_{3}, X_{0}, 0\right) B\left(T_{2}, X_{0}, 0\right) F^{-}\left(X_{1 \Sigma}\right) \frac{\partial \tau_{1}}{\partial \varepsilon}\left(X_{0}, 0\right) \\
& +A\left(T_{3}, X_{0}, 0\right) B\left(T_{2}, X_{0}, 0\right) C\left(T_{1}, X_{0}, 0\right) \\
& +A\left(T_{3}, X_{0}, 0\right) B\left(T_{2}, X_{0}, 0\right) D_{3} \tilde{a}\left(X_{0}, 0\right) \\
& +A\left(T_{3}, X_{0}, 0\right) D_{3} \tilde{b}\left(X_{0}, 0\right)+D_{3} X^{-}\left(T_{3}, X_{2 \Sigma}, 0\right) .
\end{aligned}
$$

Temos que as derivadas de $\tilde{a}(X, \varepsilon)$ e $\tilde{b}(X, \varepsilon)$ com respeito a $\varepsilon$ são dadas por

$$
\begin{aligned}
& D_{3} \tilde{a}(X, \varepsilon)=D_{1} X^{-}\left(\tau_{1}\right) \frac{\partial \tau_{1}}{\partial \varepsilon}(X, \varepsilon)+D_{3} X^{-}\left(\tau_{1}\right), \\
D_{3} \tilde{b}(X, \varepsilon) & =D_{1} X^{+}\left(\tau_{2}\right) \frac{\partial \tau_{2}}{\partial \varepsilon}(X, \varepsilon)+D_{2} X^{+}\left(\tau_{2}\right)\left[D_{1} X^{-}\left(\tau_{1}\right) \frac{\partial \tau_{1}}{\partial \varepsilon}(X, \varepsilon)\right. \\
& \left.+D_{3} X^{-}\left(\tau_{1}\right)\right]+D_{3} X^{+}\left(\tau_{2}\right) .
\end{aligned}
$$

Avaliando em $X=X_{0}$ e $\varepsilon=0$ obtemos

$$
\begin{aligned}
& D_{3} \tilde{a}\left(X_{0}, 0\right)=F^{-}\left(X_{1 \Sigma}\right) \frac{\partial \tau_{1}}{\partial \varepsilon}\left(X_{0}, 0\right)+D_{3} X^{-}\left(T_{1}, X_{0}, 0\right) \\
D_{3} \tilde{b}\left(X_{0}, 0\right) & =F^{+}\left(X_{2 \Sigma}\right) \frac{\partial \tau_{2}}{\partial \varepsilon}\left(X_{0}, 0\right)+D_{2} X^{+}\left(T_{2}, X_{1 \Sigma}, 0\right)\left[F^{-}\left(X_{1 \Sigma}\right) \frac{\partial \tau_{1}}{\partial \varepsilon}\left(X_{0}, 0\right)\right. \\
& \left.+D_{3} X^{-}\left(T_{1}, X_{0}, 0\right)\right]+D_{3} X^{+}\left(T_{2}, X^{-}\left(T_{1}, X_{0}, 0\right), 0\right) .
\end{aligned}
$$

Para obter $A\left(T_{3}, X_{0}, 0\right), B\left(T_{2}, X_{0}, 0\right)$ e $C\left(T_{1}, X_{0}, 0\right)$ podemos usar o Teorema 4.3.4, obtendo

$$
\begin{aligned}
& A\left(T_{3}, X_{0}, 0\right)=\Phi\left(T_{2}, T_{3}\right) \cdot A\left(T_{2}, X_{0}, 0\right)=\left(\begin{array}{ll}
1 & a^{-}\left(T_{2}, T_{3}, X_{2 \Sigma}\right) \\
0 & b^{-}\left(T_{2}, T_{3}, X_{2 \Sigma}\right)
\end{array}\right) \cdot A\left(T_{2}, X_{0}, 0\right), \\
& B\left(T_{2}, X_{0}, 0\right)=\Phi\left(T_{1}, T_{2}\right) \cdot B\left(T_{1}, X_{0}, 0\right)=\left(\begin{array}{ll}
1 & a^{-}\left(T_{1}, T_{2}, X_{1 \Sigma}\right) \\
0 & b^{-}\left(T_{1}, T_{2}, X_{1 \Sigma}\right)
\end{array}\right) \cdot B\left(T_{1}, X_{0}, 0\right) .
\end{aligned}
$$

e

$$
C\left(T_{1}, X_{0}, 0\right)=\Phi\left(0, T_{1}\right) \cdot C\left(0, X_{0}, 0\right)=\left(\begin{array}{cc}
1 & a^{-}\left(0, T_{1}, X_{0}\right) \\
0 & b^{-}\left(0, T_{1}, X_{0}\right)
\end{array}\right) \cdot C\left(0, X_{0}, 0\right) .
$$

Além disso, $A\left(T_{2}, X_{0}, 0\right), B\left(T_{1}, X_{0}, 0\right)$ e $C\left(0, X_{0}, 0\right)$ podem ser obtidas nas equações (4.65) e (4.68).

Agora, pela fórmula de $\delta$, temos

$$
\delta(X, \varepsilon)+\sigma^{-1}(X)=\sigma^{-1}(\pi(X, \varepsilon))
$$

Então,

$$
\Pi(X, \varepsilon)=\sigma\left(\delta(X, \varepsilon)+\sigma^{-1}(X)\right)
$$


Derivando com respeito a $\varepsilon$ obtemos

$$
\Pi_{\varepsilon}(X, \varepsilon)=\dot{\sigma}\left(\delta(X, \varepsilon)+\sigma^{-1}(X)\right) \cdot\left(\delta_{\varepsilon}(X, \varepsilon)+\sigma_{\varepsilon}^{-1}(X)\right) .
$$

Como $\delta\left(X_{0}, 0\right)=0, \sigma^{-1}\left(X_{0}\right)=0$ e $\sigma_{\varepsilon}^{-1}=0$, obtém-se

$$
\Pi_{\varepsilon}\left(X_{0}, 0\right)=\dot{\sigma}(0) \cdot \delta_{\varepsilon}\left(X_{0}, 0\right)
$$

Tomando o produto interno com $\left(F^{-}\right)^{\perp}\left(X_{0}\right)$, obtemos

$$
\delta_{\varepsilon}\left(X_{0}, 0\right)\left\langle\dot{\sigma}(0),\left(F^{-}\right)^{\perp}\left(X_{0}\right)\right\rangle=\left\langle\Pi_{\varepsilon}\left(X_{0}, 0\right),\left(F^{-}\right)^{\perp}\left(X_{0}\right)\right\rangle .
$$

O segundo membro desta expressão não possui o termo $F^{-}\left(X_{0}\right) \frac{\partial \tau_{3}}{\partial \varepsilon}\left(X_{0}, 0\right)$, logo todos os termos de (4.84) podem ser obtidos. A equação (4.85) sugere a definição a seguir.

Definição 4.3.1. Da equação (4.85), obtemos $\delta_{\varepsilon}$, e definimos a função de bifurcação $\mathcal{B}(a)$, da seguinte forma

$$
\mathcal{B}\left(X_{0}\right)=\frac{\left\langle\Pi_{\varepsilon}\left(X_{0}, 0\right),\left(F^{-}\right)^{\perp}\left(X_{0}\right)\right\rangle}{\left\langle\dot{\sigma}(0),\left(F^{-}\right)^{\perp}\left(X_{0}\right)\right\rangle} .
$$

Veja que $\left\langle\dot{\sigma}(0),\left(F^{-}\right)^{\perp}\left(X_{0}\right)\right\rangle \neq 0$, pois, as órbitas periódicas interceptam $\widetilde{\Sigma}$ transversalmente.

Teorema 4.3.6. Considere o sistema perturbado (4.64) com solução $X(t, Y, \varepsilon)$ onde $X(0)=Y$ tal que o sistema não perturbado (4.63) tenha um anel $\mathcal{A}$ de órbitas periódicas com seção de Poincaré $\widetilde{\Sigma}$. Se existe um ponto $X_{0} \in \widetilde{\Sigma}$ tal que a função de separação $\delta$ satisfaz $\delta_{\varepsilon}\left(X_{0}, 0\right)=0$ e $\delta_{Y \varepsilon}\left(X_{0}, 0\right) \neq 0$, então, existe uma órbita periódica $\Gamma$ do sistema (4.63) transversal a $\widetilde{\Sigma}$ em $X_{0} e$ uma família contínua $\Gamma^{\varepsilon}$ de órbitas periódicas do sistema (4.64) bifurcando de $\Gamma$.

Demonstração: Temos que $\delta(Y, 0) \equiv 0$ para todo $Y \in \mathcal{A}$ e a expansão de Taylor da função $\delta$ em (4.80) com respeito a $\varepsilon$ em torno de $\varepsilon=0$ é dada por

$$
\delta(Y, \varepsilon)=\varepsilon\left(\delta_{\varepsilon}(Y, 0)+O(Y, \varepsilon)\right)=\varepsilon \Delta(Y, \varepsilon)
$$

sendo

$$
\delta_{\varepsilon}(Y, 0)=\left.\frac{\partial}{\partial \varepsilon} \delta(Y, \varepsilon)\right|_{\varepsilon=0} .
$$

Segue das hipóteses que

$$
\Delta\left(X_{0}, 0\right)=\delta_{\varepsilon}\left(X_{0}, 0\right)=0 \quad \text { e } \quad \Delta_{Y}\left(X_{0}, 0\right)=\delta_{Y \varepsilon}\left(X_{0}, 0\right) \neq 0
$$

onde

$$
\Delta_{Y}(Y, 0)=\left.\frac{\partial}{\partial Y} \Delta(Y, \varepsilon)\right|_{\varepsilon=0} .
$$

Pelo Teorema da Função Implícita aplicado a função $\Delta$, existe intervalo aberto $I \subset\left[0, \varepsilon_{0}\right)$ e uma única função $\varepsilon \mapsto \phi(\varepsilon)$, com $\phi(\varepsilon) \in \widetilde{\Sigma}$ em $\mathbb{R}^{2}$ tal que

$$
\Delta(\phi(\varepsilon), \varepsilon)=0 \quad \text { e } \quad \phi(0)=X_{0} .
$$


Logo a equação $\delta(Y, \varepsilon)=0$ tem uma solução implícita $Y=\phi(\varepsilon)$. Portanto, existe uma única solução $\Gamma$ encontrando $\widetilde{\Sigma}$ transversalmente em $X_{0}=\phi(0)$ e para $\varepsilon>0$ suficientemente pequeno em $I \subset\left[0, \varepsilon_{0}\right)$ a família de órbitas periódicas $\Gamma^{\varepsilon}$ bifurca de $\Gamma$, isto é, $\Gamma^{\varepsilon}$ tende a $\Gamma$ quando $\varepsilon$ tende a 0 .

Observação 4.3.2. Os resultados acima são imediatamente adaptados no caso em que $F^{-}(X)=$ $F^{+}(X)$ no sistema (4.64). No artigo [1] os autores consideram o caso em que $G^{-}(X)=G^{+}(X)$ no sistema (4.64) na qual adaptamos nesta seção para o sistema (4.64) o mais geral possível. 


\section{Capítulo 5}

\section{Conclusões}

Nesta tese estudamos os sistemas de equações diferenciais ordinárias suaves por partes com duas zonas no plano. Mostramos que na classe de sistemas lineares homogêneos com coeficientes constantes a origem é globalmente assintoticamente estável quando as matrizes que definem o sistema são Hurwitz, o conjunto de separação $\Sigma$ é uma reta que contém o ponto de equilíbrio (que é a origem) e os pontos sobre $\Sigma$ (exceto a origem) são pontos de costura. Neste contexto, mostramos que simplesmente trocando $\Sigma$ por uma curva poligonal podem existir matrizes Hurwitz tal que a origem é instável. Concluimos que a hipótese sobre o tipo do conjunto de separação $\Sigma$ é essencial no comportamento qualitativo do sistema.

Em algumas classes de sistemas por partes com duas zonas no plano, sob algumas hipóteses em cada caso, obtivemos as funções de Melnikov, com o objetivo principal de determinar quantos ciclos limites (de costura) bifurcam de um conjunto de órbitas periódicas (em geral dadas por curvas suaves por partes). Este problema é dado em geral por uma perturbação suave por partes de um sistema suave por partes.

Uma outra ferramenta para o estudo de sistemas por partes é o método de regularização. Como o próprio nome diz, o método consiste em tornar regular (suave) um problema descontínuo como o dos sistemas descontínuos. A vantagem de tal método é que a teoria suave pode ser aplicada sem receios. Um questão que surge naturalmente é a de como se relacionam o problema descontínuo e o problema regularizado. Sob este ponto de vista, considerando um centro linear perturbado por partes e um centro Hamiltoniano perturbado por partes, mostramos que as funções de Melnikov em ambos os casos podem ser obtidas como um processo limite das funções de Melnikov dos sistemas regularizados. Portanto, usando o método de regularização, damos uma prova que justifica o uso de funções de Melnikov diretamente do problema descontínuo original. Uma proposta de trabalho futuro é a de obter tal relação para outros casos. Outra direção de pesquisa é baseada no recente artigo [17, onde a equivalência entre as funções Melnikov e a teoria da média para sistemas suaves foi estabelecida. 


\section{Bibliografia}

[1] Z. Afsharnezhad, M.K. Amaleh, Continuation of the periodic orbits for the differential equation with discontinuous right hand side, J. Dynam. Differential Equations, 23 (2011), $71-92$.

[2] A. Andronov, A. Vitt, S. Khaikin, Theory of Oscillators, Pergamon Press Inc., UK, 1966.

[3] C. Arzelá, Sulla integrazione per serie, Atti Acc. Lincei Rend., 1 (1885), 532-537, 596-599.

[4] D.C. Braga, A.F. Fonseca, L.F. Mello, The matching of two stable sewing linear systems in the plane, J. Math. Anal. Appl., 2 (2015), 1142-1156.

[5] D.C. Braga, A.F. Fonseca, L.F. Mello, Melnikov functions and limit cycles in piecewise smooth perturbations of a linear center using regularization method, submetido, 2016.

[6] D.C. Braga, L.F. Mello, Limit cycles in a family of discontinuous piecewise linear differential systems with two zones in the plane, Nonlinear Dynam., 73 (2013), 1283-1288.

[7] D.C. Braga, L.F. Mello, More than three limit cycles in discontinuous piecewise linear differential systems with two zones in the plane, Internat. J. Bifur. Chaos Appl. Sci. Engrg., 24 (2014), 1450056 (10 pages).

[8] D.C. Braga, L.F. Mello, Arbitrary number of limit cycles for a class of planar discontinuous piecewise linear differential systems with two zones, Electron. J. Differential Equation, 2015 (2015), 1-12.

[9] B. Brogliato, Nonsmooth Impact Mechanics, Springer-Verlag, London 1996.

[10] V. Carmona, E. Freire, E. Ponce, F. Torres, The continuous matching of two stable linear systems can be unstable, Discrete Contin. Dyn. Syst., 16 (2006), 689-703.

[11] C. Chicone, Ordinary Differential Equations With Applications, Springer-Verlag, New York, 1999. 
[12] M. di Bernardo, C.J. Budd, A.R. Champneys, P. Kowalczyk, Piecewise-Smooth Dynamical Systems: Theory and Applications, Springer-Verlag, London, 2008.

[13] S.P. Diliberto, On systems of ordinary differential equations, in Contributions to the Theory of Nonlinear Oscillations, Ann. of Math. Stud., 20 (1950), 1-38.

[14] A.F. Filippov, Differential equations with discontinuous righthand sides, Kluwer, Dordrecht, 1988.

[15] E. Freire, E. Ponce, F. Rodrigo, F. Torres, Bifurcation sets of continuous piecewise linear systems with two zones, Internat. J. Bifur. Chaos Appl. Sci. Engrg., 8 (1998), 20732097.

[16] E. Freire, E. Ponce, F. Torres, Canonical discontinuous planar piecewise linear systems, SIAM J. Appl. Dyn. Syst., 11 (2012), 181-211.

[17] M. Han, V.G. Romanovski, X. Zhang, Equivalence of the Melnikov function method and the averaging method, Qual. Theory Dyn. Syst., DOI 10.1007/s12346-015-0179-3, preprint, 2015.

[18] M.W. Hirsch, S. Smale, R.L. Devaney, Differential Equations, Dynamical Systems, and an Introduction to Chaos, Elsevier, USA, 2004.

[19] Y. Iwatani, S. Hara, Stability tests and stabilization for piecewise linear systems based on poles and zeros of subsystems, Automatica, 42 (2006), 1685-1695.

[20] P. KUKUKA, Jumps of the fundamental matriz solutions in discontinuous and applications, Nonlinear Anal., 66 (11) (2007), 2529-2546.

[21] M. Kunze, Non-smooth Dynamical Systems, Springer-Verlag, Berlin, 2000.

[22] Y.A. Kuznetsov, S. Rinaldi, A. Grangnani, One parameter bifurcations in planar Filippov systems, Internat. J. Bifur. Chaos Appl. Sci. Engrg., 13 (8) (2003), 2157-2188.

[23] R.I. Leine, D.H. Van Campen, B.L. Van de VRande, Bifurcations in nonlinear discontinuous systems, Nonlinear Dyn., 23 (2) (2000), 105-164.

[24] X. Liu, M. Han, Bifurcation of limit cycles by perturbing piecewise Hamiltonian systems, Internat. J. Bifur. Chaos Appl. Sci. Engrg., 20 (2010), 1379-1390.

[25] J. Llibre, E. Ponce, Piecewise linear feedback systems with arbitrary number of limit cycles, Internat. J. Bifur. Chaos Appl. Sci. Engrg., 13 (2003), 895-904. 
[26] J. Llibre, E. Ponce, Three nested limit cycles in discontinuous piecewise linear differential systems with two zones, Dyn. Contin. Discrete Impuls. Syst. Ser. B Appl. Algorithms, 19 (2012), 325-335.

[27] J. Llibre, E. Ponce, X. Zhang, Existence of piecewise linear differential systems with exactly $n$ limit cycles for all $n \in \mathbb{N}$, Nonlinear Anal., 54 (2003), 977-994.

[28] J. Llibre, M.A. Teixeira, J. Torregrosa, Lower bounds for the maximum number of limit cycles of discontinuous piecewise linear differential systems with a straight line of separation, Internat. J. Bifur. Chaos Appl. Sci. Engrg., 23 (2013), 1350066.

[29] J. Llibre, A.C. Mereu, Limit cycles for discontinuous generalized Liénard polynomial differential equations, Electron. J. Differential Equations, vol. 2013 (2013), 1-8.

[30] A.L.F. Machado, Estabilidade Estrutural e Bifurcações de Campos Vetoriais Descontínuos, Tese, IME-USP, 2000.

[31] A.L. Maciel, Bifurcações de Campos Vetoriais Descontínuos, Tese, IME-USP, 2009.

[32] R.M. Martins, A.C. Mereu, Limit cycles in discontinuous classical Liénard equations, Nonlinear Anal. Real World Appl., 20 (2014), 67-73.

[33] J.C. Medrado, J. Torregrosa, Uniqueness of limit cycles for sewing piecewise linear systems, J. Math. Anal. Appl., 431 (2015), 529-544.

[34] C.P. Niculescu, F. Popovici, The monotone convergence theorem for the Riemann integral, An. Univ. Craiova Ser. Mat. Inform., 38 (2011), 55-58.

[35] W. Rudin, Principles of Mathematical Analysis, third edition, McGraw-Hill Book Company, Singapore, 1976.

[36] J. Sotomayor, Equações Diferenciais Ordinárias, Textos Universitários do IME-USP, Livraria da Física, São Paulo, 2012.

[37] J. Sotomayor, A.L.F. Machado, Structurally stable discontinuous vector fields in the plane, Qual. Theory Dyn. Syst., 3 (2002), 227-250.

[38] J. Sotomayor, M.A. Teixeira, Regularization of discontinuous vector fields, International Conference on Differential Equations (Lisboa, 1995), World Sci. Publ., River Edge, NJ, (1998), 207-223.

[39] G. Tigan, A. Astolfi, A note on a piecewise-linear Duffing-type system, Internat. J. Bifur. Chaos Appl. Sci. Engrg., 17 (2007), 4425-4429. 
[40] A. TONNELIER, On the number of limit cycles in piecewise linear Liénard systems, Internat. J. Bifur. Chaos Appl. Sci. Engrg., 15 (2005), 1417-1422. 